\title{
Physics perspectives of heavy-ion collisions at very high energy
}

\author{
Ning-bo Chang, ${ }^{1}$ Shanshan Cao, ${ }^{2}$ Bao-yi Chen, ${ }^{3}$ Shi-yong Chen, ${ }^{1}$ Zhen-yu Chen, ${ }^{3}$ Heng-Tong Ding, ${ }^{1}$ Min He, ${ }^{4}$ \\ Zhi-quan Liu, ${ }^{1}$ Long-gang Pang, ${ }^{1}$ Guang-you Qin, ${ }^{1}$ Ralf Rapp,${ }^{5}$ Björn Schenke, ${ }^{6}$ Chun Shen, ${ }^{7}$ Huichao Song, ${ }^{8}$ Hao-jie \\ $\mathrm{Xu},{ }^{9}$ Qun Wang, ${ }^{9}$ Xin-Nian Wang, ${ }^{1,2}$ Ben-wei Zhang, ${ }^{1}$ Han-zhong Zhang, ${ }^{1}$ Xiangrong Zhu, ${ }^{8}$ and Peng-fei Zhuang ${ }^{3}$ \\ ${ }^{1}$ Key Laboratory of Quark and Lepton Physics (MOE) and Institute of Particle Physics, \\ Central China Normal University, Wuhan 430079, China \\ ${ }^{2}$ Nuclear Science Division MS70R0319, Lawrence Berkeley National Laboratory, Berkeley, CA 94720 \\ ${ }^{3}$ Physics Department, Tsinghua University, Beijing 100084, China \\ ${ }^{4}$ Department of Applied Physics, Nanjing University of Science and Technology, Nanjing 210094, China \\ ${ }^{5}$ Cyclotron Institute and Department of Physics and Astronomy, \\ Texas A 63 M University, College Station, TX 77843, USA \\ ${ }^{6}$ Physics Department, Brookhaven National Laboratory, Upton, NY 11973, USA \\ ${ }^{7}$ Department of Physics, McGill University, Montreal, Quebec, H3A 2T8, Canada \\ ${ }^{8}$ Department of Physics and State Key Laboratory of Nuclear Physics and Technology, Peking University, Beijing 100871, China \\ ${ }^{9}$ Department of Modern Physics, University of Science and Technology of China, Hefei, Anhui 230026, China
}

\begin{abstract}
Heavy-ion collisions at very high colliding energies are expected to produce a quark-gluon plasma (QGP) at the highest temperature obtainable in a laboratory setting. Experimental studies of these reactions can provide an unprecedented range of information on properties of the QGP at high temperatures. We report theoretical investigations of the physics perspectives of heavy-ion collisions at a future high-energy collider. These include initial parton production, collective expansion of the dense medium, jet quenching, heavy-quark transport, dissociation and regeneration of quarkonia, photon and dilepton production. We illustrate the potential of future experimental studies of the initial particle production and formation of QGP at the highest temperature to provide constraints on properties of strongly interaction matter.
\end{abstract}

\section{INTRODUCTION}

The fundamental theory of strong interactions among quarks and gluons is Quantum Chromodynamics (QCD). Because of the non-Abelian nature of the strong interaction caharacterized by the $S U(3)$ gauge symmetry in QCD, quarks and gluons are confined within the realm of hadrons which are the only stable vacuum excitations. The approximate chiral symmetry among light quarks is spontaneously broken in the vacuum giving rise to non-zero quark condensates and the light pions as Goldstone bosons. The approximate conformal symmetry is also broken by quantum effects leading to a non-vanishing gluon condensate and a running strong coupling constant. Under conditions of extremely high temperature and/or density, one expects the boundary between hadrons to disappear and quark and gluon degrees of freedom are liberated to form a new state of matter called quark gluon plasma (QGP). According to lattice-discretized numerical studies of QCD (LQCD) [1, a rapid cross-over from hadronic matter to QGP occurs around a pseudo-critical temperature $T_{\mathrm{c}} \approx 155 \mathrm{MeV}$ at zero baryon chemical potential, characterized by restoration of the chiral symmetry. Below $T_{\mathrm{c}}$, quarks and gluons are confined in color-neutral hadrons in the form of a hadronic resonance matter. These hadrons melt during the deconfinement phase transition. At temperatures above $T_{\mathrm{c}}$ quarks and gluons can roam freely throughout a volume much larger than the nucleon size. The deconfinement phase transition is caused by breaking of the $Z_{3}$ center symmetry (which becomes exact in pure gauge QCD, i.e., without quark fields) at high temperature, which is characterized by a rapid change of the corresponding order parameter, the expectation value of the Polyakov loop.

Such a new state of matter of very high temperatures and densities prevailed in the early Universe as the quark epoch from $10^{-12}$ to $10^{-6}$ seconds after the Big Bang. It might still exist today in compact stellar objects such as neutron stars. In order to create this new state of matter in the laboratory, one accelerates two heavy nuclei close to the speed of light and collides them head-on. In these high-energy heavy-ion collisions, a large fraction of the colliding energy is converted into an initial matter of extremely high temperatures and densities, well beyond the phase transition region to form a QGP. Currently, two major facilities for high-energy heavy-ion collision experiments are being operated, the Relativistic Heavy-Ion Collider (RHIC) at Brookhaven National Laboratory (BNL) and the Large Hadron Collider (LHC) at the European Organization for Nuclear Research (CERN). From its start in 2000 until 2010, RHIC was the highest-energy heavy-ion collider in the world. In November 2010 the LHC took the lead as the heavy-ion collider running at the highest energy.

Remarkable discoveries have been made at RHIC since commencing its operation in 2000 [2 4], with multiple evidence pointing at the formation of a strongly-coupled QGP (sQGP) in central $\mathrm{Au}+\mathrm{Au}$ collisions at its maximum energy. One surprising discovery is that the hot and dense QCD matter created in relativistic heavy-ion collisions develops a strong collective flow characteristic of a strongly-coupled liquid, rather than of a weakly-coupled gas 
of quarks and gluons. In fact, the shear viscosity to entropy density ratio extracted from comparisons between experimental data and viscous hydrodynamic calculations is so low [5] that it has been termed "the perfect liquid". The second discovery at RHIC is the observation of substantial jet quenching [6], indicating that the matter is virtually opaque to energetic quarks and gluons. Differences in the yields and flow of baryons versus mesons indicate that hadron formation at intermediate transverse momenta proceeds via coalescence of constituent quarks, providing evidence for partonic collectivity in the observed hadron spectra [3. In fact, even heavy quarks were found to exhibit substantial collectivity and suppression indicating their approach to thermalization with a small diffusion coefficient in the strongly interacting medium [7]. The STAR experiment has also identified anti-hypertriton and anti-alpha production in $\mathrm{Au}+\mathrm{Au}$ collisions, the first ever observation of an anti-hypernucleus and anti-alpha [8].

With more than one order of magnitude higher colliding energy, many of the proposed signals for the QGP became much stronger and easier to observe at the LHC [9]. The dense matter created in heavy-ion collisions at LHC energies is much hotter and has a longer lifetime of its dynamical evolution. The QGP matter has also a smaller net baryon density as compared to that at RHIC. With increased colliding energy, the rates of hard processes are much higher than at RHIC making them much better and easily accessible probes of the QGP matter. Recent experimental data from heavy-ion collisions at LHC unambiguously confirmed all experimental evidences of the QGP as first observed at RHIC [10. The collective phenomena as manifested in anisotropic flows and a ridge structure with a large pseudorapidity gap in hadron production yields in the most central $\mathrm{Pb}+\mathrm{Pb}$ collisions point to a $\mathrm{QGP}$ at high temperatures with small specific shear viscosity. Jet quenching phenomena are clearly observed with jet energies up to hundreds of $\mathrm{GeV}$ both in the single-inclusive hadron spectra and reconstructed jets. The mass dependence of the quark energy loss is observed for the first time according to high $p_{T}$ suppression of charm mesons and non-prompt $J / \psi$ originating from bottom mesons. The centrality dependence of $J / \psi$ production clearly shows the increasing fraction of $J / \psi$ 's from recombination charm and anti-charm quarks in the QGP medium. Recent data also bear strong evidence for collectivity in high-multiplicity events of $\mathrm{p}+\mathrm{Pb}$ collisions at the LHC.

In the near future, the focus of heavy-ion collisions at RHIC and LHC will be on a quantitative characterization of the strongly coupled QGP using rare probes such as large transverse momentum jets, heavy flavor particles, real and virtual photons and quarkonia states. Studies of collective phenomena using detailed multiple particle correlations can provide precision constraints on the bulk transport coefficients of the QGP. Since existing RHIC and LHC data have already provided tantalizing hints on the weakening of the interaction strength both among bulk partons [11, 12] and between hard probes and the bulk medium [13, it will be extremely interesting to see whether such trends continue at future even higher collider energies and eventually reach the weakly interacting scenario as predicted by pQCD.

Given the state of the accelerator technology and interests in particle physics going beyond the discovery of the Higgs boson, new proposals for hadron and heavy-ion colliders at tens of $\mathrm{TeV}$ center of mass energy per nucleon pair have been envisioned [14, 15. One can address many important questions in future heavy-ion collision experiments in the energy range from tens to hundreds of TeV. These include:

(a) What is the equation of state (EoS) for the strongly interacting matter at high temperatures? Do effects of charm quarks start to become significant in the EoS?

(b) What is the thermalization mechanism, and how does the thermalization time depend on the colliding energy?

(c) What are the transport properties of strongly interacting matter at the highest temperatures probed by highenergy jets and collective phenomena? Are they approaching the weak coupling values as predicted by perturbative QCD?

(d) What is the nature of the initial state and its fluctuations in nuclear collisions?

(e) Can we find other exotic hadrons or nuclei such as light multi- $\Lambda$ hyper-nuclei, bound states of $(\Lambda \Lambda)$ or the $\mathrm{H}$ di-baryon?

(f) What are the fundamental symmetries of QCD at high temperatures? How does the restoration of the spontaneously broken chiral symmetry manifest itself in the electromagnetic radiation from the medium? Is the axial $U_{A}(1)$ symmetry effectively restored and what are the possible consequences in the hadron yields?

The answers to these important questions in strong interactions rely on both theoretical advances and experimental programs of high-energy electron-nuclei (proton) and heavy-ion collisions at future high-energy collider facilities. In this report, we will give a brief review of the physics potentials of heavy-ion collision at energy scales of tens or hundreds of $\mathrm{TeV}$. The scope of this report is limited to a few selected topics listed above. A more comprehensive report will need a much more concerted and dedicated effort.

\section{QCD AND STRONG INTERACTION MATTER}

The quantum chromodynamics (QCD), as a non-Abelian quantum gauge field theory, has been very successful in describing the strong interaction among quarks and gluons that are the fundamental constituents of visible matter in nature. The asymptotic freedom of QCD at short distances renders the possibility of calculating hard processes 
via perturbative methods. On the other hand, its non-perturbative features at long distances are only systematically computable using numerical simulations in a path-integral representation of QCD. Many of our current theoretical understanding of properties of dense matter at high temperature and baryon density are based on lattice QCD. Though experiments at RHIC and LHC have confirmed the existence of a new form of matter, strongly coupled quark-gluon plasma (sQGP), in relativistic heavy-ion collisions, its properties is not yet fully understood. This requires future efforts from both experimental and theoretical studies. In addition, lattice QCD calculations can provide crucial inputs to phenomenological studies of QGP properties.

Lattice QCD is a discretized version of QCD in the Euclidean space and time which reproduces QCD in the continuum limit when the lattice spacing goes to zero. Most lattice QCD calculations which are relevant to heavy-ion collisions have been performed using non-chiral fermions which recover the flavor or chiral symmetry of QCD only in the continuum limit, e.g. staggered and Wilson fermions. Chiral fermions are generally much more expensive to work with. However, with continued increase of the available computing power owing to Moore's law, these actions are also currently used and start to produce interesting results in QCD thermodynamics, e.g. the confirmation of the value of the crossover temperature $T_{c}$ [16] and investigations of the restoration of $U(1)_{A}$ symmetry [17-19].

\section{A. QCD transition and QCD equation of state}

The equation of state (EoS) of QCD matter contains information about the change of degrees of freedom in different regimes of temperature and baryon density. It is one of the important ingredients to model the evolution of the fireball produced in heavy-ion collisions through classical hydrodynamic equations. Computation of the QCD EoS has been one of the major goals in the field of lattice QCD since 1980 [20]. At zero baryon number density it has been shown very recently with lattice calculations for $N_{f}=2+1$ that the QCD equation of state obtained from the HotQCD and Wuppertal-Budapest collaborations by using two different discretization schemes agree very well [21, 22. Shown in Fig. 1 are energy density, entropy density and pressure as functions of temperature from the HotQCD Collaboration [21] (shaded bands). There is apparently a rapid transition from low to high temperature. It has been established from the analysis of chiral condensates that this transition in QCD with its physical mass spectrum is a rapid crossover at zero baryon density. The pseudo critical temperature of the QCD transition is confirmed to be $T_{c} \simeq 155 \mathrm{MeV}$ [16, 23, 24]. Below and around this crossover, the EoS can be described well by a hadron resonance gas model (solid lines). In the high temperature region, lattice QCD calculations of EoS and other observables, e. g. fluctuations of conserved charges can be compared to perturbative calculations [21, 25] 27, Such comparisons can provide the window of applicability for perturbation calculations and test whether the system is in the weakly coupled regime at high temperatures. In the case of $N_{f}=2+1+1 \mathrm{QCD}$, the inclusion of charm quarks may have some effects on the QCD equation of state which might be noticeable at higher temperatures reached in heavy-ion collisions at $30 \mathrm{TeV}$ scale [28, 29].

There is also some evidence that 2 or $2+1$-flavor QCD in the "chiral" limit, i.e. vanishing light quark masses with the strange quark mass being at its physical value, is second-order and belongs to the universality class of the threedimensional $O(N)$ spin models [30, 31]. If confirmed, this would be in accordance with the picture of Pisarski and Wilczek 32. However, existing studies of $O(N)$ scaling have been performed on rather coarse lattices with staggered fermion actions that are no longer state-of-the-art. They lead to large taste violations. Therefore the order of the QCD phase transition in the chiral limit is still under debate and arguments in favor of a first-order transition have been put forward 33 .

Properties of light mesons (e.g. $\rho$ ) and heavy quarkonia (e.g. $J / \psi$ and $\Upsilon$ ) as measured via the dilepton channel can serve as useful probes for the chiral symmetry restoration and deconfinement transition in the QCD medium, respectively. Theoretical study of these hadron properties at finite temperature requires the computation of two-point correlation functions on the lattice and extraction of hadron spectral functions. These hadron spectral functions are directly related to thermal dilepton rates, the dissociation of quarkonia states as well as transport properties of the medium, e.g. electrical conductivity and heavy quark diffusion coefficients.

The most current lattice QCD study of hadron spectral functions suggests that all charmonia dissociate at $T \gtrsim 1.5 T_{c}$ in gluonic plasma [34]. Very recent lattice QCD studies including dynamic quarks on screening mass and spectral functions suggest the same picture [35, 36. Due to the large value of heavy bottom quark mass, a direct study of bottomonia is very hard on the lattice since the lattice spacing $a$ has to be much smaller than the inverse of the heavy quark mass. Effective theories, e.g. non-relativistic QCD (NRQCD), have been put on the lattice to study properties of bottomonia. It has been found that all $\mathrm{S}$ wave states exist at temperatures up to at least $2 T_{c}$ and $\mathrm{P}$ wave states melt just above $T_{c}$ [37 41]. However, a different observation is found in Ref. [42] that $\mathrm{P}$ waves states might stay bounded at higher temperatures above $T_{c}$ by using a new inversion method. It has also been realized that the potential of static quarks in the medium is complex [43 45] whose computation on the lattice has been carried out [46-49]. 


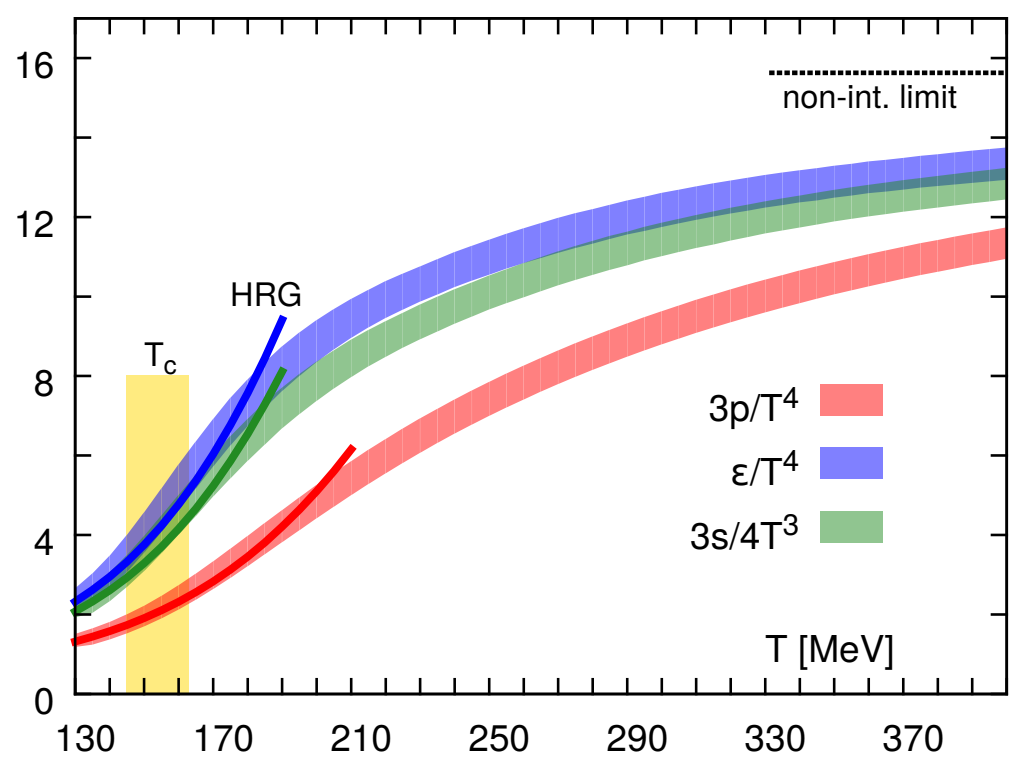

Figure 1: The pressure, energy and entropy density (scaled by $T^{4}$ ) as functions of the temperature from lattice QCD calculation by the HotQCD Collaboration [21] (shaded bands) as compared to hadron resonance gas (HRG) model results (solid lines).

The fate of heavy-light mesons or baryons also reflects the change of relevant degrees of freedom in the strong interaction matter. For instance, the abundance of strange hadrons is considered as one of the signals for the formation of QGP. Investigations of fluctuations and correlations of electrical charge and baryon number with strangeness and charm found that both open strange and open charm hadrons start to dissociate in the temperature region of the chiral crossover 50,52 .

As proposed recently in Ref. [53], hadron chemical freeze-out temperatures and baryon chemical potentials can be determined by matching lattice QCD computations with those measured in heavy-ion collisions. An upper band of freeze-out temperature is found to be $148 \pm 4 \mathrm{MeV}$ [54]. An indirect evidence of experimentally yet unobserved open strange and open charm hadrons has been found [51, 55]. These unobserved hadrons bring down the freeze-out temperature in the strange hadron sector by $\sim 5-8 \mathrm{MeV}[55]$.

\section{B. Transport coefficients}

Transport properties of the hot QCD medium are also the focus of future experimental studies through collective phenomena of both light and heavy flavor hadrons and electromagnetic emissions. Currently there are only a limited number of results on transport coefficients from lattice-QCD calculations with dynamical quarks. Most calculations have been performed in the quenched limit at vanishing net-baryon number density [56 [58]. It proves difficult to extract transport coefficients directly from imaginary-time two-point correlation functions. Currently, the maximum entropy method (MEM) is a commonly used technique to achieve this goal [59]. The determination of the electrical conductivity and the heavy-quark diffusion coefficient in full QCD is rather straightforward and is mainly limited by computational resources. However, the determination of fluid-dynamical transport coefficients, e.g. viscosities, is hampered by large noise-to-signal ratios. For QCD in the quenched approximation, noise reduction techniques are known and are applied while for full QCD computations such algorithms still need to be developed.

Electrical conductivity has been computed in the continuum limit in quenched QCD at three temperatures above $T_{c}$ 60, 61]. Recently computation has also been performed on the lattice with dynamic quarks 62 64. The charmquark diffusion coefficient has been obtained at one value of the lattice cutoff and three temperatures in the deconfined phase [56]. Currently, there are no lattice results on bottom-quark diffusion coefficients which are very important in heavy-quark physics at LHC energies and beyond. The heavy-quark diffusion coefficients have also been studied on the lattice by measuring proposed observables in heavy-quark effective theory [65]. Results on heavy-quark diffusion coefficients obtained in this approach are close to the charm-quark diffusion coefficients 66 68. However, most of these results are also obtained at a finite lattice cutoff, so a reliable extraction of diffusion coefficients needs to be performed.

Shear and bulk viscosities have been calculated a few years ago on rather coarse and small lattices, without a 
continuum extrapolation 69,70 . In order to obtain better results, the number of gauge field configurations needs to be increased by an order of magnitude. However, algorithms like multi-level updates to improve the signal-to-noise ratio [71] of two-point correlators of the energy-momentum tensor currently used in the quenched approximation are not applicable in full QCD. Recently, there have been efforts [2] 74 to determine some of the 2nd-order transport coefficients from a first-principles calculation on lattice.

\section{BULK PROPERTIES OF MATTER IN HEAVY-ION COLLISIONS}

In the study of QGP properties in high-energy heavy-ion collisions, the space-time evolution of the bulk matter underpins all experimental and phenomenological studies since it will affect all the expected final observables from which one extracts medium properties of the QGP. Whether it is an effective theory such as relativistic hydrodynamics or a Monte Carlo model for parton and hadron transport, one always needs the basic information of initial parton production. The initial parton production determines the initial energy density or temperature at the thermalization time and its fluctuation in both transverse area and longitudinal direction. Given these initial conditions, one can then use the hydrodynamical model or parton-hadron transport model for the space-time evolution of the bulk medium. Through comparisons between hydrodynamic or transport results and experimental data on the final hadron spectra and their azimuthal anisotropy or multiple hadron correlations, one can extract values of the bulk transport coefficients such as shear and bulk viscosity. For the study of other hard and electromagnetic signals, one also has to reply on the space-time evolution of the bulk medium to understand the experimental measurements and extract medium properties such as initial temperature, flow velocity and jet transport coefficients.

\section{A. Multiplicity}

The mechanism of initial parton production has been one of the fundamental problems in heavy-ion collisions and strong interaction in general. It is determined by the properties of strong interaction at high energy where non-linear aspects of QCD are at play and it is also the focus of research at the future electron-ion colliders (EIC). Shown in Fig. 2 is the charged hadron multiplicity in $p+p(\bar{p})$ collisions as a function of the colliding energy as extrapolated from experimental data at Fermilab Tevatron [75, BNL RHIC 76] and CERN LHC 77 to very high energies. This extrapolation is also consistent with HIJING calculations 78 in which the rise of the multiplicity in the central rapidity region at high colliding energy is mainly caused by the increase of gluonic mini-jet production with the large initial gluon distribution inside the beam proton at small momentum fraction.

There are currently two types of pQCD based models for the description of initial parton production in heavy-ion collisions. HIJING Monte Carlo model [78, 80, employs the Glauber model for multiple interaction in high-energy nucleon-nucleus and nucleus-nucleus collisions. It includes both the incoherent hard and semi-hard parton scattering that are described by pQCD and the coherent soft interaction via excitation of remanent strings between valence quarks and diquarks. Initial parton production from incoherent hard or semi-hard parton scatterings is proportional to the number of binary nucleon-nucleon collisions $N_{\text {coll }}$ while the soft parton production from string excitation is proportional to the number of participant nucleons $N_{\text {part }}$ in a given centrality. One should also take into account the impact-parameter-dependent nuclear modification of parton distributions in the semi-hard parton interaction. This will introduce additional impact-parameter dependence of the parton production in the hard or semi-hard parton scattering. The final centrality dependence of the initial parton multiplicity from both soft and semi-hard processes will therefore be a linear combination of $N_{\text {part }}$ and $N_{\text {coll }}$.

The average number of participant nucleons or wounded nucleons $N_{\text {part }}$ in heavy-ion collisions as a function of the impact-parameter can be calculated within the Glauber model in terms the overlapping functions of two nuclei [81. It can reach the limit of the total number of nucleons within the overlap region of two colliding nuclei. It therefore has a very weak energy dependence in very high energies. The number of binary collisions depends almost linearly on the total inelastic cross section and therefore has a strong energy dependence. Correspondingly, the final hadron multiplicity per participant pair should increase faster as a function of energy as compared to $p+p$ collisions. Similarly, the final hadron multiplicity in the central rapidity region per participant pair at fixed colliding energy should increase with $N_{\text {part }}$ towards more central collisions as shown by the HIJING simulations in Fig. 3 . The exact behavior of the final hadron multiplicity per participant pair as a function of the centrality or $N_{\text {part }}$ is controlled mainly by the impact-parameter dependence of the parton shadowing in heavy nuclei which can also be addressed by experiments at future high energy electron-ion colliders.

The second type of models for initial particle production is based on the approach of interacting semi-classical gluonic fields or the Color Glass Condensate model [82. There are many variants of the model including KLN [83 85], rcBK [86, 89] and IP-Glasma [90, 92]. One can calculate initial gluon multiplicity in heavy-ion collisions and assume 


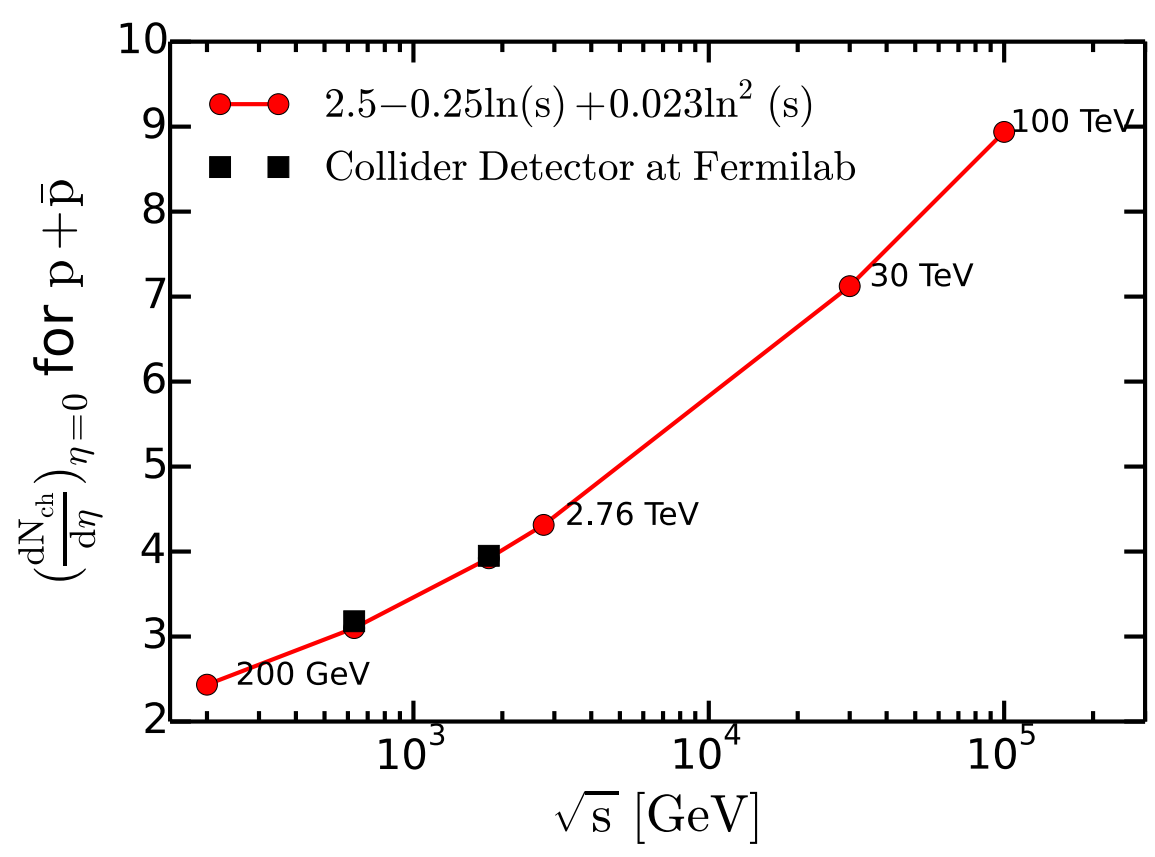

Figure 2: The charged multiplicity for $p+p(\bar{p})$ collisions as a function of the colliding energy, as extrapolated from experimental data at Fermilab Tevatron, BN RHIC and CERN LHC.

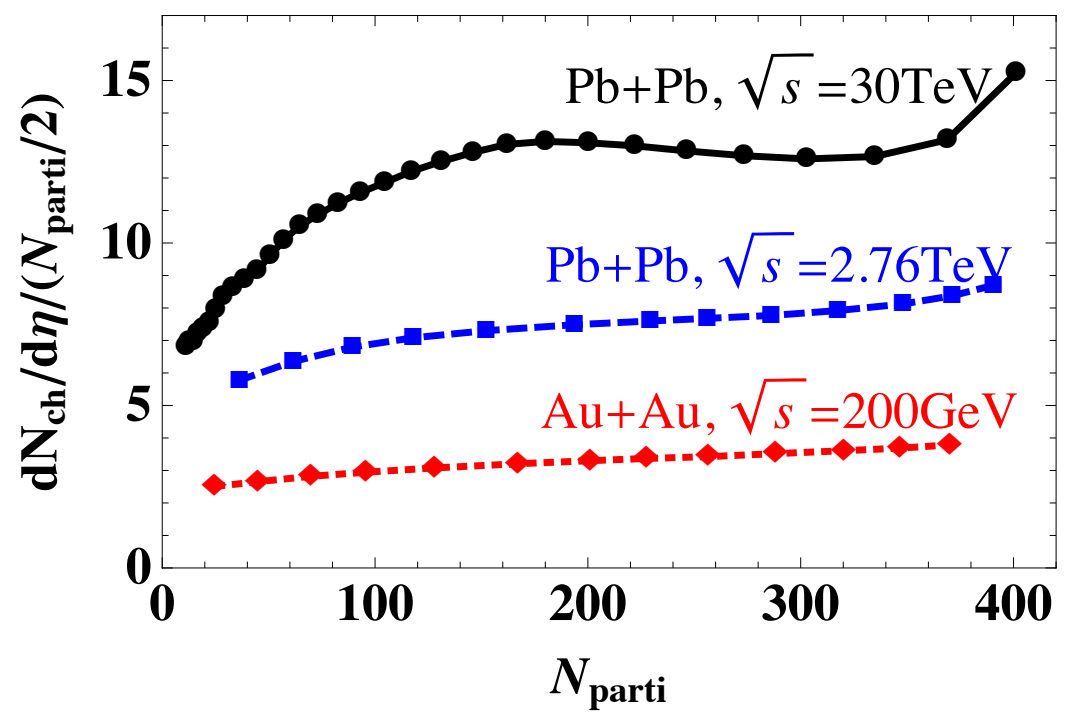

Figure 3: Rapidity density of charged hadrons in the central rapidity per participant pair as functions of the number of participant nucleons in $\mathrm{Au}+\mathrm{Au}$ collisions at $\mathrm{RHIC}, \mathrm{Pb}+\mathrm{Pb}$ collisions at LHC and at $\sqrt{s}=30$ TeV, from HIJING Monte Carlo simulations.

parton-hadron duality to obtain the final hadron multiplicity. The IP-Glasma model combines the impact parameterdependent saturation model for high-energy nucleon and nuclear wave function with classical Yang-Mills dynamics of Glasma fields in heavy ion collisions. It can be used to estimate the initial energy density event by event. In the rcBK model, the $k_{T}$-factorization is assumed which involves an integral over unintegrated gluon distributions whose evolution can be obtained by solving the nonlinear Balitisky-Kovchegov (BK) equation with the running coupling kernel (rcBK) [86 89].

Shown in Fig. 4 is the centrality dependence of charged particle multiplicity at three collision energies using the rcBK model (open symbols) which can reproduce experimental results at RHIC and LHC energies (solid symbols). 


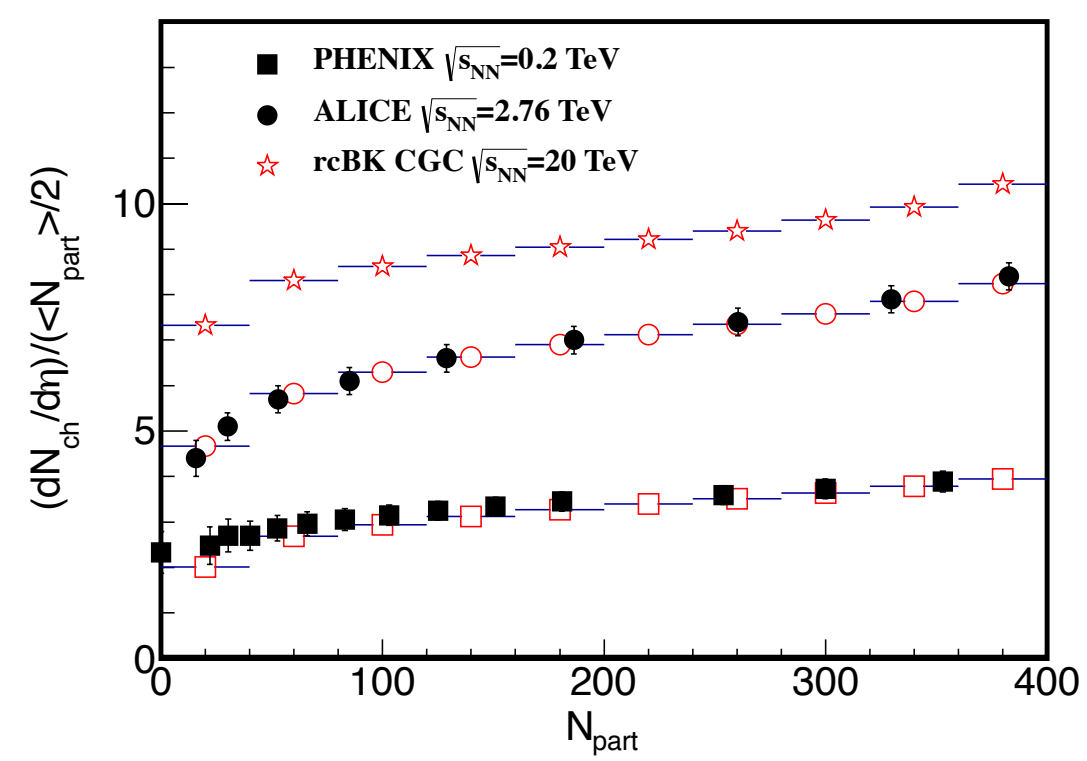

Figure 4: Centrality dependences of charged particle multiplicity at $\sqrt{s_{N N}}=0.2,2.76$ and 20 TeV, respectively from the rcBK model [86] 89].

\begin{tabular}{r|l|l|l|l}
$\sqrt{s_{N N}}[\mathrm{TeV}]$ & $\varepsilon_{0}\left[\mathrm{GeV} / \mathrm{fm}^{3}\right]$ & $T_{0}[\mathrm{MeV}]$ & $\tau_{0}[\mathrm{fm}]$ & $d N_{\mathrm{ch}} / d \eta$ \\
\hline $0.20(\mathrm{Au}+\mathrm{Au})$ & 30 & 357 & 0.6 & 745 \\
$2.76(\mathrm{~Pb}+\mathrm{Pb})$ & 77 & 449 & 0.6 & 1700 \\
$30(\mathrm{~Pb}+\mathrm{Pb})$ & 136 & 517 & 0.6 & 2700
\end{tabular}

Table I: The initial energy density $\varepsilon_{0}$, temperature $T_{0}$ at the center of heavy-ion collisions, thermalization time $\tau_{0}$ and the final charged hadron rapidity density at different colliding energies.

The hadron multiplicity in the most central $\mathrm{Pb}+\mathrm{Pb}$ collisions at $\sqrt{s}=20 \mathrm{TeV}$ from the rcBK model estimate is comparable to the HIJING estimate (shown in Fig. 3 is for $\sqrt{s}=30 \mathrm{TeV}$ ). Notice that a cross section parameter of hard valence charges is assumed as energy-independent $\left(\sigma_{0}=4.2 \mathrm{fm}^{2}\right)$. If an energy-dependent cross section parameter is used, one will get a flatter curve for the centrality dependence. The mechanism and consequences of gluon saturation is also one of the main topics at future high-energy electron-ion colliders.

Together with the transverse distribution of participant nucleons and binary collisions, the above models for initial particle production can provide the initial energy density distributions which can fluctuate from event to event. These fluctuating initial energy density distributions in turn will provide the initial conditions for hydrodynamic or transport evolution of the bulk matter in heavy-ion collisions. The initial energy density and temperature at the center of heavy-ion collisions at an initial thermalization time $\tau_{0}=0.6 \mathrm{fm} / c$ are listed in Table I

\section{B. Collective expansion and anisotropic flow}

One of the evidences for the formation of sQGP in heavy-ion collisions at RHIC and LHC is the observation of strong anisotropic flow due to collective expansion driven by the initial high energy density and pressure in the overlapping region of the collisions [2, 3]. During the last decade of both experimental and theoretical exploration of this phenomenon, a rather detailed picture of the collective expansion of the anisotropic fireball in heavy-ion collisions emerges. During the early stage of high-energy heavy-ion collisions, the local transverse energy density is governed by the initial wave functions of the colliding nuclei, the interaction strength of beam partons and the quantum process of parton production. These different aspects of initial parton production determine the event-by-event transverse as well as longitudinal energy density distributions during the early stage of the heavy-ion collisions. Due to the thermalization processes whose mechanism is still under intense theoretical investigation [93, these initial states of fluctuating energy density distributions achieve local equilibrium and the subsequent collective expansion can be 

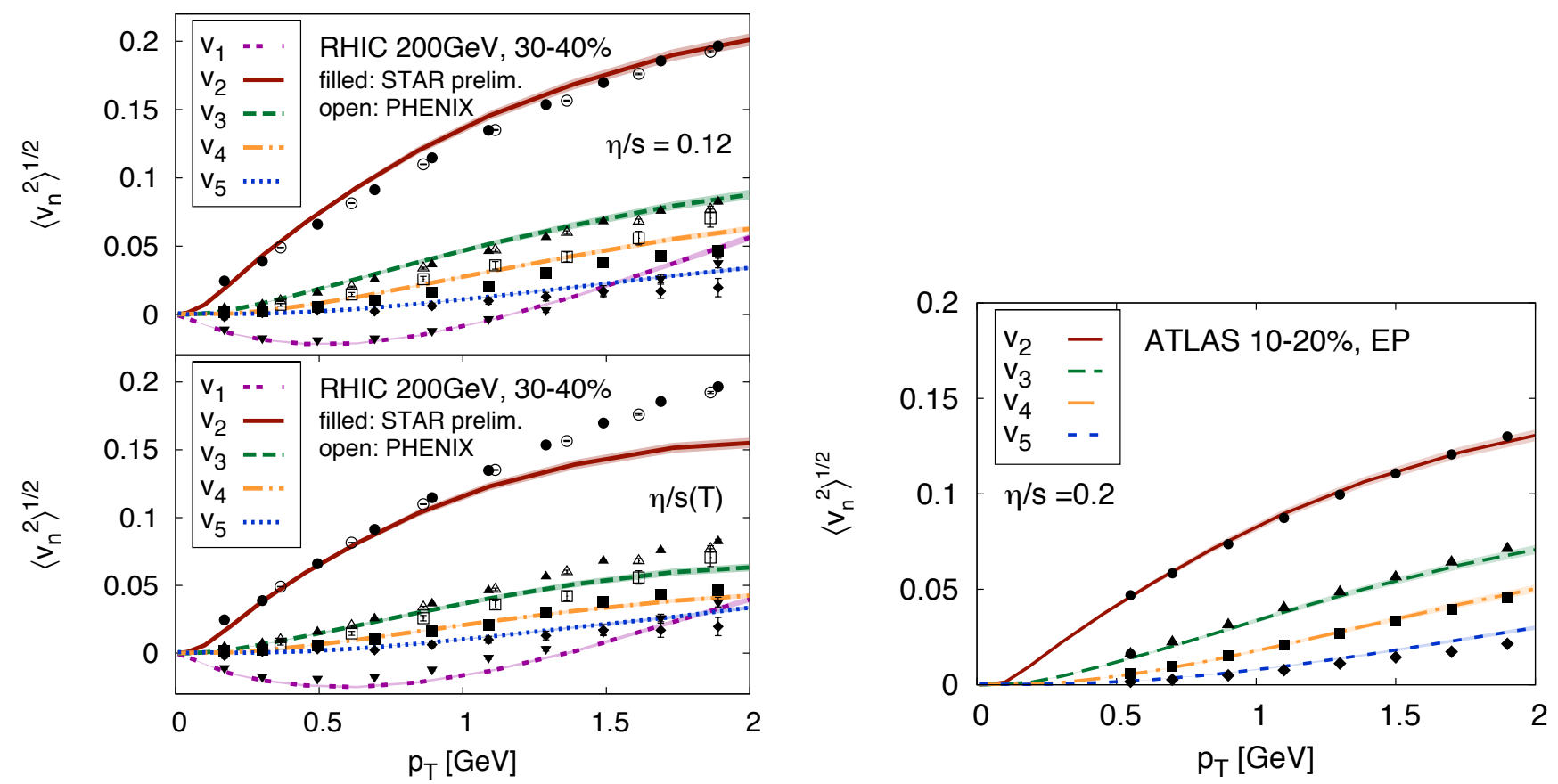

Figure 5: Anisotropic flows as functions of $p_{T}$ from viscous hydrodynamic model simulations with event-by-event fluctuating initial condition from the IP-Glasma model [12] as compared to experimental data from PHENIX [99] and STAR [100] at RHIC and ATLAS [101] at LHC.

approximately described by relativistic viscous hydrodynamic equations with an effective EoS as obtained from lattice QCD results [94. After hydrodynamic expansion over a finite period of time, the spatial anisotropies of the initial energy density distributions are converted into anisotropies of the final hadron spectra in momentum space [95]. One can characterize the momentum anisotropies in terms of the Fourier coefficients of the final hadron azimuthal distribution or two-particle azimuthal correlation in each event. One normally refers to these Fourier coefficients as anisotropic flows $v_{n}$ with the corresponding order $n$ of the Fourier expansion. Comparisons of the experimental measurements of the anisotropic flows at RHIC and results from viscous hydrodynamic model simulations point to rather small values of the shear viscosity to entropy density ratio $\eta / s$ [96, 97, that is very close to the quantum mechanics bound [98].

Shown in Fig. 5 are the calculated anisotropic flows from the state of art $2+1 \mathrm{D}$ viscous hydrodynamic simulations [12] that employs the IP-Glasma model for initial gluon production with both event-by-event geometric fluctuations in nucleon positions and the sub-nucleon color-charge fluctuations. Hydrodynamic results describe extremely well the experimental data on the anisotropic flows up to the fifth order in heavy-ion collisions at both RHIC and LHC. There is also an indication that the shear viscosity to entropy ratio decreases slightly from RHIC to LHC. This points to the direction of theoretical estimate that the QGP at higher temperatures might transit from a strongly coupled to weakly coupled one as described by pQCD calculations. Heavy-ion collisions at the very high energy region can reach even higher initial temperatures (see Table I) and therefore approach closer to such a weak coupling limit.

Assuming the same values of shear viscosity to entropy density ratio as in heavy-ion collisions at the LHC, the differential anisotropic flows at $\sqrt{s}=20-30 \mathrm{TeV}$ should remain roughly the same. However, due to increased radial flow and the flattening of the transverse momentum spectra, the integrated anisotropic flows should continue to increase with the colliding energy. Shown in Fig. 6 are differential anisotropic flows calculated from $3+1 \mathrm{D}$ ideal hydrodynamic simulations [102] with fluctuating initial conditions from HIJING [78 80] and AMPT [103] models for $20-30 \%$ central $\mathrm{Pb}+\mathrm{Pb}$ collisions at $30 \mathrm{TeV}$ (solid lines) as compared to those at $2.76 \mathrm{TeV}$ (dashed lines). For over all normalization of the final hadron multiplicity we have rescaled the initial energy density from the AMPT model by a factor so that the multiplicity in $20-30 \%$ central $\mathrm{Pb}+\mathrm{Pb}$ collisions at $30 \mathrm{TeV}$ is close to most central $\mathrm{Pb}+\mathrm{Pb}$ collisions at $\sqrt{s_{N N}}=2.76 \mathrm{TeV}$. In these initial conditions fluctuations in the longitudinal direction are also considered that should affect the final state anisotropic flow in the central rapidity region [102]. Harmonic flow coefficients $v_{n}$ at both energies show a normal ordering that decreases with the order of harmonics at the same $p_{T}$. The second harmonic caused mainly by the initial geometry for non-central collisions at $30 \mathrm{TeV}$ is slightly higher than at $2.76 \mathrm{TeV}$ because 


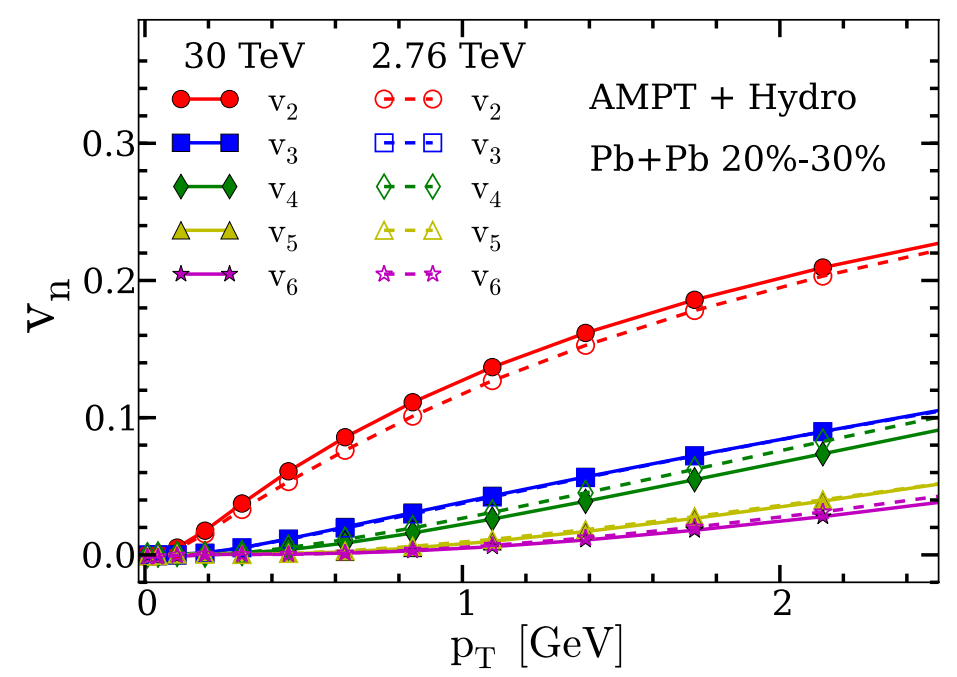

Figure 6: Anisotropic flows $v_{n}$ for $20-30 \%$ central $\mathrm{Pb}+\mathrm{Pb}$ collisions at $\sqrt{s_{N N}}=30 \mathrm{TeV}$ from $3+1 \mathrm{D}$ ideal hydrodynamic simulations with full fluctuating initial conditions from HIJING and AMPT model.

of increased initial energy density and longer duration of expansion. The higher harmonics which are caused by initial fluctuations remains almost the same at $30 \mathrm{TeV}$ as at $2.76 \mathrm{TeV}$. This indicates that the relative transverse fluctuations do not change at higher colliding energies.

AMPT +hydro model introduces longitudinal fluctuations which will result in decorrelation of event planes for particles with large pseudo rapidity gaps. Recent studies [104] show that the decorrelation of anisotropic flows along longitudinal direction is stronger for lower energy collisions due to bigger fluctuations.

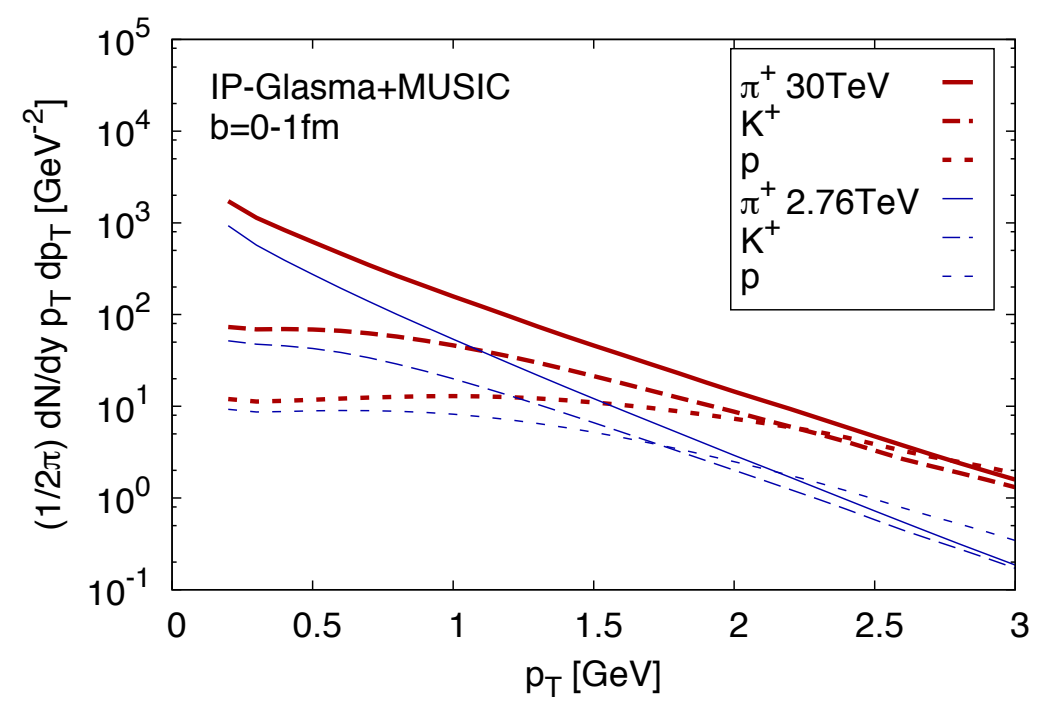

Figure 7: Transverse momentum spectra of positive pions, kaons, and protons for impact parameter $b=0-1 \mathrm{fm}$ in $2.76 \mathrm{TeV}$ (thin lines) and $30 \mathrm{TeV}$ (thick lines) $\mathrm{Pb}+\mathrm{Pb}$ collisions computed using IP-Glasma + Music with $\eta / s=0.2$.

To study the effect of shear viscosity in heavy-ion collisions at very high colliding energies, we show predictions for transverse momentum spectra and anisotropic flows $v_{n}\left(p_{T}\right)$ of pions, kaons, and protons and charged hadrons in central $(b=0-1 \mathrm{fm}) \mathrm{Pb}+\mathrm{Pb}$ collisions at $30 \mathrm{TeV}$ compared to those at $2.76 \mathrm{TeV}$, from IP-Glasma 90, 91, MUsiC simulations [12, 105]. The IP-Glasma model relates the nuclear dipole cross-sections constrained by the deeply inelastic scattering (DIS) data to the initial classical dynamics of highly occupied gluon fields produced in a nuclear collision. 


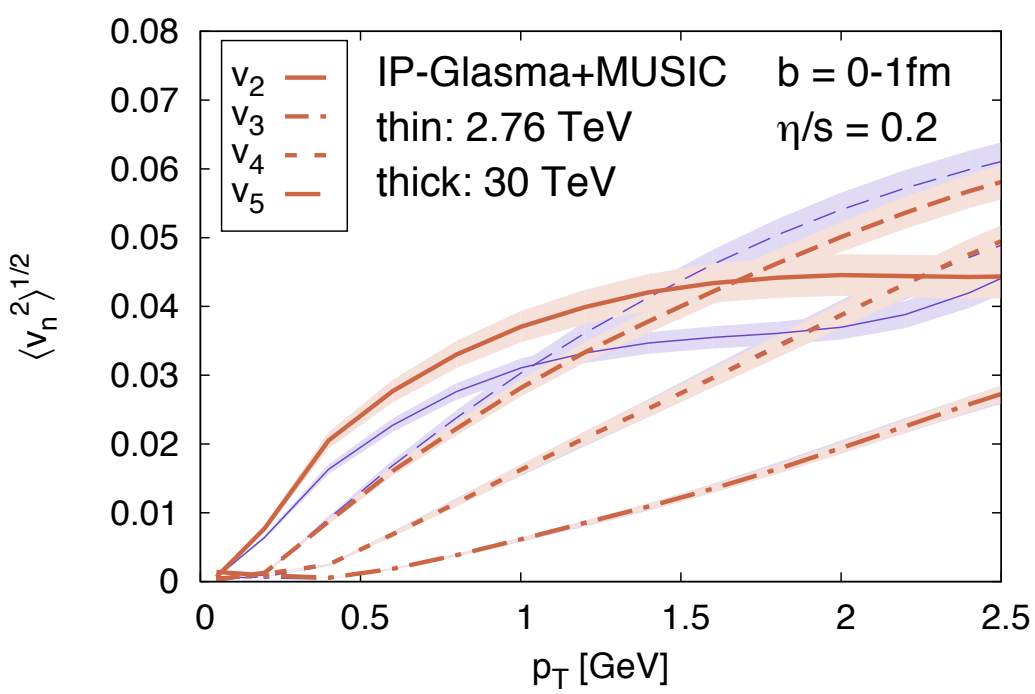

Figure 8: Root-mean-square harmonic flow coefficients $v_{n}\left(p_{T}\right)$ of charged hadrons for impact parameter $b=0-1 \mathrm{fm}$ in $2.76 \mathrm{TeV}$ (thin lines) and $30 \mathrm{TeV}$ (thick lines) $\mathrm{Pb}+\mathrm{Pb}$ collisions computed using IP-Glasma + Music with $\eta / s=0.2$.

Given an initial distribution of color charges in the high-energy nuclear wave-functions, the strong multiple scatterings of gluon fields are computed by event-by-event solutions of Yang-Mills equations. This includes both fluctuations of nucleon positions and subnucleonic color charge distributions. The scale of the resulting fluctuating structure of the gluon fields is given on the average by the nuclear saturation scale $Q_{s}$. Typically, this length scale $1 / Q_{s}$ is smaller than the nucleon size [106. A detailed description of the IP-Glasma model can be found in Refs. 90 92.

The IP-Glasma model provides the initial conditions for fluid dynamic calculations at a given time $\tau_{0}$. The initial energy density $\varepsilon$ and flow velocities $u^{\mu}$ are extracted from the gluon fields' energy-momentum tensor $T^{\mu \nu}$ at every transverse position via the relation $u_{\mu} T^{\mu \nu}=\varepsilon u^{\nu}$. The viscous part of the energy-momentum tensor is set to zero at the initial time of the fluid dynamic simulation $\tau=0.2 \mathrm{fm}$.

The fluid dynamic simulation used is the $3+1$ dimensional viscous relativistic simulation MusIC [107-109] employing a lattice equation of state with partial chemical equilibrium as described in Ref. [12] and a shear viscosity to entropy density ratio $\eta / s=0.2$, which led to a good description of the experimentally measured flow harmonics in $\mathrm{Pb}+\mathrm{Pb}$ collisions at $2.76 \mathrm{TeV}[12$. When employing IP-Glasma initial conditions the spatial dimensions are reduced to 2, assuming boost-invariance of the initial condition. The calculation of particle spectra and the analysis of the azimuthal anisotropy follows the same steps as discussed above for the ideal hydrodynamic simulations.

As shown in Fig. 7) we find that the transverse momentum spectra at $30 \mathrm{TeV}$ are significantly harder than at $2.76 \mathrm{TeV}$ and that the $p_{T}$-integrated multiplicity at mid-rapidity after viscous fluid dynamic evolution is approximately a factor of 2.8 greater. This increase of multiplicity from 2.76 to $30 \mathrm{TeV}$ is somewhat higher than other model predictions as shown in Sec. IIIA. The initial gluon multiplicity obtained in Coulomb gauge only increases by approximately a factor of 2.3. This change depends on the energy dependence of $Q_{s}$ in the IP-Saturation model as well as the implementation of the running coupling. The multiplicity scales with $\alpha_{s}^{-1}$ and we have chosen the scale of the running coupling to be the average minimum $Q_{s}$ value. The possibility to choose another scale introduces a logarithmic uncertainty on the overall multiplicity. The additional relative increase in multiplicity from $\mathrm{Pb}+\mathrm{Pb}$ collisions at $2.76 \mathrm{TeV}$ to $30 \mathrm{TeV}$ can be attributed to increased entropy production due to larger gradients and the approximately $4 \mathrm{fm} / c$ longer evolution time.

As in the ideal hydrodynamic calculations, $v_{n}$ coefficients in $\mathrm{Pb}+\mathrm{Pb}$ collisions at $\sqrt{s}=30 \mathrm{TeV}$ are not changed significantly from those at $2.76 \mathrm{TeV}$ as a function of $p_{T}$ when using a fixed shear viscosity to entropy density ratio as shown in Fig. 8. The second harmonics $v_{2}$ is slighty higher in the higher energy collisions, while all higher harmonics agree within the statistical errors shown. It would thus be a clear indication of a change of transport coefficients with collision energy, should significantly different values of the flow harmonics be measured in the $30 \mathrm{TeV}$ collisions. Precision measurements of these anisotropic flows at future very high energy heavy-ion collisions can therefore shed light on the temperature dependence of the shear viscosity to entropy ratio and whether one is approaching a weakly coupling limit as given by $\mathrm{pQCD}$ at higher colliding energies. 


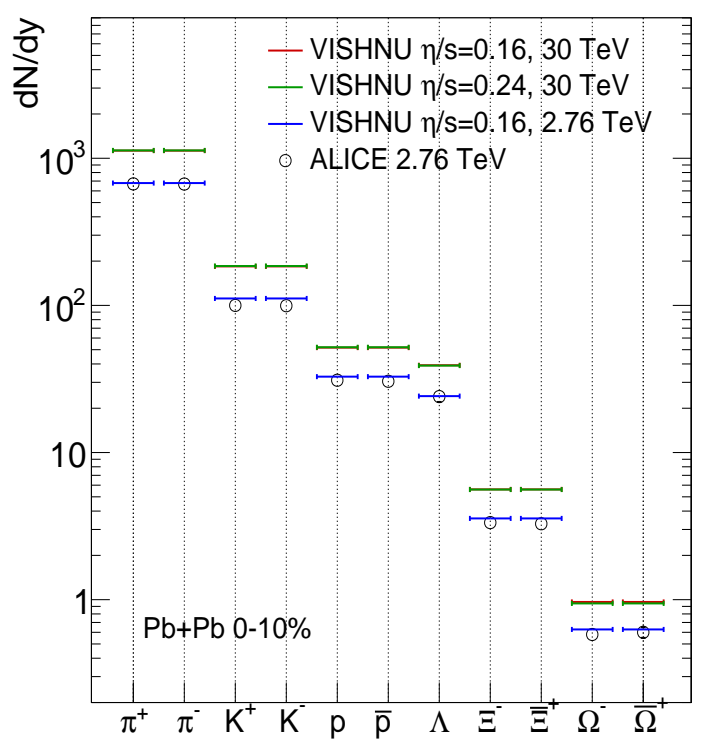

Figure 9: (Color online) Multtiplicity density, $d N_{\mathrm{ch}} / d y$, for various hadron species in the most central $\mathrm{Pb}+\mathrm{Pb}$ collisions at $\sqrt{s_{\mathrm{NN}}}=2.76 \mathrm{TeV}$ and at $\sqrt{s_{\mathrm{NN}}}=30 \mathrm{TeV}$. The VISHNU results and the ALICE measuremnts at the LHC are respectively taken from [110, and $111+113$.

\section{Flavor dependence of hadron spectra and elliptic flow}

After the QCD phase transition, the succeeding hadronic evolution involves frequent elastic, semi-elastic, inelastic collisions and resonance decays. When most of the inelastic collisions and resonance decays cease, yields of various hadrons no longer change. The system is considered to reach chemical freeze-out. Therefore, yields of soft identified hadrons can also provide information on the chemical freeze-out of the evolving dense matter. In the statistical model [114 116], the chemical freeze-out temperature $T_{\mathrm{ch}} \sim 165 \mathrm{MeV}$ and chemical potential $\mu_{b} \sim 24 \mathrm{MeV}$ are extracted from particle yields in $\mathrm{Au}+\mathrm{Au}$ collisions at the top RHIC energy [114. With $T_{\mathrm{ch}} \sim 165 \mathrm{MeV}$ and $\mu_{b} \sim 0$, the statistical model can also describe yields of many identified hadrons in $\mathrm{Pb}+\mathrm{Pb}$ collisions at $\sqrt{s_{\mathrm{NN}}}=2.76 \mathrm{TeV}$, but over-predicts the protons/antiprotons data. Recent hybrid model simulations indicated that the chemical freeze-out temperature might depend on hadron species [110, 117]. With baryon-antibaryon $(B-\bar{B})$ annihilations that delay the chemical freeze-out of baryon and antibaryons, VISHNU [97, 118] largely improves the description of protons/antiprotons data, which also fits particle yields of other identified hadron species well in $\mathrm{Pb}+\mathrm{Pb}$ collisions at the $\mathrm{LHC}[110]$.

At much higher collision energies, the created QGP fireball could reach even higher temperatures, leading to more frequent $B-\bar{B}$ annihilations in the succeeding hadronic evolution. Therefore, future measurements of soft hadron yields in $\mathrm{Pb}+\mathrm{Pb}$ collisions at $\sqrt{s_{\mathrm{NN}}}=30 \mathrm{TeV}$ will provide more information for the chemical freeze-out process of the hot QCD system. Meanwhile, measurements of spectra and elliptic flow of identified soft hadrons will help us to understand the interplay of radial and elliptic flow at much higher collision energies and provide additional constraints for the extracted QGP viscosity at higher temperatures.

In this subsection, we predict multiplicity, spectra and elliptic flow of identified hadrons in $\mathrm{Pb}+\mathrm{Pb}$ collisions at $\sqrt{s_{\mathrm{NN}}}=30 \mathrm{TeV}$, using the VISHNU hybrid model. VISHNU [97, 118, combines $(2+1)$-D relativistic viscous hydrodynamics (VISH2+1) [119, 120] for the QGP fluid expansion with a microscopic hadronic transport model (UrQMD) [121, 122] for the hadron resonance gas evolution. The transition from hydrodynamics to hadron cascade occurs on a switching hyper-surface with a constant temperature. Generally, the switching temperature $T_{\mathrm{sw}}$ is set to $165 \mathrm{MeV}$, which is close to the QCD phase transition temperature [23, 24, 123, 125]. For hydrodynamic evolution above $T_{\text {sw }}$, we use an equation of state (EoS) constructed from recent lattice QCD data [94, 126]. Hydrodynamic simulations start at $\tau_{0}=0.9 \mathrm{fm} / c$ with the MC-KLN initial conditions. For computational efficiency, we implement singleshot simulations [110, 117] with smooth initial entropy density profiles generated from the MC-KLN model through averaging over a large number of events within specific centrality bins. Considering the approximate liner relationship between initial entropy and final multiplicity of all charged hadrons, we cut centrality bins through the distribution of initial entropies obtained from MC-KLN. In these calculations, the QGP specific shear viscosity $(\eta / s)_{Q G P}$ is set to 0.16 and 0.24 . The normalization factor of the initial entropy density is tuned to fit the estimated multiplicity 


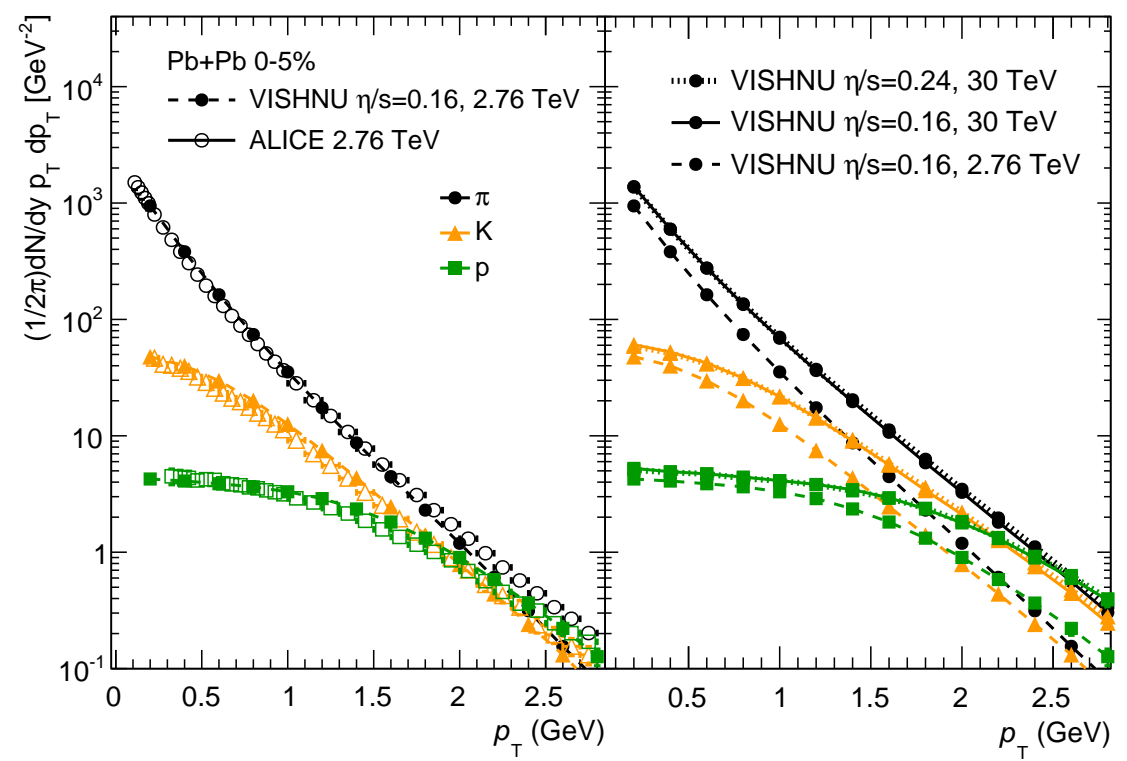

Figure 10: (Color online) Transverse momentum spectra of $\pi, K$, and $p$ in the most central $\mathrm{Pb}+\mathrm{Pb}$ collisions at $\sqrt{s_{\mathrm{NN}}}=2.76$ $\mathrm{TeV}$ and at $\sqrt{s_{\mathrm{NN}}}=30 \mathrm{TeV}$. The VISHNU results and the ALICE measurements at the LHC are respectively taken from [117. and 111.

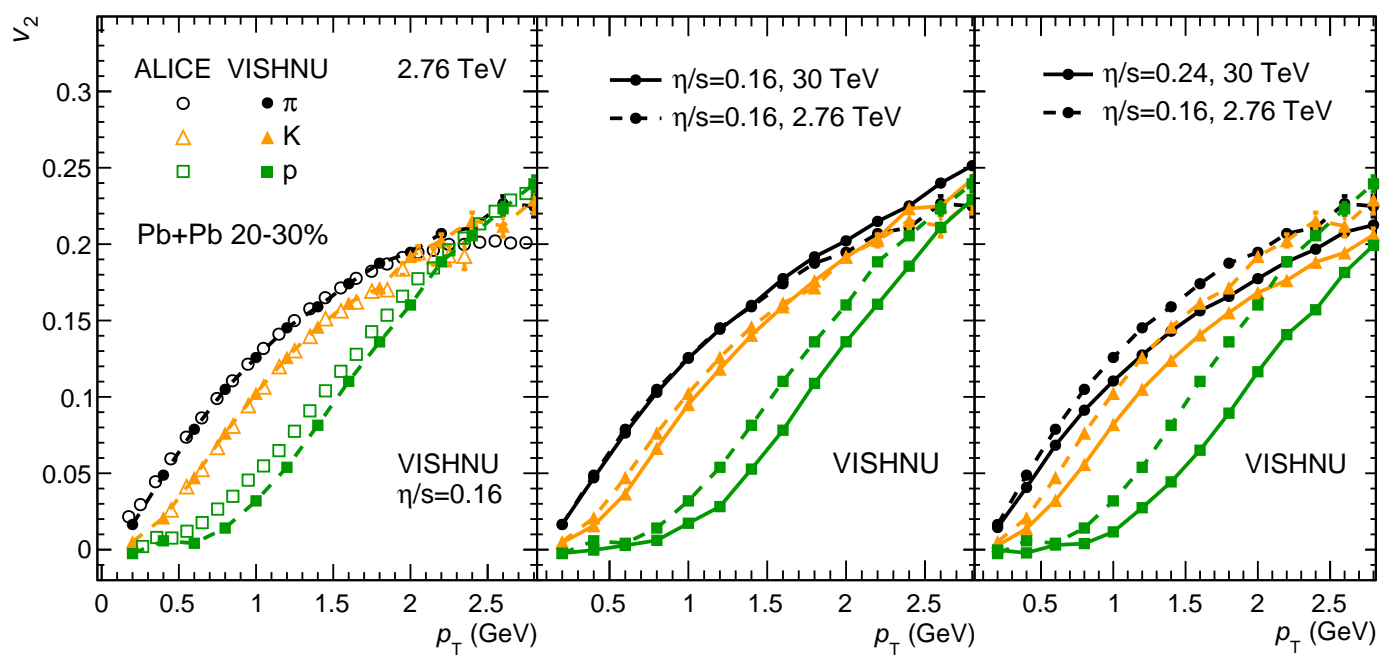

Figure 11: (Color online) Differential elliptic flow of $\pi, K$, and $p$ in semi-central $\mathrm{Pb}+\mathrm{Pb}$ collisions at $\sqrt{s_{\mathrm{NN}}}=2.76$ TeV and at $\sqrt{s_{\mathrm{NN}}}=30 \mathrm{TeV}$. The VISHNU results and the ALICE measurements at the LHC are respectively taken from [117] and [127.

density of all charged hadrons in the most central collisions $(\sim 2700)$. To simplify the theoretical calculations, we set the bulk viscosity to zero and neglect net baryon density and the heat conductivity.

Fig. 9 shows the VISHNU prediction for the multiplicity densities $d N / d y$ of $\pi, K, p, \Lambda, \Xi$, and $\Omega$ in the most central $\mathrm{Pb}+\mathrm{Pb}$ collisions at $\sqrt{s_{\mathrm{NN}}}=30 \mathrm{TeV}$. Compared with results at the LHC [110] energy, particle yields of various hadron species increase significantly in heavy-ion collisions at the future colliding energy. These results also indicate that the $B-\bar{B}$ annihilations during the hadronic evolution are significantly enhanced at the high collision energy of $30 \mathrm{TeV}$.

Fig. 10 presents VISHNU calculations for the transverse momentum spectra of $\pi, K$, and $p$ in the most central $\mathrm{Pb}+\mathrm{Pb}$ collisions at $\sqrt{s_{\mathrm{NN}}}=2.76 \mathrm{TeV}$ and at $\sqrt{s_{\mathrm{NN}}}=30 \mathrm{TeV}$. Compared with results at the LHC energy [117, the predicted spectra of $\pi, K$, and $p$ at $\sqrt{s}=30 \mathrm{TeV}$ are flatter with higher integrated yields. This result indicates that the amount of radial flow generated from $30 \mathrm{~A} \mathrm{TeV}$ collisions is larger than the one generated from collisions at 2.76 TeV.

Besides the multiplicity and spectra, we also predict the differential elliptic flow of pions, kaons and protons in semi-central $\mathrm{Pb}-\mathrm{Pb}$ collisions at $\sqrt{s_{\mathrm{NN}}}=30 \mathrm{TeV}$. Fig. 11 shows that the differential elliptic flows $v_{2}\left(p_{T}\right)$ have clear 


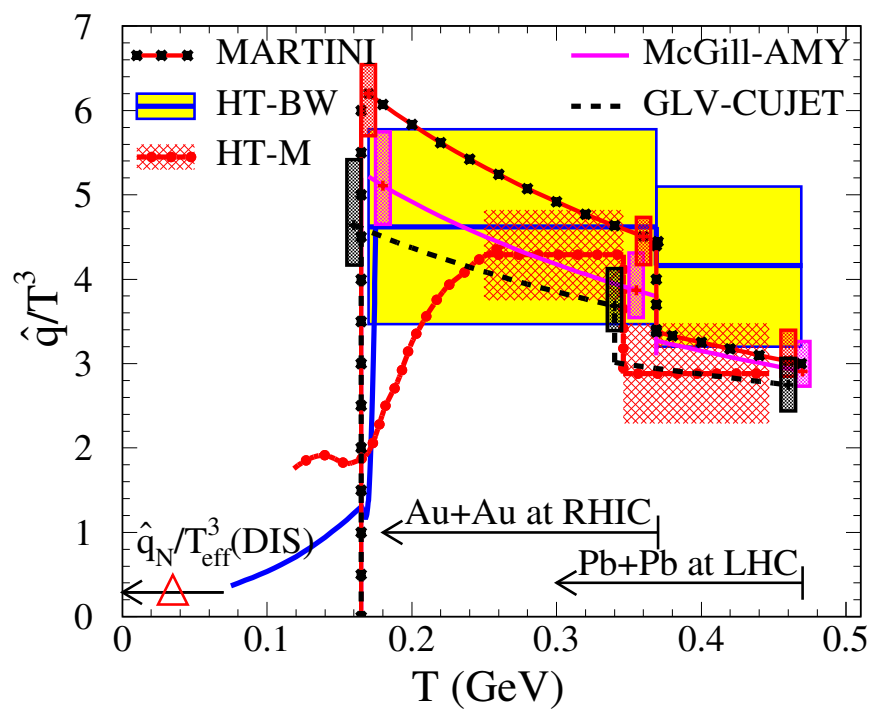

Figure 12: Scaled jet transport parameter $\hat{q} / T^{3}$ for an initial quark jet with energy $E=10 \mathrm{GeV}$ at the center of the most central $\mathrm{A}+\mathrm{A}$ collisions at an initial time $\tau_{0}=0.6 \mathrm{fm} / c$ constrained from recent analysis by the JET Collaboration [13] with $\chi^{2}$ fits to the suppression factors of single inclusive hadron spectra at RHIC and LHC. Errors from the fits are indicated by filled boxes at three separate temperatures at RHIC and LHC, respectively. The arrows indicate the range of temperatures at the center of the most central A+A collisions. The triangle indicates the value of $\hat{q}_{N} / T_{\text {eff }}^{3}$ in cold nuclei from DIS experiments.

mass ordering at different collision energies. As the collision energy increases from $2.76 \mathrm{TeV}$ to $30 \mathrm{TeV}$, the splitting of $v_{2}$ between pions and protons also increases due to the larger radial flow developed at higher collision energies. Meanwhile, Fig. 11 (middle and right panels) also shows that the elliptic flow of identified hadrons are sensitive to the QGP viscosity. Larger QGP shear viscosity leads to larger suppression of $v_{2}$. The measurement of elliptic flow of identified hadrons in the future heavy-ion collider will thus provide detailed information for the evolution of the QGP fireball, and help us to constrain the temperature dependence of the QGP shear viscosity.

\section{JET QUENCHING IN HEAVY-ION COLLISIONS}

In high-energy heavy-ion collisions, hard scattering of beam partons can produce energetic partons with very large transverse momentum. These energetic partons will fragment into large transverse momentum hadrons and appear in the detector as clusters of collimated hadrons which can be reconstructed as jets in experimental measurements. These initial energetic partons are produced in the very early stage of heavy-ion collisions and will certainly interact with soft partons from the bulk QGP that is formed over large volume of space. The interaction between jet partons and the QGP medium will lead to elastic and radiative energy loss and therefore suppression of the final state jets or large transverse momentum hadrons. These phenomena of jet quenching was originally proposed as one of the signatures of the QGP matter in high-energy heavy-ion collisions [128] which were first observed in heavy-ion collisions at RHIC [129]. After more than a decade of both theoretical and experimental studies at RHIC and LHC [10, jet quenching has become a powerful tool to study properties of the dense medium in heavy-ion collisions such as the jet transport parameter, defined as the broadening of averaged transverse momentum squared per unit length which is also related to the local gluon number density,

$$
\hat{q}=\frac{4 \pi^{2} \alpha_{s} C_{R}}{N_{c}^{2}-1} \int \frac{d y^{-}}{\pi}\left\langle F^{\sigma+}(0) F_{\sigma}^{+}(y)\right\rangle=\left.\frac{4 \pi^{2} \alpha_{s} C_{R}}{N_{c}^{2}-1} \rho_{A} x G_{N}(x)\right|_{x \rightarrow 0} .
$$




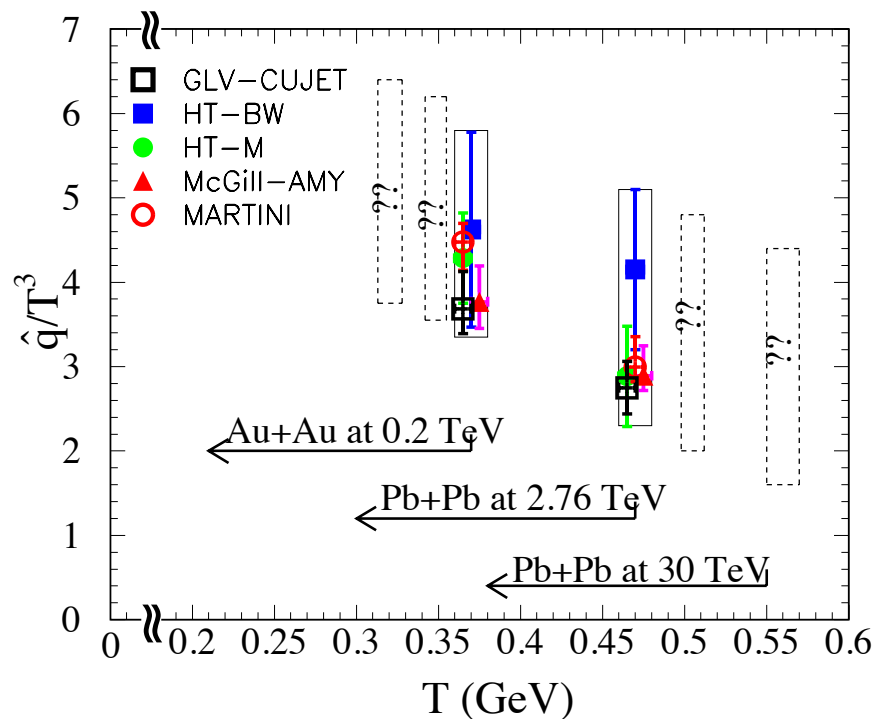

Figure 13: Scaled jet transport parameter $\hat{q} / T^{3}$ for an initial quark jet with energy $E=10 \mathrm{GeV}$ at the center of the most central $\mathrm{A}+\mathrm{A}$ collisions at an initial time $\tau_{0}=0.6 \mathrm{fm} / c$ constrained from recent analysis by the JET Collaboration 13. The dashed boxes indicate expected values in $\mathrm{A}+\mathrm{A}$ collisions at $\sqrt{s}=0.063,0.130,5.5$ and $30 \mathrm{TeV} / \mathrm{n}$, assuming the initial entropy is proportional to the final measured charged hadron rapidity density. The arrows indicate the range of temperatures at the center of the most central $\mathrm{A}+\mathrm{A}$ collisions at different colliding energies.

\section{A. Temperature dependence of jet transport parameter}

Since the hot bulk QGP medium is transient with a very short life-time and rapid expansion, the dynamical evolution of the bulk medium has to be taken into account for accurate descriptions of jet quenching phenomena. The hydrodynamical models as discussed in the previous section become necessary for jet quenching studies. One therefore needs a framework for the study that combines bulk medium evolution and jet quenching for extraction of jet transport parameter. A recent effort has been carried out by the JET topical collaboration to create a comprehensive Monte Carlo package which combines the most advanced model for bulk medium evolution, up-to-date models for parton propagation in medium and final hadronization of jet shower partons and jet-induced medium excitation. A comprehensive study has been carried out that surveyed five different approaches to parton energy loss combined with bulk medium evolution from $2+1 \mathrm{D}$ and $3+1 \mathrm{D}$ hydrodynamic models that have been constrained by the bulk hadron spectra [13]. Through $\chi^{2}$-fitting of the single inclusive hadron spectra at both RHIC and LHC with five different approaches to parton energy loss: GLV [130] and its recent CUJET implementation [131, the high-twist (HT) approaches (HT-BW and HT-M) [132, 133] and the MARTINI [134] and McGill-AMY [135] model, one obtained the most up-to-date constraints on the values of the jet transport parametersas shown in Fig. 12 13. Analyses of RHIC and LHC data with the YaJEM model [136] give similar constraints as shown in Fig. 12. The jet transport parameter extracted from these analyses are $\hat{q} \approx 1.2 \pm 0.3$ and $1.9 \pm 0.7 \mathrm{GeV}^{2} / \mathrm{fm}$ in the center of the most central $\mathrm{Au}+\mathrm{Au}$ collisions at $\sqrt{s}=200 \mathrm{GeV}$ and $\mathrm{Pb}+\mathrm{Pb}$ collisions at $\sqrt{s}=2.67 \mathrm{TeV}$, respectively, at an initial time $\tau_{0}=0.6$ $\mathrm{fm} / c$ for a quark jet with an initial energy of $10 \mathrm{GeV} / c$. When scaled by $T^{3}$, the natural scale in a QGP at high temperature, $\hat{q} / T^{3}$ represents the interaction strength between jets and the medium. Current values at RHIC and LHC indicate a possible gradual weakening toward higher colliding energies where the initial temperatures are also higher. At $\sqrt{s}=30 \mathrm{TeV}$, one expects to reach even higher initial temperatures in the center of $\mathrm{Pb}+\mathrm{Pb}$ collisions and further weakening of the jet-medium interaction. Shown in Fig. 13 as open boxes with question marks are the predicted values of $\hat{q}$ at this energy, higher LHC energy and lower energies of the beam energy scan program at RHIC. Together with the current values at the LHC and RHIC energy, one can provide a glimpse to the temperature dependence of $\hat{q} / T^{3}$. 


\section{B. Suppression of single hadron spectra}

For an estimate of the suppression of single inclusive hadron spectra in heavy-ion collisions at very high future collider energy, we use both the higher-twist (HT) [132] and McGill-AMY [135] model.

Within the HT approach, the effect of parton energy on the final hadron spectra is implemented through effective medium-modified fragmentation functions (FF) [137-139],

$$
\begin{aligned}
D_{h / c}\left(z_{c}, \Delta E_{c}, \mu^{2}\right)= & \left(1-e^{-\left\langle N_{g}^{c}\right\rangle}\right)\left[\frac{z_{c}^{\prime}}{z_{c}} D_{h / c}^{0}\left(z_{c}^{\prime}, \mu^{2}\right)\right. \\
& \left.+\left\langle N_{g}^{c}\right\rangle \frac{z_{g}^{\prime}}{z_{c}} D_{h / g}^{0}\left(z_{g}^{\prime}, \mu^{2}\right)\right]+e^{-\left\langle N_{g}^{c}\right\rangle} D_{h / c}^{0}\left(z_{c}, \mu^{2}\right),
\end{aligned}
$$

where $z_{c}^{\prime}=p_{T} /\left(p_{T c}-\Delta E_{c}\right), z_{g}^{\prime}=\langle L / \lambda\rangle p_{T} / \Delta E_{c}$ are the rescaled momentum fractions, $\Delta E_{c}$ is the radiative parton energy loss and $\left\langle N_{g}^{c}\right\rangle$ is the average number of induced gluon emissions. The FFs in vacuum $D_{h / c}^{0}\left(z_{c}, \mu^{2}\right)$ is given by the AKK08 parameterizations [140]. The total parton energy loss within the HT approach in a finite and expanding medium can be expressed as [141],

$$
\begin{aligned}
\frac{\Delta E_{a}}{E}= & C_{A} \frac{\alpha_{s}}{2 \pi} \int d y^{-} \int_{0}^{\mu^{2}} \frac{d l_{T}^{2}}{l_{T}^{4}} \int d z\left[1+(1-z)^{2}\right] \\
& \times \hat{q}_{a}(y) 4 \sin ^{2}\left[\frac{y^{-} l_{T}^{2}}{4 E z(1-z)}\right],
\end{aligned}
$$

in terms of the jet transport parameter $\hat{q}_{a}$ for a jet parton $a$. The jet transport parameter for a gluon is $9 / 4$ times of a quark and therefore the radiative energy loss of a gluon jet is also 9/4 times larger than that of a quark jet. According to the definition of jet transport parameter, we can assume that it is proportional to the local parton density in a QGP medium. In a dynamical evolving medium, it can be expressed in general as [132, 142, 143,

$$
\hat{q}(\tau, r)=\hat{q}_{0} \frac{\rho_{Q G P}(\tau, r)}{\rho_{Q G P}\left(\tau_{0}, 0\right)} \cdot \frac{p^{\mu} u_{\mu}}{p_{0}},
$$

In our calculation, we use a full $(3+1) \mathrm{D}$ ideal hydrodynamics [102, 144] to describe the space-time evolution of the local temperature and flow velocity in the bulk medium along the jet propagation path in heavy-ion collisions. Here $\rho_{Q G P}(\tau, r)$ is the parton density in the comoving frame of the fluid cell in hydrodynamics, and $\rho_{Q G P}\left(\tau_{0}, 0\right)$ is the initial parton density at the time $\tau_{0}=0.6 \mathrm{fm} / c$ in the center of the hot system, $p^{\mu}$ is the four momentum of the jet and $u^{\mu}$ is the four flow velocity in the collision frame, $\hat{q}_{0}$ denotes the jet transport parameter at the center of the bulk medium in the QGP phase at the initial time $\tau_{0}$.

The averaged number of gluon emissions $\left\langle N_{g}^{a}\right\rangle$ from the propagating parton $(a=q, g)$ within the high-twist approach of parton energy loss [145] is given by,

$$
\begin{aligned}
\left\langle N_{g}^{a}\left(\mu^{2}\right)\right\rangle= & C_{A} \frac{\alpha_{s}}{2 \pi} \int d y^{-} \int_{0}^{\mu^{2}} \frac{d l_{T}^{2}}{l_{T}^{4}} \int \frac{d z}{z}\left[1+(1-z)^{2}\right] \\
& \times \hat{q}_{a}(y) 4 \sin ^{2}\left[\frac{y^{-} l_{T}^{2}}{4 E z(1-z)}\right] .
\end{aligned}
$$

Using the above medium modified FFs with the collinear next-to-leading order (NLO) pQCD parton model [150, 151] and the CTEQ5 parameterization of parton distributions, one can calculate the final hadron spectra in both heavy-ion and $\mathrm{p}+\mathrm{p}$ collisions. Shown in Fig. 14 and Fig. 15 are the nuclear modification factors,

$$
R_{A A}=\frac{d \sigma_{A A} / d p_{T}^{2} d y}{\int d^{2} b T_{A A}(\mathbf{b}) d \sigma_{N N} / d p_{T}^{2} d y},
$$

for the charged hadron spectra as compared to the RHIC/LHC data on central collisions with different values for the jet transport parameter. The values of $\hat{q}$ from best $\chi^{2}$ fits are $\hat{q}_{0}=0.7-1.0 \mathrm{GeV}^{2} / \mathrm{fm}$ at RHIC energy and $\hat{q}_{0}=1.3-2.0 \mathrm{GeV}^{2} / \mathrm{fm}$ at LHC. This is consistent with HIJING 2.0 prediction [78] and the JET analyses [13].

Since the jet transport parameter $\hat{q}_{0}$ is proportional to the initial parton number density which in turn is proportional to the final charged hadron multiplicity, we can assume $\hat{q}_{0}=2.6-4.0 \mathrm{GeV}^{2} / \mathrm{fm}$ for a quark jet in central $\mathrm{Pb}+\mathrm{Pb}$ collisions at $\sqrt{s_{N N}}=30 \mathrm{TeV} / \mathrm{n}$ which is about 2 times that at LHC energy according to Table II Shown in Fig. 


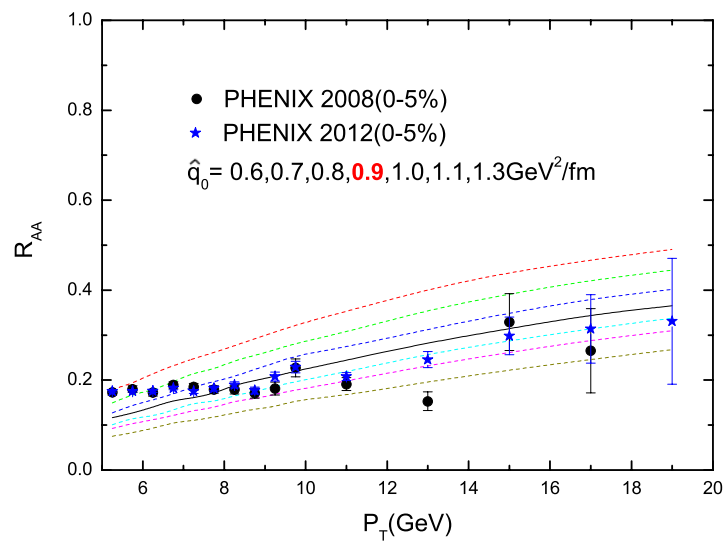

Figure 14: Nuclear modification factor at mid-rapidity for $\pi^{0}$ spectra in $0-5 \%$ central Au+Au collisions at $\sqrt{s_{N N}}=200 \mathrm{GeV}$ with a range of values of initial quark jet transport parameter $\hat{q}_{0}$ at $\tau_{0}=0.6 \mathrm{fm} / c$ in the center of the most central collisions (from top to bottom), as compared to PHENIX data [146, 147] at RHIC.

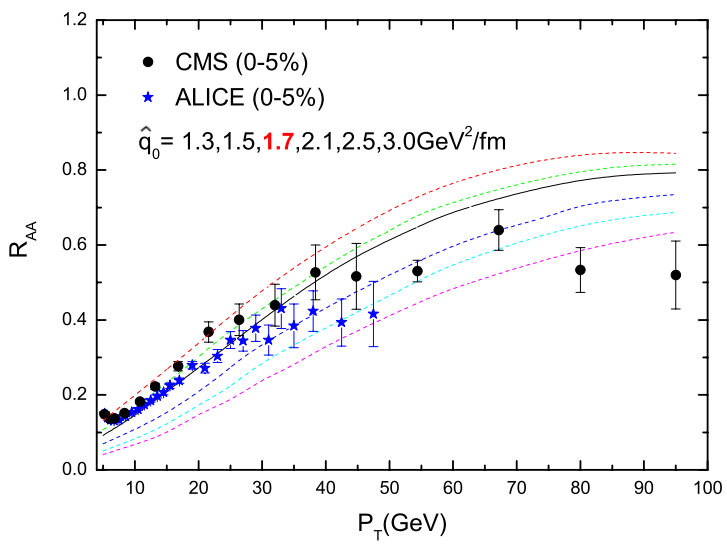

Figure 15: Nuclear modification factor at mid-rapidity for changed hadron spectra in $0-5 \%$ central $\mathrm{Pb}+\mathrm{Pb}$ collisions at $\sqrt{s_{N N}}=2.76 \mathrm{TeV} / \mathrm{n}$ with a range of values of initial quark jet transport parameter $\hat{q}_{0}$ at $\tau_{0}=0.6 \mathrm{fm} / c$ in the center of the most central collisions(from top to bottom), as compared to ALICE [148] and CMS data [149] at LHC.

16 is the nuclear modification factor at mid-rapidity for charged hadron spectra in $0-5 \%$ central $\mathrm{Pb}+\mathrm{Pb}$ collisions at $\sqrt{s_{N N}}=30.0 \mathrm{TeV} / \mathrm{n}$ with a range of values of initial quark jet transport parameter $\hat{q}_{0}=2.6-4.0 \mathrm{GeV}^{2} / \mathrm{fm}$ at $\tau_{0}=0.6 \mathrm{fm} / c$ in the center of the most central collisions(from top to bottom), as compared to $\mathrm{Pb}+\mathrm{Pb}$ collisions at $\sqrt{s_{N N}}=2.76 \mathrm{TeV} / \mathrm{n}$ with the value of $\hat{q}_{0}=1.3-2.0 \mathrm{GeV}^{2} / \mathrm{fm}$ and ALICE [148] and CMS data [149] at LHC as shown in Fig. 15. Over the range of $p_{T}=10-100 \mathrm{GeV} / c$, the hadron spectra are significantly more suppressed at $\sqrt{s}=30 \mathrm{TeV}$ than at LHC due to larger initial values of jet transport parameter. The difference becomes smaller at high transverse momentum due to different initial jet spectra at two different energies.

In the McGill-AMY approach [135, 152, nuclear modification of hadron spectra in nucleus-nucleus collisions can be calculated by first solving a set of coupled transport rate equations for the hard jet energy/momentum distributions $f(p, t)=d N(p, t) / d p$ in the hot nuclear medium. The coupled rate equations for quark and gluon jets may generically be written as the following form:

$$
\frac{d f_{j}(p, t)}{d t}=\sum_{a b} \int d k\left[f_{a}(p+k, t) \frac{d \Gamma_{a \rightarrow j}(p+k, k)}{d k d t}-P_{j}(k, t) \frac{d \Gamma_{j \rightarrow b}(p, k)}{d k d t}\right]
$$




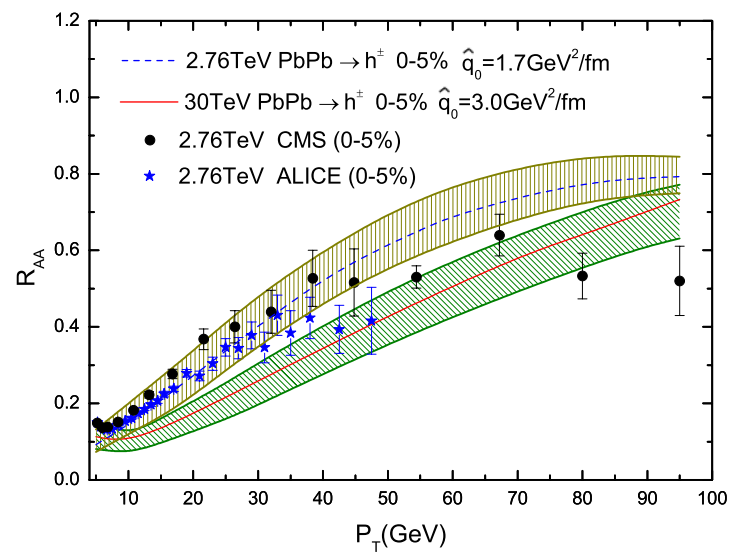

Figure 16: Nuclear modification factor at mid-rapidity for changed hadron spectra in $0-5 \%$ central $\mathrm{Pb}+\mathrm{Pb}$ collisions at $\sqrt{s_{N N}}=30.0 \mathrm{TeV} / \mathrm{n}$ with a range of values of initial quark jet transport parameter $\hat{q}_{0}=2.6-4.0 \mathrm{GeV} / \mathrm{fm}$ at $\tau_{0}=0.6 \mathrm{fm} / c$ in the center of the most central collisions(from top to bottom), as compared to $\mathrm{Pb}+\mathrm{Pb}$ collisions at $\sqrt{s_{N N}}=2.76 \mathrm{TeV} / \mathrm{n}$ with the value of $\hat{q}_{0}$ is from $1.3 \mathrm{GeV}^{2} / \mathrm{fm}$ to $2.0 \mathrm{GeV}^{2} / \mathrm{fm}$ and ALICE [148] and CMS data [149] at LHC as shown in Fig. 15]

In the above equation, $d \Gamma_{j \rightarrow a}(p, k) / d k d t$ represents the transition rate for the process $j \rightarrow a$, with $p$ the initial jet energy and $k$ the momentum lost in the process. The transition rates for radiative processes are taken from Refs. [153 155], and for the collisional processes, the drag and the diffusion contributions are incorporated following Refs. [135, 152. The contributions from energy gain processes are taken into account by the $k<0$ integral.

After solving the above coupled rate equations, one may obtain the medium-modified fragmentation function as follows:

$$
\tilde{D}_{h / j}\left(z, \vec{r}_{\perp}, \phi_{p}\right)=\sum_{j^{\prime}} \int d p_{j^{\prime}} \frac{z^{\prime}}{z} D_{h / j^{\prime}}\left(z^{\prime}\right) P\left(p_{j^{\prime}} \mid p_{j}, \vec{r}_{\perp}, \phi_{p}\right)
$$

where $z=p_{h} / p_{j}$ and $z^{\prime}=p_{h} / p_{j^{\prime}}$, with $p_{h}$ the momentum of the hadron $h$ and $p_{j}\left(p_{j^{\prime}}\right)$ the initial (final) jet momentum. $D_{h / j}(z)$ is the vacuum fragmentation function, and $P\left(p_{j^{\prime}} \mid p_{j}, \vec{r}_{\perp}, \phi_{p}\right)$ represents the differential probability for obtaining a parton $j^{\prime}$ with momentum $p_{j^{\prime}}$ from a given parton $j$ with momentum $p_{j}$. This probability distribution depends on the path traveled by the parton and the local medium profiles such as the temperature and flow along that path. Therefore, $P\left(p_{j^{\prime}} \mid p_{j}, \vec{r}_{\perp}, \phi_{p}\right)$ depends on the the initial jet production location $\vec{r}_{\perp}$ and the propagation direction $\phi_{p}$. Jets are decoupled from the medium when the local temperature of the nuclear medium is below the transition temperature $T_{c}=160 \mathrm{MeV}$.

By convoluting the medium-modified fragmentation function with the initial jet momentum distribution computed from perturbative $\mathrm{QCD}$, one may obtain the final medium-modified hadron spectra:

$$
\frac{d \sigma_{A B \rightarrow h X}}{d^{2} p_{T}^{h} d y}=\int d^{2} \vec{r}_{\perp} \mathcal{P}_{A B}\left(\vec{r}_{\perp}\right) \sum_{j} \int \frac{d z}{z^{2}} \tilde{D}_{h / j}\left(z, \vec{r}_{\perp}, \phi_{p}\right) \frac{d \sigma_{A B \rightarrow j X}}{d^{2} p_{T}^{j} d y} .
$$

In the above equation, $\mathcal{P}_{A B}\left(b, \vec{r}_{\perp}\right)$ is the probability distribution of the initial jet production position $\vec{r}_{\perp}$, and is determined from binary collision distribution simulated by the Glauber model. One may fix the propagation direction $\phi_{p}$ or average over a certain range.

Putting the above ingredients together, one may obtain the hadron yield after medium modification and calculate the nuclear modification factor $R_{A A}$.

In Fig. 17, we show the comparison of the calculated nuclear modification factor $R_{A A}$ from McGill-AMY approach as a function of $p_{T}$ for: central $0-5 \% \mathrm{Au}-\mathrm{Au}$ collisions at $200 \mathrm{GeV} / \mathrm{n}$ at $\mathrm{RHIC}$, central $0-5 \% \mathrm{~Pb}-\mathrm{Pb}$ collisions at $2.76 \mathrm{TeV} / \mathrm{n}$ at the LHC, and central $0-5 \% \mathrm{~Pb}-\mathrm{Pb}$ collisions at $30 \mathrm{TeV} / \mathrm{n}$. Note that in the McGill-AMY model, the model parameter is the strong coupling constant $\alpha_{s}$ which is usually fitted to the experimental data. For RHIC $\mathrm{Au}-\mathrm{Au}$ collisions it is obtained as $\alpha_{s}=0.25$ by fitting to PHENIX data, and for the LHC $\alpha_{s}=0.23$ using CMS and ALICE data. The decreasing of $\alpha_{s}$ from RHIC to the LHC may be understood as originating from the increasing of the average temperature (or the energy density) of the hot nuclear media produced at RHIC and LHC. To account 


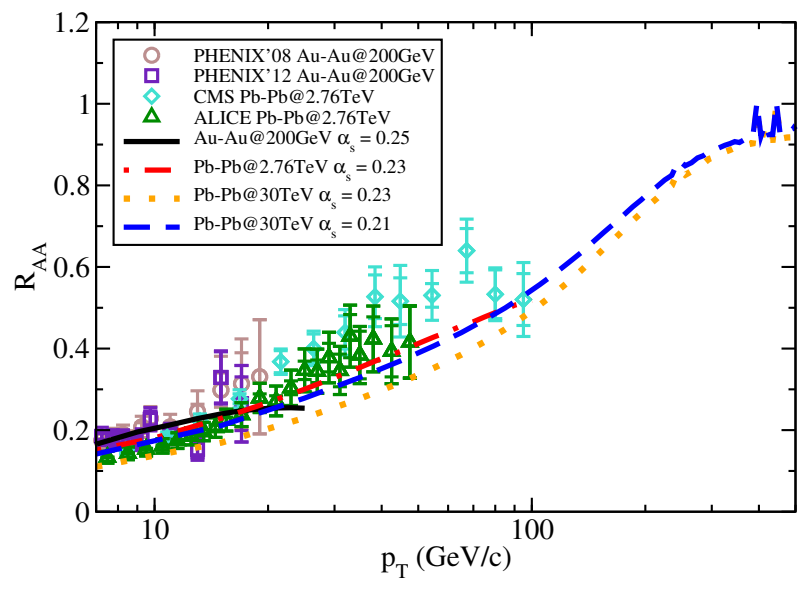

Figure 17: (Color online) The nuclear modification factor $R_{A A}$ as a function of $p_{T}$ for central $\mathrm{Au}-\mathrm{Au}$ collisions at $200 \mathrm{GeV} / \mathrm{n}$ at RHIC, for central $\mathrm{Pb}-\mathrm{Pb}$ collisions at $2.76 \mathrm{TeV} / \mathrm{n}$ at the $\mathrm{LHC}$, and for central $\mathrm{Pb}-\mathrm{Pb}$ collisions at $30 \mathrm{TeV} / \mathrm{n}$.

for such effect when moving from $2.76 \mathrm{TeV} / \mathrm{n} \mathrm{Pb}-\mathrm{Pb}$ collisions to $30 \mathrm{TeV} / \mathrm{n} \mathrm{Pb}-\mathrm{Pb}$ collisions, we decrease the strong coupling constant from $\alpha_{s}=0.23$ to $\alpha_{s}=0.21$. One may consider the calculation for $30 \mathrm{TeV} / \mathrm{n} \mathrm{Pb}-\mathrm{Pb}$ collisions using $\alpha_{s}=0.23$ as the lower reference bound for the nuclear modification factor $R_{A A}$.

\section{Medium modification of reconstructed jets}

The jet quenching or parton energy loss in hot and dense QGP can affect not only hadron suppression but also in jet modifications in high-energy nuclear collisions [156, 157. The study of fully reconstructed jet production in relativistic heavy-ion collisions plays a very important role in probing the properties of the QGP formed in $\mathrm{Pb}+\mathrm{Pb}$ reactions at the LHC [158. Full jets in experiments are reconstructed from hadronic energies measured either through tracking or calorimetric detectors or both with a given jet -inding algorithm [159]. The jet production cross section with the same jet-finding algorithm can also be calculated within the next-to-leading (NLO ) pQCD, using Monte Carlo packages such as MEKS [160]. Inclusive differential jet production cross sections in $\mathrm{p}+\mathrm{p}$ collisions at NLO accuracy provide the baseline to calculate inclusive jet productions in heavy-ion collisions [157],

$$
\frac{1}{\left\langle N_{\mathrm{bin}}\right\rangle} \frac{d \sigma^{A A}(R)}{d y d E_{T}}=\int_{\epsilon=0}^{1} d \epsilon \sum_{q, g} P_{q, g}(\epsilon) \frac{1}{1-\left(1-f_{q, g}\right) \cdot \epsilon} \frac{d \sigma_{q, g}^{p p}(C N M)}{d y d E_{T}^{\prime}} .
$$

In the above expression for jet production cross section in heavy-ion collisions, several cold nuclear matter effects (shadowing, anti-shadowing and EMC effect) are taken into account through the EPS09 [161] parameterization of nuclear parton distribution functions (nPDF). The parameter $f_{q, g}$ is the part of the fractional energy loss falling in the jet area, and can be calculated from the angular distribution of medium induced parton energy loss [157, 162], $P_{q, g}(\epsilon)$ is the probability that a jet loses energy fraction $\epsilon E_{T}^{\prime}$, here $E_{T}^{\prime}=E_{T} /\left[1-\left(1-f_{q, g}\right) \cdot \epsilon\right][157$.

Shown in Fig. 18 are the nuclear modification factors of inclusive jet production,

$$
R_{A A}^{\mathrm{jet}}=\frac{d \sigma^{A A} / d y d E_{T}}{\left\langle N_{\mathrm{bin}}\right\rangle d \sigma^{p p} / d y d E_{T}}
$$

in central $\mathrm{Pb}+\mathrm{Pb}$ collisions for different jet radius $R=0.3,0.4,0.5$ at $\sqrt{s_{N N}}=20 \mathrm{TeV}$. One-dimension longitudinal Bjorken expansion of the QGP fireball with Glauber transverse distribution and a highest initial temperature $T_{0}=570$ $\mathrm{MeV}$ is assumed. The calculated jet suppression factors increase with jet transverse energy for all three different jet radii. The suppression factor for a larger jet cone size is slightly less because more radiated gluon remain inside the jet cone and thus less effective energy loss for the reconstructed jet. These calculated suppression factors for reconstructed jets at $\sqrt{s}=20 \mathrm{TeV}$ are somewhat similar to that measured at the current LHC energy [163, 164] even though the initial parton energy density or the jet transport parameter is almost a factor of 2 larger. This indicates that the jet suppression factor is less sensitive to the properties of the medium as compared to the single inclusive hadrons. It is therefore helpful to explore other observables such as jet shape or profile functions. 


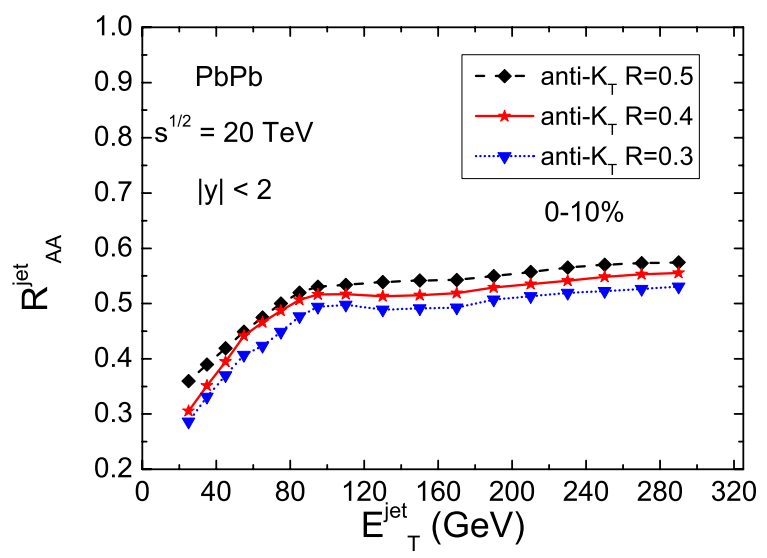

Figure 18: Nuclear modification factor $R_{A A}$ for inclusive jet production as a function of jet transverse energy for different jet radius in central $\mathrm{Pb}+\mathrm{Pb}$ collisions at $\sqrt{s_{N N}}=20 \mathrm{TeV}$.

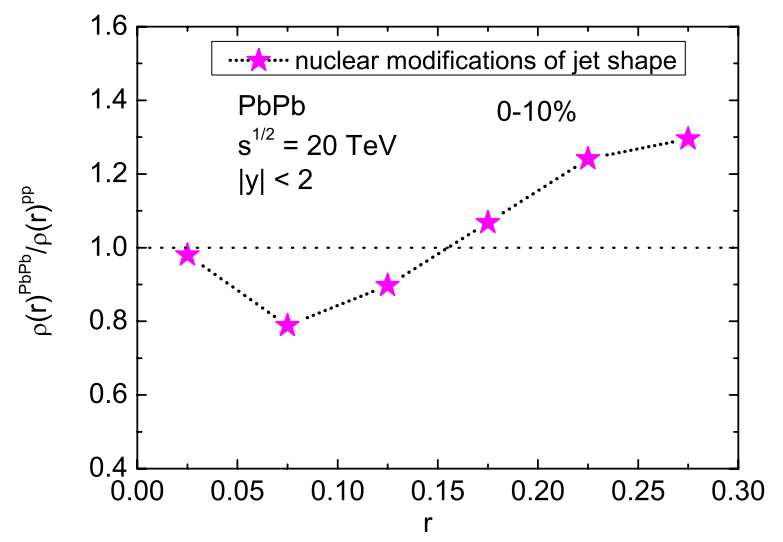

Figure 19: Nuclear modification $R_{A A}^{\text {jet-shape }}$ for differential jet shapes with $R=0.3$ in central $\mathrm{Pb}+\mathrm{Pb}$ collisions at $\sqrt{s}=20$ TeV .

Jet shape, also called jet energy profile, is the internal energy distribution of a jet. Medium modification of the jet shape in heavy-ion collisions due to multiple scattering and induced radiation relative to hadron-hadron reactions has shown to be sensitive to jet-medium interaction [156, 157]. The differential jet shape is defined as

$$
\rho(r)=\frac{1}{\Delta r} \frac{1}{N^{\text {jet }}} \sum_{\text {jets }} \frac{P_{T}(r-\Delta r / 2, r+\Delta r / 2)}{P_{T}(0, R)}, \quad \Delta r / 2 \leq r \leq R-\Delta r / 2 .
$$

Jet shapes in hadronic collisions have been studied recently in the framework of QCD resummation at NLO 165, 166, which give a decent description of jet profiles in $\mathrm{p}+\mathrm{p}$ collisions and provide the baseline for investigating jet shape modification in high-energy nuclear colliisons. In heavy-ion reactions, the jet energy consists of two parts: the energy of quenched leading parton $\left(E_{p}\right)$ and the redistributed energy of radiated gluon $\left(E_{g}\right)$ inside the jet cone. The total jet energy should be their sum, $E^{\text {jet }}=E^{g}+E^{p}$. Thus jet shapes in heavy-ion collisions can be calculated as follows

$$
\rho^{A A}\left(r, E_{\text {jet }}\right)=\frac{E_{g}}{E_{\text {jet }}} \rho^{\text {medium }}\left(r, E_{g}\right)+\frac{E_{p}}{E_{\text {jet }}} \rho^{p p}\left(r, E_{p}\right),
$$

where $\rho^{\text {medium }}\left(r, E_{g}\right)$ is calculated from the angular spectra of medium induced gluon radiation. Furthermore we 
define the nuclear modification ratio of jet shapes as

$$
R_{A A}^{\text {jet-shape }}=\frac{\rho^{A A}\left(r, E_{\mathrm{jet}}\right)}{\rho^{p p}\left(r, E_{\mathrm{jet}}\right)} .
$$

Shown in Fig. 19 is the calculated the nuclear modifications of jet shapes in central $\mathrm{Pb}+\mathrm{Pb}$ collisions at $\sqrt{s}=20 \mathrm{TeV}$. One can see a considerable enhancement of jet shapes in heavy-ion collisions relative to those in $\mathrm{p}+\mathrm{p}$ in the region when $r \rightarrow R$ due to induced gluon radiation while there is some depletion of jet energy distribution at intermediate $r$ due to fixed total jet energy. Such a feature has been observed in heavy-ion collisions at LHC [167] and should provide information on jet-medium interaction at future high-energy heavy-ion colliders.

\section{MEDIUM MODIFICATION OF OPEN HEAVY MESONS}

An alternative candidate of hard probe of the QGP properties is heavy flavor meson. Since the large mass of heavy quarks effectively suppresses their thermal production from the bulk matter, the majority of them are produced at the primordial stage of nuclear collisions through hard scatterings. After that, they propagate through the hot QGP matter with their flavor conserved and therefore serve as a clean probe of the whole evolution history of the QGP fireballs.

\section{A. Perturbative heavy quark transport}

In this section, we adopt an improved Langevin approach [168, 169 to simulate the in-medium energy loss of open heavy quarks. The hadronization into heavy mesons is simulated using a hybrid model of fragmentation and coalescence developed in Ref. [169].

In the limit of small momentum transfer in each interaction, multiple scatterings of heavy quarks inside a thermalized medium can be described using the Langevin equation. Apart from the collisional energy loss due to these quasi-elastic scatterings, heavy quarks may also lose energy via medium-induced gluon radiation. We modify the classical Langevin equation as follows to simultaneously incorporate these two energy loss mechanisms:

$$
\frac{d \vec{p}}{d t}=-\eta_{D}(p) \vec{p}+\vec{\xi}+\vec{f}_{g}
$$

The first two terms on the right-hand side of Eq.15) are inherited from the original Langevin equation, describing the drag force and thermal random force exerted on heavy quarks when they scatter with light partons in the medium background. We assume that the fluctuation-dissipation theorem is still hold between these two terms $\eta_{D}(p)=\kappa /(2 T E)$, in which $\kappa$ is known as the momentum-space diffusion coefficient of heavy quarks: $\left\langle\xi^{i}(t) \xi^{j}\left(t^{\prime}\right)\right\rangle=$ $\kappa \delta^{i j} \delta\left(t-t^{\prime}\right)$. The third term $\vec{f}_{g}$ in Eq. $\sqrt{15}$ is introduced to describe the recoil force heavy quarks experience when they radiate gluons. The probability of gluon radiation within the time interval $[t, t+\Delta t]$ can be evaluated according to the number of radiated gluons,

$$
P_{\mathrm{rad}}(t, \Delta t)=\left\langle N_{\mathrm{g}}(t, \Delta t)\right\rangle=\Delta t \int d x d k_{\perp}^{2} \frac{d N_{\mathrm{g}}}{d x d k_{\perp}^{2} d t},
$$

as long as $\Delta t$ is chosen sufficiently small so that $P_{\text {rad }}(t, \Delta t)<1$ is always satisfied. In our study, the distribution of the medium-induced gluon radiation is taken from the high-twist approach to parton energy loss [170 172]:

$$
\frac{d N_{\mathrm{g}}}{d x d k_{\perp}^{2} d t}=\frac{2 C_{A} \alpha_{s} P(x) \hat{q}}{\pi k_{\perp}^{4}} \sin ^{2}\left(\frac{t-t_{i}}{2 \tau_{f}}\right)\left(\frac{k_{\perp}^{2}}{k_{\perp}^{2}+x^{2} M^{2}}\right)^{4},
$$

where $x$ is the fractional energy taken from the heavy quark by the radiated gluon, $k_{\perp}$ is the gluon transverse momentum, $P(x)$ is the quark splitting function and $\tau_{f}=2 E x(1-x) /\left(k_{\perp}^{2}+x^{2} M^{2}\right)$ is the gluon formation time. In Eq. (17), a quark transport coefficient $\hat{q}$ is utilized, which is related to the diffusion coefficient $\kappa$ by adding the factor of dimension in our work $\hat{q}=2 \kappa$. With this assumption, only one free parameter remains in the modified Langevin equation [Eq. 15]]. As shown in the earlier work [169, $\hat{q} / T^{3}=5.0$ is chosen to best describe the experimental data of heavy flavor meson at high $p_{\mathrm{T}}$ at RHIC and LHC.

With this improved Langevin approach, we may simulate the evolution of heavy quarks in relativistic nuclear collisions. The dense QCD medium produced in these collisions is simulated with a $(3+1)$-dimensional ideal hydrodynamic 


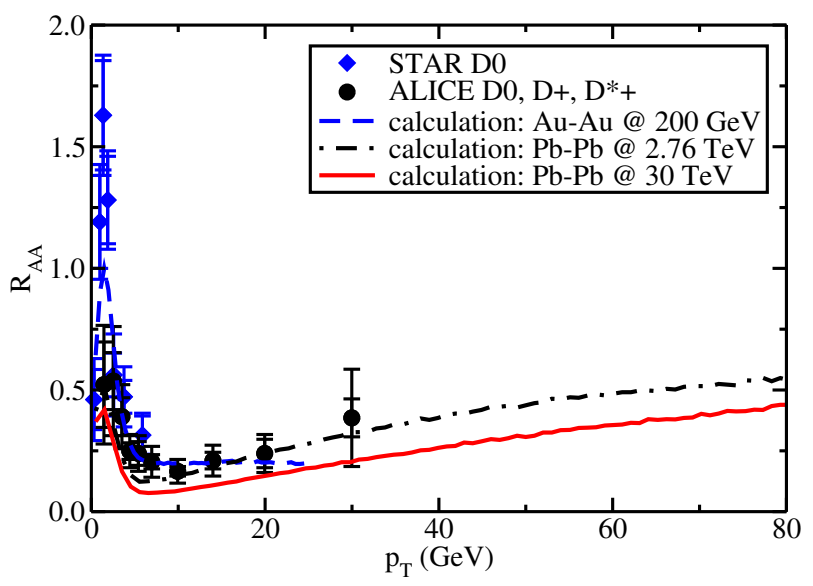

Figure 20: (Color online) The nuclear modification factor $R_{\mathrm{AA}}$ of $D$ mesons, compared between in central Au-Au collisions at $200 \mathrm{AGeV}$, in central $\mathrm{Pb}-\mathrm{Pb}$ collisions at 2.76 ATeV, and in central $\mathrm{Pb}-\mathrm{Pb}$ collisions at $30 \mathrm{ATeV}$.

model 102, 144]. This hydrodynamic calculation provide the space-time evolution of the local temperature and fluid velocity of the QGP fireballs. With these information, for every time step, we boost each heavy quark into the local rest frame of the fluid cell through which it propagates and then update its energy and momentum according to our modified Langevin equation. After that, the heavy quark is boosted back to the global center of mass frame and stream freely until its interaction with the medium for next time step. Before the hydrodynamical evolution commences (at $\tau_{0}=0.6 \mathrm{fm} / \mathrm{c}$ ), heavy quarks are initialized with a MC-Glauber model for their spatial distribution and a leading order pQCD calculation together with the CTEQ5 parton distribution functions [173] for their momentum distribution. The nuclear shadowing effect in the initial state of nucleus-nucleus collisions is taken into account by using the EPS09 parameterization [161. With these initializations, heavy quarks evolve inside the QGP matter until they reach fluid cells with local temperature below $T_{\mathrm{c}}(165 \mathrm{MeV})$. Then they hadronize into heavy flavor hadrons within a hybrid model of fragmentation and coalescence. The relative probability of fragmentation and heavy-light quark coalescence is calculated with the Wigner functions in an instantaneous coalescence model [174]. If a heavy quark is chosen to combine with a thermal parton from the medium, the momentum distribution of the produced hadron is calculated directly with the coalescence model itself. On the other hand, if the heavy quark is chosen to fragment, its fragmentation process is simulated with PYTHIA 6 [175] in which the Peterson fragmentation function is used.

In Fig 20, the suppression factors for $D$ meson $R_{\mathrm{AA}}$ are shown for different collisional energies. For central Au$\mathrm{Au}$ collisions at $200 \mathrm{AGeV}$, a bump structure in the $D$ meson $R_{\mathrm{AA}}$ can be observed around 1-2 GeV. This is mainly contributed by the coalescence mechanism in heavy quark hadronization process, which combines low $p_{\mathrm{T}}$ heavy quarks and light thermal partons and enhances the production of $D$ mesons at medium $p_{\mathrm{T}}$. Such a bump is significantly suppressed in $\mathrm{Pb}-\mathrm{Pb}$ collisions at $2.76 \mathrm{ATeV}$ and $30 \mathrm{ATeV}$ due to the strong nuclear shadowing effect for these collisional energies at low $p_{\mathrm{T}}$. At higher $p_{\mathrm{T}}$, the $D$ meson $R_{\mathrm{AA}}$ is relatively flat between 10 and $20 \mathrm{GeV}$ in $\mathrm{Au}-\mathrm{Au}$ collisions at $200 \mathrm{AGeV}$, but starts to increase with $p_{\mathrm{T}}$ in 2.76 and $30 \mathrm{ATeV} \mathrm{Pb}-\mathrm{Pb}$ collisions. This probably results from the harder initial $p_{\mathrm{T}}$ spectra of charm quarks produced at the LHC energy than at the RHIC energies. In addition, we observe that $D$ mesons are more suppressed in $30 \mathrm{ATeV}$ than in $2.76 \mathrm{ATeV} \mathrm{Pb}-\mathrm{Pb}$ collisions, since larger collisional energy leads to higher initial temperature and longer lifetime of the QGP fireballs and therefore increases the total in-medium energy loss of heavy quarks.

\section{B. Non-perturbative heavy quark transport}

A non-perturbative transport model for heavy quarks and open heavy-flavor (HF) mesons in ultrarelativistic heavyion collisions was introduced in Ref. [176. The HF transport is simulated with relativistic Langevin simulations with temperature and momentum dependent transport coefficients computed from $T$-matrix interactions as described below. It treats both microscopic HF transport through quark-gluon plasma (QGP) and hadronization in a strongcoupling scheme. Its applications to HF phenomenology at RHIC [177, 178, and LHC [179] result in fair agreement with existing data for the nuclear modification factor and elliptic flow of $D$ mesons, $B$ mesons (from non-prompt $J / \psi$ ) and HF decay leptons.

In the deconfined high-temperature phase, heavy-quark (HQ) scattering with medium partons is calculated using 
the thermodynamic $T$-matrix approach [180, 181, which accounts for all color channels (e.g., $a=1,8$ for $Q \bar{q})$, different flavors $(u, d, s$ and gluons) and partial waves $(l=S, P)$ via a Lippmann-Schwinger equation of the type

$$
T_{l, a}=V_{l, a}+\frac{2}{\pi} \int_{0}^{\infty} k^{2} d k V_{l, a} G_{2} T_{l, a} ;
$$

here, $G_{2}$ is the uncorrelated in-medium two-particle propagator which includes the single-quark self-energies. The potential, $V_{l, a}$, the kernel of the integral equation, is approximated by the internal energy computed in thermal latticeQCD (1QCD) [182, 183, and incorporates relativistic corrections to recover the correct high-energy perturbative limit. The use of the internal energy yields better agreement with IQCD data on, e.g., quarkonium correlators, HQ susceptibilities and the HQ diffusion coefficient, than the free energy [184]. As the color-screening of the potential gradually reduces when approaching the critical temperature, $T_{\mathrm{pc}} \simeq 170 \mathrm{MeV}$, from above, the remnant confining potential strengthens and the HQ interactions with light quarks in the QGP start to develop heavy-light $(D$ - of $B$-meson like) resonance correlations.

Once the medium evolution reaches the critical region, the resonant $Q \bar{q}$ correlations emerging from the $T$-matrices are utilized as a hadronization mechanism via the resonance recombination model (RRM) [185] on a hydrodynamic hypersurface at $T_{\mathrm{pc}}$; left-over charm and bottom quarks are hadronized via FONLL fragmentation [179] as used for the initial spectra in $p p$ (for which EPS09 shadowing has been accounted for [179]). The $D$ and $B$ mesons thus formed continue to diffuse in the subsequent hadronic phase via scatterings off bulk hadrons $\left(\pi, K, \eta, \rho, \omega, K^{*}, N, \bar{N}, \Delta\right.$ and $\bar{\Delta}$ ), evaluated using effective hadronic lagrangians available from the literature [186]. Around $T_{\mathrm{pc}}$ the resulting diffusion coefficient for $D$ mesons turns out to be comparable to the $T$-matrix results for charm quarks on the partonic side.

The hydrodynamic evolution utilizes the 2+1D ideal hydro code AZHYDRO [187, augmented with a modern lQCD equation of state for the QGP which is matched in a near-smooth transition to a hadron resonance gas (HRG) at $T_{\mathrm{pc}}=170 \mathrm{MeV}$. The chemical freezeout of hadrons is implemented for temperatures below $T_{\mathrm{ch}}=160 \mathrm{MeV}$, utilizing effective chemical potentials for hadrons stable under strong interactions. Our hydro tune in $\mathrm{Pb}+\mathrm{Pb}$ collisions consists of initial conditions from a Glauber model with an initialization time of $0.4 \mathrm{fm} / c$ (without initial flow nor fluctuations), which allows for a reasonable description of the bulk-hadron spectra and inclusive elliptic flow at kinetic freezeout at $2.76 \mathrm{TeV}$ [179]. It features a fast build-up of radial flow as well as bulk momentum anisotropy. As a result, the bulk $v_{2}$ gets almost saturated around $T_{c}$, which helps to develop substantial elliptic flow for both heavy quarks and thermal electromagnetic emissions (dileptons and photons).

In Fig. 21 we summarize our predictions for the nuclear modification factor $\left(R_{\mathrm{AA}}\right)$ and elliptic flow $\left(v_{2}\right)$ of charm quarks and $D$ mesons in $30 \mathrm{TeV} \mathrm{Pb}+\mathrm{Pb}$ collisions; the corresponding results for bottom quarks and $B$ mesons are shown in Fig. 22. The results overall are similar to $\mathrm{Pb}+\mathrm{Pb}$ collisions at the current LHC energy. A careful examination of the results shows, however, that the peak of $R_{A A}$ at low $p_{T}$ due to diffusion and parton recombination for hadronization shifts to higher $p_{T}$ because of the higher temperature and radial flow achieved at the higher colliding energy. The predicted suppression factors are also larger than that given in the perturbative approach. This may be caused by the lack of radiative energy loss in this non-perturbative approach which is important at high $p_{T}$. In this model calculation, heavy quark diffusion in QGP contributes to about $60-70 \%$ of the final total $v_{2}$ of $D / B$ mesons. The remaining contribution is due to coalescence of heavy and light quarks during hadronization and interaction of $D / B$ mesons during the hadronic phase. Therefore, interactions of heavy flavor with medium during the entire evolution of the medium are all indispensable for $D / B$ mesons to develop large final $v_{2}$ that could reach as much as $12-14 \%$ (for $D$ mesons) and 6-8\% (for $B$ mesons) in semi-central collisions.

\section{VI. $J / \psi$ PRODUCTION}

The suppression of $J / \psi$ in hot medium has been considered as a probe of the QGP created in the early stage of heavy ion collisions [188]. The nuclear modification factor $R_{A A} \sim 0.3$ in central $\mathrm{Au}+\mathrm{Au}$ collisions at RHIC [189] goes up to about 0.5 in central $\mathrm{Pb}+\mathrm{Pb}$ collisions at $\sqrt{s_{N N}}=2.76 \mathrm{TeV}$ at LHC [190] due to the increasing contribution of charmonium regeneration [191-193]. One can similarly investigate the behavior of nuclear modification factor $R_{A A}$ of $J / \psi$ at tens of $\mathrm{TeV}$, e.g. $\sqrt{s_{N N}}=20 \mathrm{TeV}$ in the framework of transport approach [194 197.

Considering that charmonium is so heavy and difficult to be thermalized in heavy-ion collisions, one can use the classical transport equation to describe the charmonium motion in the medium,

$$
\frac{\partial f_{\Psi}}{\partial t}+\frac{\mathbf{p}}{E_{\Psi}} \cdot \nabla f_{\Psi}=-\alpha_{\Psi} f_{\Psi}+\beta_{\Psi},
$$

where $f_{\Psi}(\mathbf{x}, \mathbf{p}, t)$ are the charmonium distribution functions in phase space for $\Psi=J / \psi, \psi^{\prime}, \chi_{c}$. Considering the fact that $J / \psi$ 's in $\mathrm{p}+\mathrm{p}$ collisions come from direct production and decay from excited states $\psi^{\prime}$ and $\chi_{c}$, one needs 

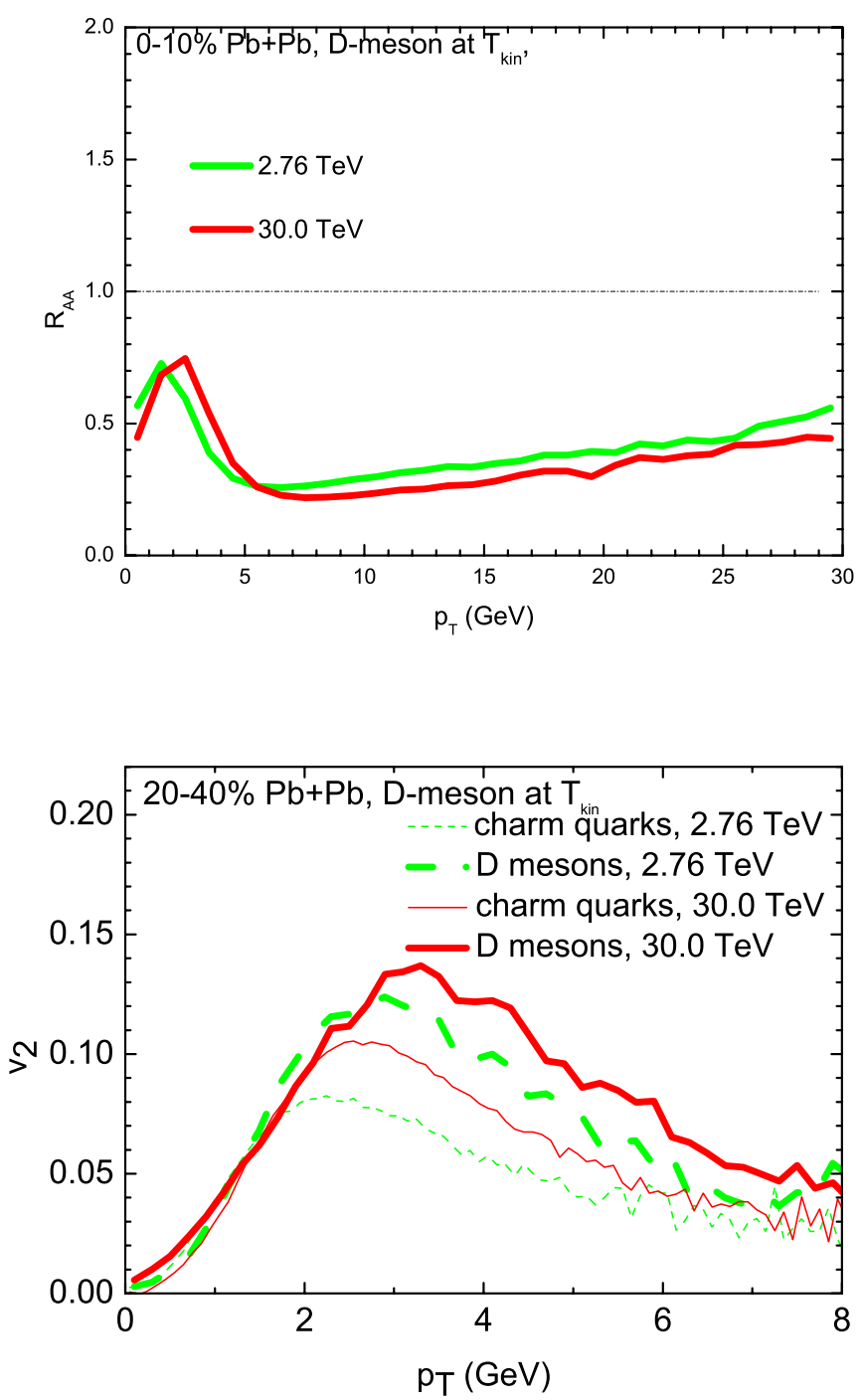

Figure 21: (Color online) The $R_{\mathrm{AA}}$ (upper panel) and $v_{2}$ (lower panel) of charm quarks and $D$ mesons for semi-central $\mathrm{Pb}+\mathrm{Pb}$ collisions at $\sqrt{s_{N N}}=2.76 \mathrm{TeV}$ and $30 \mathrm{TeV}$.

the distributions of $\psi^{\prime}$ and $\chi_{c}$. The charmonium energy is denoted by $E_{\Psi}=\sqrt{m_{\Psi}^{2}+\mathbf{p}^{2}}$, and $\alpha_{\Psi}$ and $\beta_{\Psi}$ are the charmonium dissociation and regeneration rates. Taking the gluon dissociation $g+\Psi \rightarrow c+\bar{c}$ as the main dissociation process at high temperature, the loss term $\alpha_{\Psi}$ can be expressed as,

$$
\begin{aligned}
\alpha_{\Psi}(\mathbf{x}, \mathbf{p}, t \mid \mathbf{b})= & \frac{1}{2 E_{\Psi}} \int \frac{d^{3} \mathbf{k}}{(2 \pi)^{3}} \frac{1}{2 E_{g}} W_{g \Psi}^{c \bar{c}}(\mathbf{p}, \mathbf{k}) f_{g}(\mathbf{x}, \mathbf{k}, t) \\
& \times \Theta\left(T(\mathbf{x}, t \mid \mathbf{b})-T_{c}\right),
\end{aligned}
$$

with impact parameter $\mathbf{b}$ describing the centrality of collisions, gluon energy $E_{g}$, the thermal gluon distribution $f_{g}$ and the dissociation probability $W_{g \Psi}^{c \bar{c}}$. The dissociation probability is determined by the dissociation cross section from gluons whose vacuum value $\sigma_{\Psi}(0)$ is calculated through the operator production expansion [198, 199]. Temperature dependent cross section $\sigma_{\Psi}(T)$ can be estimated by taking into account the geometry relationship between the cross section and the size of $J / \psi, \sigma_{\Psi}(T)=\sigma_{\Psi}(0)\left\langle r_{\Psi}^{2}\right\rangle(T) /\left\langle r_{\Psi}^{2}\right\rangle(0)$. The charmonium size $\left\langle r_{\Psi}^{2}\right\rangle(T)$ can be calculated in the potential model [200]. The step function $\Theta$ means that we considered here only the dissociation (and regeneration) in the deconfined phase with $T_{c}$ being the critical temperature for the deconfinement phase transition. Considering the strong interaction between charm quarks and the high-temperature medium at colliding energy $\sqrt{s_{N N}}=20$ 

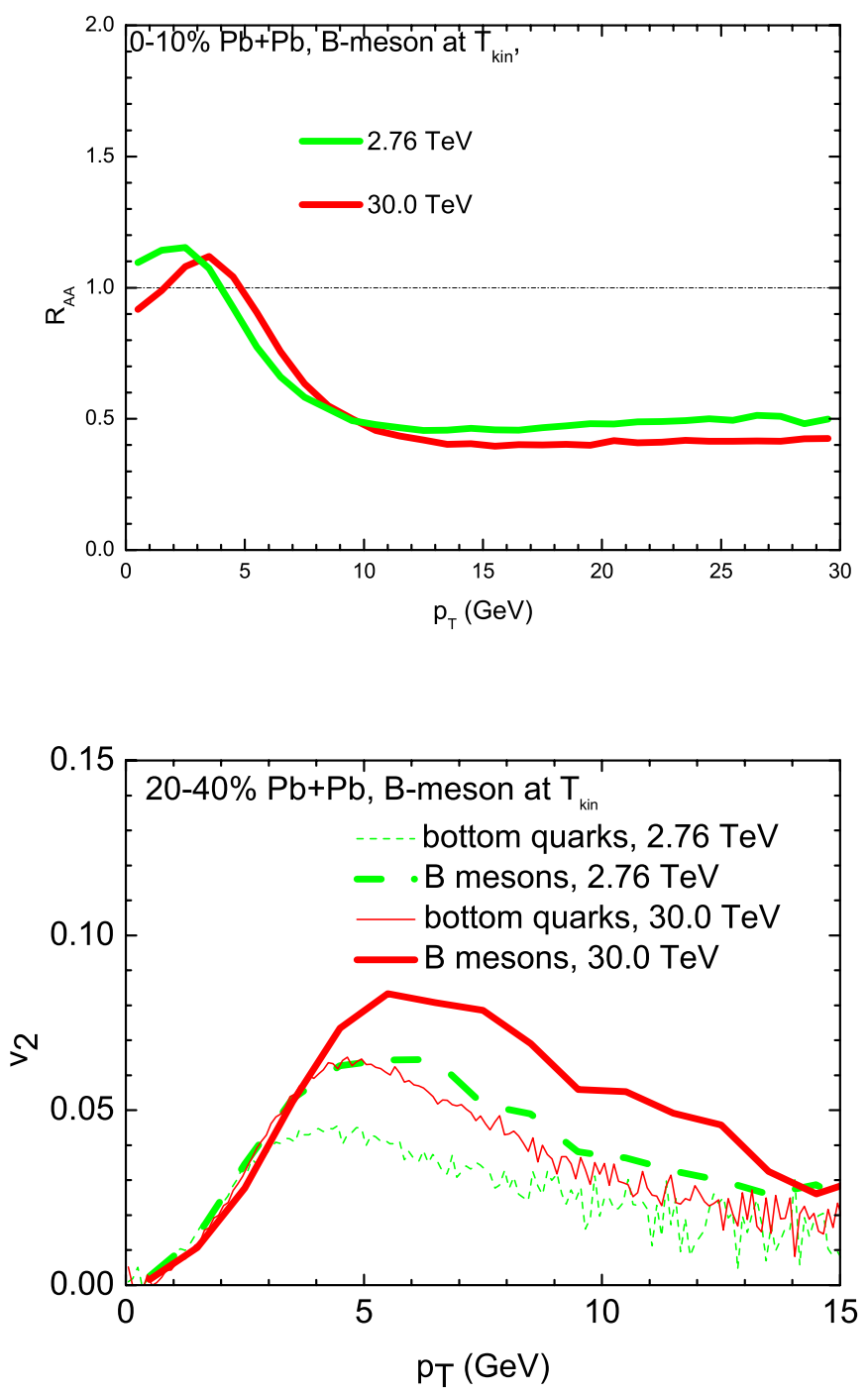

Figure 22: (Color online) The $R_{\mathrm{AA}}$ (upper panel) and $v_{2}$ (lower panel) of bottom quarks and $B$ mesons for semi-central $\mathrm{Pb}+\mathrm{Pb}$ at $\sqrt{s_{N N}}=2.76 \mathrm{TeV}$ and $30 \mathrm{TeV}$.

$\mathrm{TeV}$, one can approximately take thermal charm quark distribution $f_{c}$ in calculating the regeneration rate. Since the regeneration process is the inverse process of the gluon dissociation, the regeneration probability $W_{c \bar{c}}^{g \Psi}$ can be obtained via the detailed balance between the two processes. In the above transport approach, we have neglected elastic collisions between charmonium and the medium, since its effect on the momentum integrated $R_{A A}$ is rather small 201].

The local temperature $T(\mathbf{x}, t)$ and medium velocity $u_{\mu}(\mathbf{x}, t)$ appeared in the thermal gluon and charm quark distributions $f_{g}$ and $f_{c}$ are given by equations of ideal hydrodynamics, $\partial_{\mu} T^{\mu \nu}=0$, where $T_{\mu \nu}$ is the energy-momentum tensor of the medium. While the charm quarks are assumed to be thermalized, they do not reach chemical equilibrium. The space-time evolution of the charm quark density $n_{c}(\mathbf{x}, \tau \mid \mathbf{b})$ satisfies the conservation $\partial_{\mu}\left(n_{c} u^{\mu}\right)=0$, with the initial density determined by the nuclear thickness functions $n_{c}\left(\mathbf{x}, \tau_{0} \mid \mathbf{b}\right)=\left[d \sigma_{N N}^{c(\bar{c})} / d y\right] T_{A}(\mathbf{x}-\mathbf{b} / 2) T_{B}(\mathbf{x}+\mathbf{b} / 2)$.

The shadowing effect becomes extremely important at small $x$ or high colliding energy $\sqrt{s_{N N}}$. In our calculation we use the EKS98 package 202] to take into account of the shadowing factor $R(x)$. Its value in the dominant kinematic region for charm quark production at $\sqrt{s_{N N}}=20 \mathrm{TeV}$ is around 0.8 , which leads to a strong suppression for the regeneration: the charmonium nuclear modification factor is reduced to $\sim 64 \%$ due to the shadowing effect! The other cold nuclear matter effects like Cronin effect [203] and nuclear absorption can also be included in the initial condition of the transport equation [197]. 
The initial thermodynamic conditions for the hydrodynamic evolution is determined by fitting the extrapolated multiplicity of charged hadrons at $\sqrt{s_{N N}}=20 \mathrm{TeV}$. We take the initial thermalization time $\tau_{0}=0.6 \mathrm{fm} / \mathrm{c}$ and the initial temperature at the center of the fireball $T_{0}=540 \mathrm{MeV}$ for central $\mathrm{Pb}+\mathrm{Pb}$ collisions [204]. The critical temperature is chosen as $T_{c}=165 \mathrm{MeV}$.

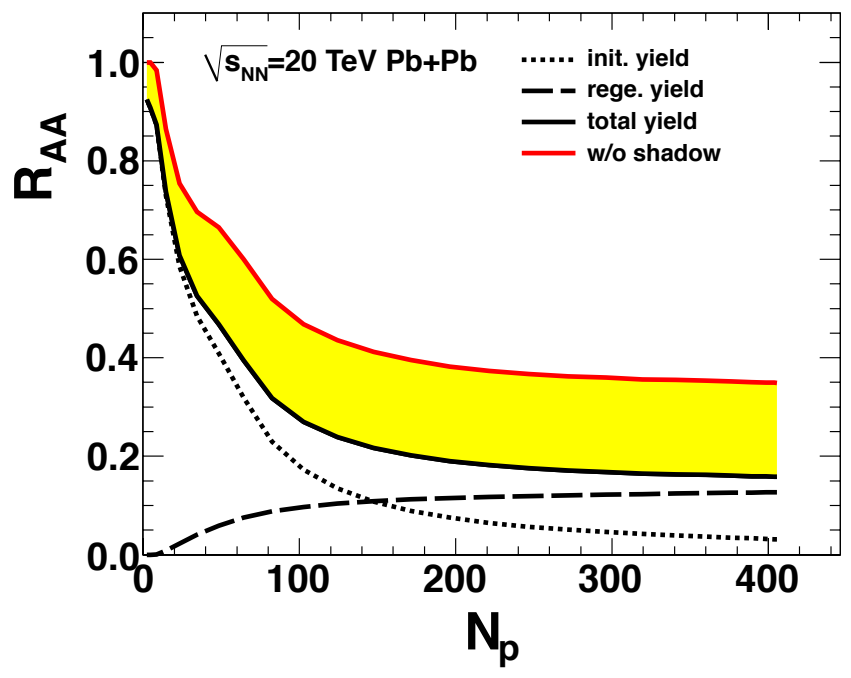

Figure 23: The prompt $J / \psi$ nuclear modification factor as a function of centrality at mid-rapidity $|y|<1$ in $\sqrt{s_{N N}}=20$ TeV $\mathrm{Pb}+\mathrm{Pb}$ collisions. The dotted, dashed and solid lines are the initial production, regeneration and full result, respectively. The upper limit of the band is the full result without shadowing effect.

The initial charmonium distribution is in principle fixed by the corresponding $\mathrm{p}+\mathrm{p}$ data, modified by the cold nuclear matter effects [197]. Since there are not yet $\mathrm{p}+\mathrm{p}$ data at $\sqrt{s_{N N}}=20 \mathrm{TeV}$, we use the simulator PYTHIA [205] to extract the $J / \psi$ and charm quark production cross sections in central rapidity region $|y|<1$,

$$
\frac{d \sigma_{N N}^{J / \psi}}{p_{t} d p_{t}}=A \frac{n-1}{\left\langle\bar{p}_{t}^{2}\right\rangle_{N N}}\left(1+\frac{p_{t}^{2}}{\left\langle\bar{p}_{t}^{2}\right\rangle_{N N}}\right)^{-n}
$$

and $\frac{d \sigma_{N N}^{c \bar{c}}}{d y}=1.4 \mathrm{mb}$, where $\left\langle\bar{p}_{t}^{2}\right\rangle_{N N}=\left\langle p_{t}^{2}\right\rangle_{N N}+a_{g N} l$ is the $J / \psi$ averaged transverse momentum square modified by the Cronin effect with $\left\langle p_{t}^{2}\right\rangle_{N N}=22.69(\mathrm{GeV} / \mathrm{c})^{2}, a_{g N}=0.2 \mathrm{GeV}^{2} / \mathrm{fm}, A=2.011 \times 1.68 \times(10)^{-2} \mathrm{mb}, n=3.164$, and $l$ being the averaged traveling length of the two gluons before they fuse into a $J / \psi$.

The prediction of the nuclear modification factor $R_{A A}$ for $J / \psi$ at $\sqrt{s_{N N}}=20 \mathrm{TeV}$ is shown in Fig. 23 . The initially produced $J / \psi$ 's are almost totally dissolved in central collisions due to the high temperature at mid rapidity. Because of the strong shadowing effect which reduces the charm quark number by a factor of $80 \%$ and the regenerated $J / \psi$ number by a factor of about $64 \%$, the charmonium regeneration is largely suppressed, and the full result is only about $15 \%$ in central collisions. Considering the uncertainty of the calculation of the shadowing effect, the maximum $R_{A A}$ without considering the shadowing effect can reach $35 \%$, see the upper limit of the band in Fig. 23.

The small nuclear modification factor for $J / \psi$ at high energies shown here is caused by the complete melting of initially produced charmonia and strong shadowing effect on initial production of charm quarks and regenerated charmonia. However, the case for $\Upsilon$ may be different. While the maximum temperature $\left(T_{0}=540 \mathrm{MeV}\right)$ of the fireball at $\sqrt{s_{N N}}=20 \mathrm{TeV}$ is several times higher than the $J / \psi$ dissociation temperature $T_{d}^{J / \psi} \sim 1.5 T_{c}$, it is around the $\Upsilon$ dissociation temperature $T_{d}^{\Upsilon} \sim 3 T_{c}$. Therefore, most of the initially produced and regenerated $\Upsilon$ 's can survive the quark matter. The initial number of produced bottom quarks are also smaller leading to smaller number of regenerated $\Upsilon$ 's in the final state. The effect of shadowing on the initial bottom quark production is also expected to be smaller. Therefore, the nuclear modification factor for $\Upsilon$ is expected to be larger than that for $J / \psi$ and increases with collision centrality. 

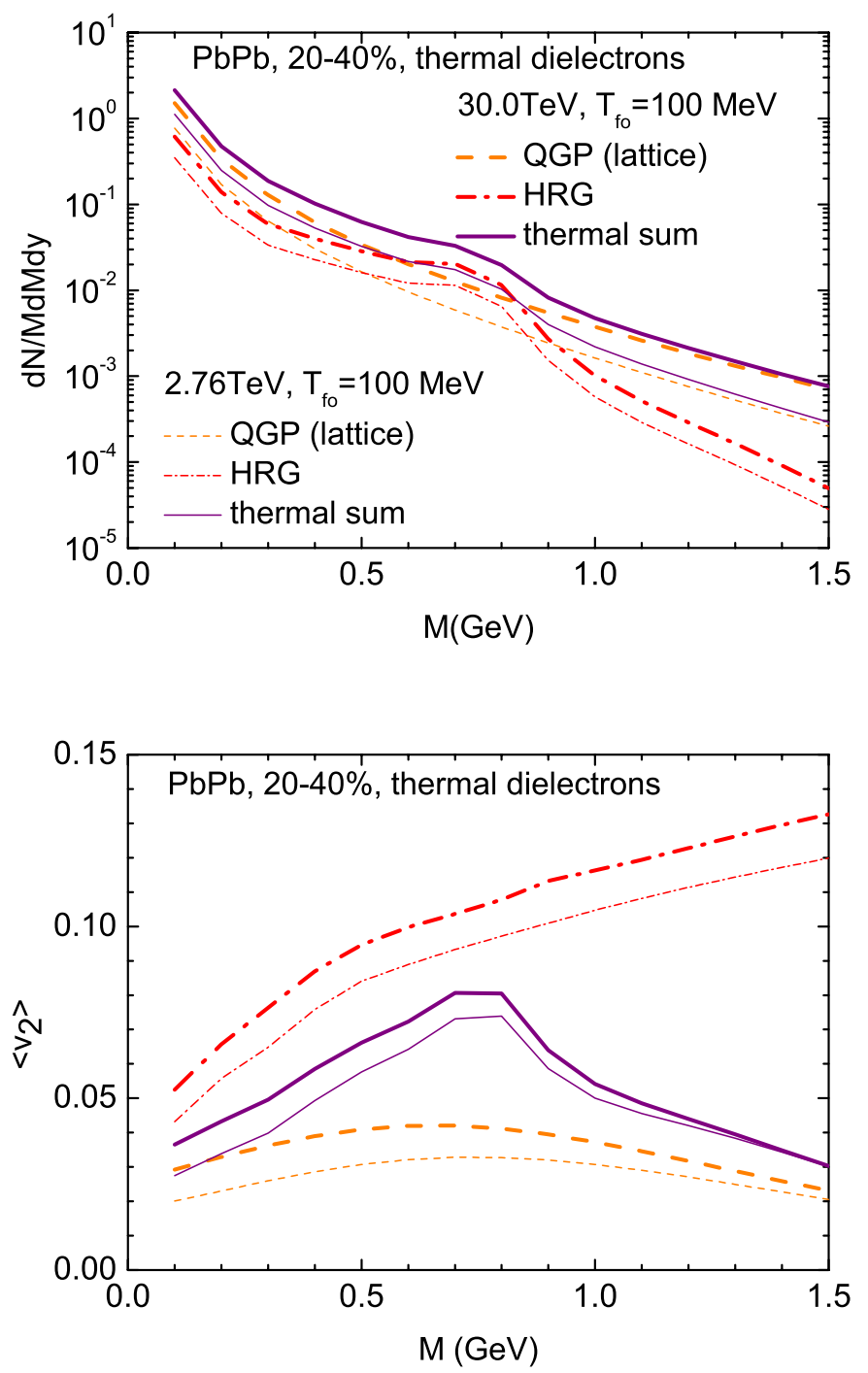

Figure 24: (Color online) The invariant-mass spectra (upper panel) and integrated elliptic flow (lower panel) of thermal dielectrons for semi-central $\mathrm{Pb}+\mathrm{Pb}$ collisions at $\sqrt{s_{\mathrm{NN}}}=2.76 \mathrm{TeV}$ and $30 \mathrm{TeV}$.

\section{ELECTROMAGNETIC EMISSION FROM HEAVY-ION COLLISIONS}

Electromagnetic observables serve as a clean penetrating probe to the ultra-relativistic heavy-ion collisions. Because of the smallness of the electromagnetic coupling compared to the strong interaction, the produced real and virtual photons suffer negligible final-state interactions. Therefore, the radiated thermal photons and dileptons carry direct dynamical information about the early stage of the fireball evolution, which are complementary to the majority of the hadronic observables.

The thermal dilepton emission rate per unit phase space can be written as

$$
\frac{d N_{l^{+} l^{-}}}{d^{4} x d^{4} q}=-\frac{\alpha_{\mathrm{EM}}^{2} L(M)}{\pi^{3} M^{2}} f^{B}\left(q_{0} ; T\right) \operatorname{Im} \Pi_{\mathrm{EM}}\left(M, q ; \mu_{B}, T\right),
$$

where the key quantity is the electromagnatic (EM) spectral function of the QCD medium, $\operatorname{Im} \Pi_{\mathrm{EM}} \equiv \frac{1}{3} g_{\mu \nu} \operatorname{Im} \Pi_{\mathrm{EM}}^{\mu \nu}$, weighted by the thermal Bose factor, $f^{B}$, and the virtual photon propagator, $1 / M^{2}$, with dilepton invariant mass $M^{2}=q_{0}^{2}-q^{2} ; L(M)$ is a lepton phase-space factor (=1 for vanishing lepton mass).

In the low-mass region, and in hadronic matter, the EM spectral function is dominated by the $\rho$ meson, i.e., it is 

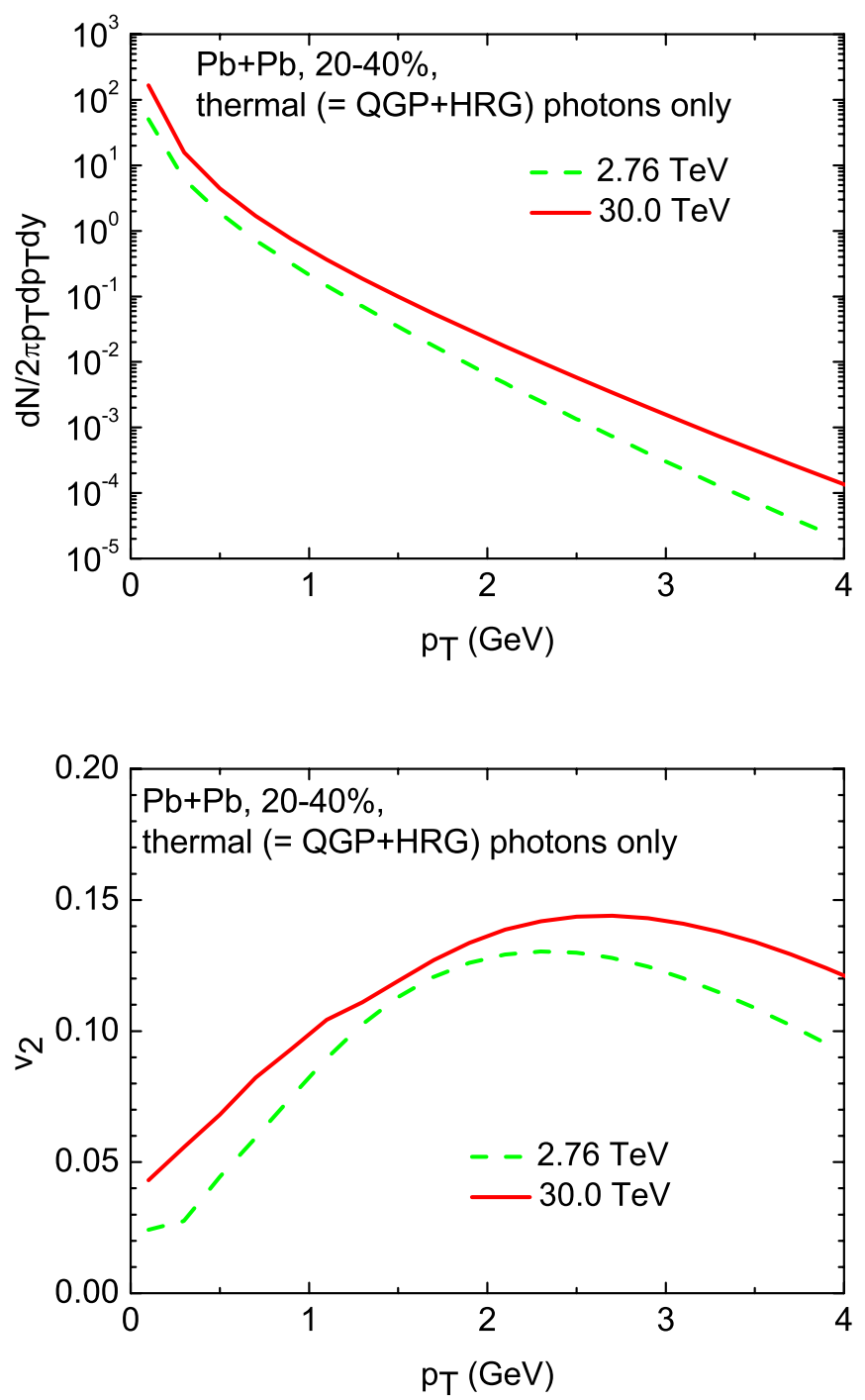

Figure 25: (Color online) The transverse-momentum spectra (upper panel) and elliptic flow (lower panel) of thermal photons for semi-central $\mathrm{Pb}+\mathrm{Pb}$ collisions at $\sqrt{s_{\mathrm{NN}}}=2.76 \mathrm{TeV}$ and $30 \mathrm{TeV}$.

essentially proportional to the imaginary part of the in-medium $\rho$ propagator,

$$
D_{\rho}\left(M, q ; \mu_{B}, T\right)=\frac{1}{M^{2}-m_{\rho}^{2}-\Sigma_{\rho \pi \pi}-\Sigma_{\rho M}-\Sigma_{\rho B}} .
$$

The medium effects are calculated in thermal-field theory through self-energies [206 209] caused by (a) interactions of the $\rho$ 's pion cloud with hadrons from the heat bath $\left(\Sigma_{\rho \pi \pi}\right)$, e.g., $\pi N \rightarrow \Delta$; (b) resonant $\rho$ scattering off thermal mesons $\left(\Sigma_{\rho M}\right)$, e.g., $\rho \pi \rightarrow a_{1}$; and (c) resonant $\rho$ scattering off baryons and anti-baryons $\left(\Sigma_{\rho B}\right)$, e.g., $\rho N \rightarrow N^{*}$. The effective hadronic vertices are constrained by EM gauge invariance and empirical decay branchings and scattering data in vacuum. The off-shell dynamics naturally includes subthreshold excitations, such as $\rho+N \rightarrow N^{*}(1520)$, which are instrumental in populating the low-mass strength in the EM spectral function. The generic outcome of these calculations is a strong broadening of the $\rho$ 's spectral shape, with only small mass shifts (which tend to cancel among the different contributions). For the dilepton emission in QGP, we use the leading-order pQCD rate augmented by a lQCD-inspired form factor [60] (which yields results similar to hard-thermal loop calculations [210]), extended to finite 3-momentum 211. This approach allows for good description of available dielectron emission spectra at SPS and RHIC energies [211, 212].

For thermal photon emission one can find the rates as calculated in Ref. [213]. The hadronic emission was obtained 
by carrying the above-described many-body calculations for dileptons to the photon point, and adding mesonic $t$ channel reactions (which become important at the photon point) from an effective Yang-Mills lagrangian for the $\pi \rho a_{1}$ system, plus $\omega t$-channel exchange in $\pi \rho \rightarrow \pi \gamma$, plus $\pi \pi$ and $\pi K$ Bremsstrahlung [214]. In the vicinity of $T_{\mathrm{pc}}$, these rates approximately match the LO QGP rates [215], thereby rendering a near continuous photon emissivity across the transition region [216], analogous to the dilepton case.

The predicted invariant-mass and transverse-momentum spectra, as well as elliptic flow for thermal EM radiation in $30 \mathrm{TeV} \mathrm{Pb}+\mathrm{Pb}$ collisions are summarized in Figs. 24 and 25 for a medium evolution model according to the TAMUtuned AZHYDRO [187] code as described in Sec.VB. We recall that the early saturation of the energy-momentum anisotropy (cf. discussions in Sec. $\mathrm{VB}$ ) is instrumental for the final $v_{2}$ of the thermal emission, and plays an important role in the understanding of the large direct-photon $v_{2}$ as recently observed by PHENIX [217] and ALICE [218]. We also note that, as discussed in Ref. [216, the continuous hadronic freeze-out in the hydrodynamic evolution may underestimate somewhat the hadronic emission contributions. Nonetheless, compared to the LHC results at $2.76 \mathrm{TeV}$, the thermal low-mass dilepton yield at $30 \mathrm{TeV}$ increases by about a factor of 2 (cf. Fig. 24), which is in line with the stronger than $N_{\mathrm{ch}}$ scaling found in previous calculations [211, and with existing dilepton data at SPS and RHIC. In fact, this behavior allows to utilize the low-mass thermal radiation yield as a unique measure to infer the lifetime of the fireball to within $\sim 10 \%$ [219]. At higher $p_{T}$, e.g., in the thermal photon spectra around $p_{T} \simeq 2 \mathrm{GeV}$ (cf. Fig. 25), the increase in yield becomes even larger due to the increase in radial flow at the higher collision energy.

To take into account of the fluctuation in the initial conditions of the hydrodynamic evolution of the medium on direct photon spectra in $\mathrm{Pb}+\mathrm{Pb}$ collisions at $\sqrt{s_{\mathrm{NN}}}=30 \mathrm{TeV}$ we employ event-by-event iEBE-VISHNU framework [220]. The fluctuating initial entropy density profiles are generated using Monte-Carlo Glauber (MCGlb) and MCKLN models. The spatial configuration of the nucleon positions inside the lead nucleus are sampled with realistic two-body nucleon-nucleon correlations [221]. In MCGlb model, the collision-by-collision multiplicity fluctuation is implemented based on the phenomenological KNO scaling observed in p-p collisions [222. For both initial conditions models, the event centrality is determined by sorting 1 million minimum bias collision events according to their initial total entropy. The generated entropy density are then evolved using $(2+1)$-d viscous hydrodynamic code, VISH2+1 [120], starting at $\tau_{0}=0.6 \mathrm{fm}$. The hydrodynamic equations are numerically solved with a lattice QCD based equation of state (EoS), s95p-v0-PCE 94, which implemented partial chemical equilibrium (PCE) below $T_{\text {chem }}=165 \mathrm{MeV}$. MCGlb initial conditions are evolved with specific shear viscosity, $\eta / s=0.08$ and initial density profiles from MCKLN model are propagated with $\eta / s=0.20$. These two sets of runs gave reasonable description of hadronic flow measurements in $\mathrm{Pb}+\mathrm{Pb}$ collisions at $\sqrt{s_{\mathrm{NN}}}=2.76 \mathrm{TeV}$ [223, 224]. Here, we use them to extrapolate to higher collision energy. The final kinetic freeze-out is chosen to be $T_{\mathrm{dec}}=120 \mathrm{MeV}$. The overall normalization factor is fixed to fit the estimated final charged hadron multiplicity, $d N^{\mathrm{ch}} /\left.d \eta\right|_{|\eta|<0.5}=2700$ at $0-5 \%$ most central centrality.

Thermal photons radiation is then calculated from these calibrated hydrodynamic medium above $T=120 \mathrm{MeV}$. In the QGP phase, the full leading order $O\left(\alpha_{s} \alpha_{\mathrm{EM}}\right)$ photon emission rate is used [215], which includes Compton scattering, quark-anti quark annihilation, and the effective " $1 \rightarrow 2$ " collinear emission. In the hadron gas phase, photon produced through meson-meson reactions in a hadronic $\left(\pi, K, \rho, \omega, K^{*}, a_{1}\right)$ gas [213], through the medium broadened $\rho$-spectral function, and through $\pi+\pi$ bremsstrahlung [214, 225] are taken into account. Because the hydrodynamic medium is assumed to be slightly out-of-equilibrium, shear viscous corrections to the photon production rates are included in the 2 to 2 scattering processes in the QGP phase [226] and in all the mesonic reaction channels in the hadronic phase [227]. The viscous corrections to the other channels have not been derived in theory yet. We use the QGP photon emission rate for the temperature region above $180 \mathrm{MeV}$ and switch to hadron gas rate below. In each collision event, the thermal photon spectrum is calculated by convoluting the photon emission rates with the hydrodynamic medium,

$$
E \frac{d N^{\mathrm{th}, \gamma}}{d^{3} p}=\left.\int d^{4} x\left(q \frac{d R}{d^{3} q}(q, T(x))\right)\right|_{q=p \cdot u(x)}
$$

The anisotropy flow coefficients of the thermal photon momentum distribution are computed using the scalar-product method, $v_{n}\{\mathrm{SP}\}$. We correlate every produced thermal photon with the reference flow vector constructed using all charged hadrons [228,

$$
v_{n}\{\operatorname{SP}\}\left(p_{T}\right)=\frac{\left\langle\frac{d N^{\gamma}}{d y p_{T} d p_{T}} v_{n}^{\gamma}\left(p_{T}\right) v_{n}^{\mathrm{ch}} \cos \left(n\left(\Psi_{n}^{\gamma}\left(p_{T}\right)-\Psi_{n}^{\mathrm{ch}}\right)\right)\right\rangle}{\left\langle\frac{d N^{\gamma}}{d y p_{T} d p_{T}}\right\rangle v_{n}^{\mathrm{ch}}\{2\}}
$$

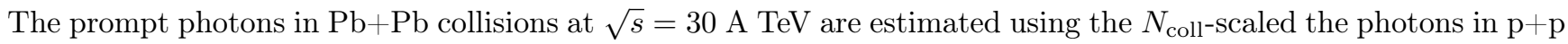
collisions at the same collision energy. The direct photon production in $\mathrm{p}+\mathrm{p}$ collisions are calculated using the Nextto-leading-order (NLO) pQCD. The factorization scales in the parton distribution function, $\mu_{f}$, and fragmentation function, $\mu_{D}$, are chosen at $2 \mathrm{GeV}$, which also sets the lower limit for the calculable $p_{T}$, via the employed scale 
variations, $\mu=2 p_{T}$. The nuclear effects, such as shadowing and isospin effects, in the parton distribution function are not included in the current estimation because their effects are genuinely small and the uncertainty becomes large at such a high collision energy.
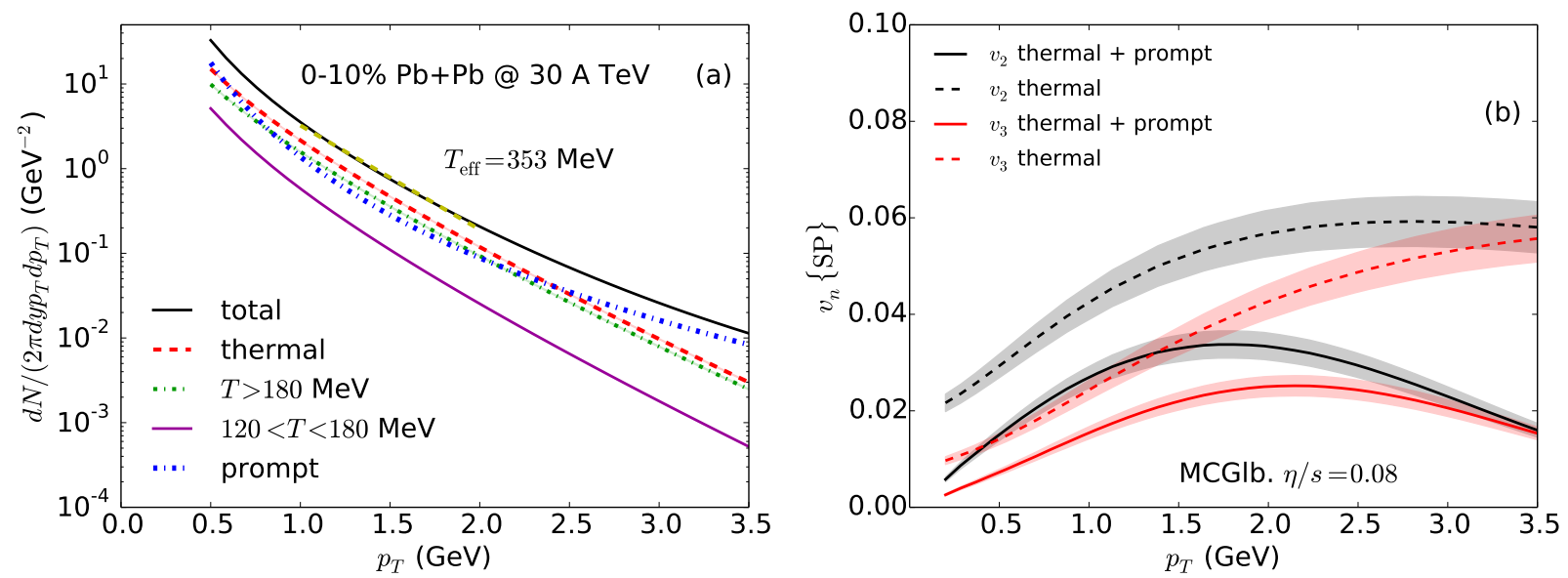

Figure 26: Direct photon spectra and anisotropic flow coefficients $v_{2,3}\{\mathrm{SP}\}$ at $0-10 \%$ centrality $\mathrm{Pb}+\mathrm{Pb}$ collisions at $\sqrt{s_{\mathrm{NN}}}=30$ $\mathrm{TeV}$ using MCGlb model with $\eta / s=0.08$. Individual contributions of thermal photons are shown. The prompt photons are

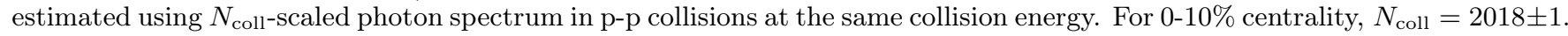

Fig. 26a shows the the direct photon spectrum in $0-10 \%$ centrality $\mathrm{Pb}+\mathrm{Pb}$ collisions at $\sqrt{s_{\mathrm{NN}}}=30 \mathrm{TeV}$. We find that the thermal signal exceed the prompt photon contribution for $p_{T}<2.5 \mathrm{GeV}$. Most the of the thermal photons come from the high temperature region $T>180 \mathrm{MeV}$. The hadronic phase, $120<T<180 \mathrm{MeV}$ contributes about $10 \%$ to the total thermal photons. Because of the strong hydrodynamic radial flow and high peak temperature of the fireball, the inverse slope of the direct photon spectrum reaches $353 \mathrm{MeV}$, which is $\sim 50 \mathrm{MeV}$ higher than the inverse slope of direct photon spectrum at $2.76 \mathrm{~A} \mathrm{TeV}$ [229, 230]. In Fig. 26p, we show the direct photon anisotropic flow coefficients, $v_{2,3}\{\mathrm{SP}\}\left(p_{T}\right)$. Thermal components are shown for comparison. The thermal photon anisotropic flows are smaller than hadronic ones as shown in IIIB. This is because the most of thermal photons are emitted from early $T>180 \mathrm{MeV}$ region, where the hydrodynamic flow has not fully developed yet. Thus they carry less flow anisotropy compared with the hadrons. The triangular flow of direct photon are driven by the event-by-event fluctuation. Its signal is comparable with elliptic flow in the $0-10 \%$ central collisions. Comparing the $v_{n}$ of thermal and direct photons, we find the prompt photons dilute $\sim 50 \%$ of the flow anisotropy in the final direct photon signals.

Fig. 27] shows our calculations of the direct photons emitted in $0-40 \%$ centrality bin for two different sets of initial
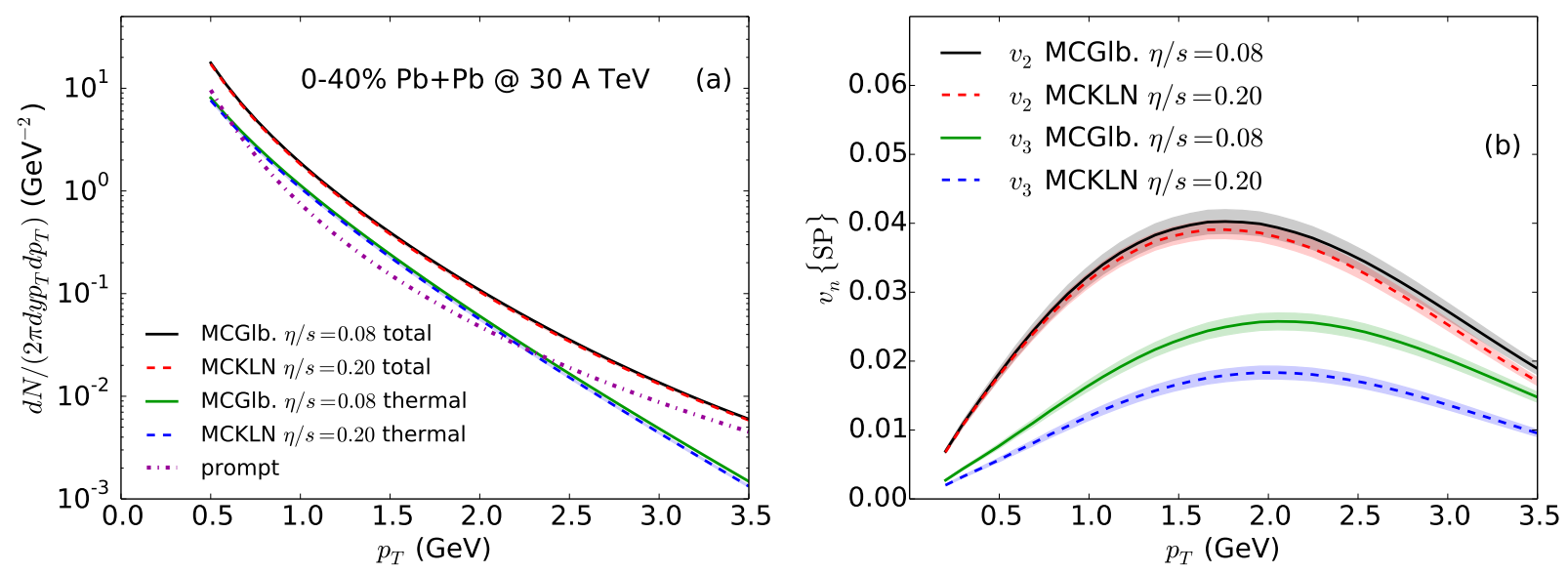

Figure 27: Prediction of direct photon spectra and anisotropic flow coefficients $v_{2,3}\{\mathrm{SP}\}\left(p_{T}\right)$ at $0-40 \%$ centrality $\mathrm{Pb}+\mathrm{Pb}$ collisions at $\sqrt{s_{\mathrm{NN}}}=30 \mathrm{TeV}$ from MCGlb model with $\eta / s=0.08$ and MCKLN model with $\eta / s=0.20$. The prompt

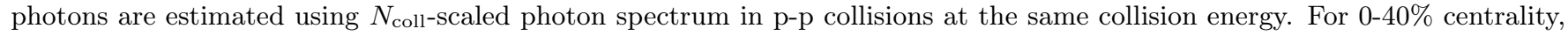
$N_{\text {coll }}=1092 \pm 1$ 
conditions. The two initial conditions with their corresponding specific shear viscosity give very close predictions for the direct photon spectrum and elliptic flow coefficient, $v_{2}\{\mathrm{SP}\}\left(p_{T}\right)$. The MCKLN initial conditions with a larger $\eta / s$ produce a smaller direct photon triangular flow.
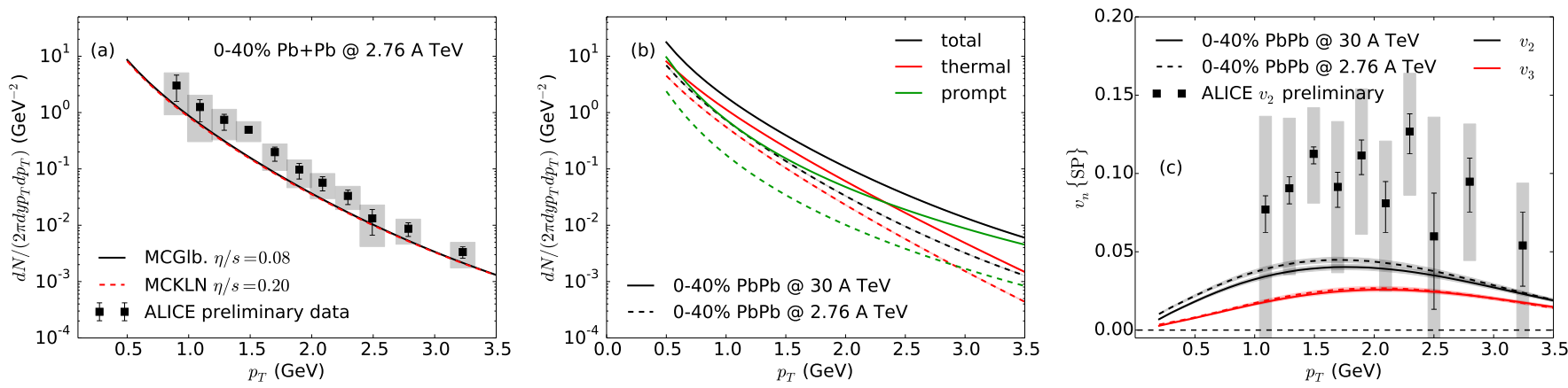

Figure 28: Panel (a): Theory calculations of direct photon spectra compared with ALICE preliminary measurement in 0-40\% $\mathrm{Pb}+\mathrm{Pb}$ collisions at 2.76 A TeV [229. Panel (b): Comparisons of the individual component in direct photon spectra in $0-40 \%$ $\mathrm{Pb}+\mathrm{Pb}$ collisions at $30 \mathrm{~A} \mathrm{TeV}$ and at $2.76 \mathrm{~A} \mathrm{TeV}$. Panel (c): Direct photon $v_{2,3}\{\mathrm{SP}\}\left(p_{T}\right)$ in $0-40 \% \mathrm{~Pb}+\mathrm{Pb}$ collisions at $30 \mathrm{~A}$ $\mathrm{TeV}$ and at $2.76 \mathrm{~A} \mathrm{TeV}$. Direct photon $v_{2}\{\mathrm{SP}\}\left(p_{T}\right)$ at $2.76 \mathrm{~A} \mathrm{TeV}$ is compared with ALICE preliminary measurement 218 .

In Fig. 28, we compare the direct photon spectra and their anisotropic flow coefficients in $0-40 \% \mathrm{~Pb}+\mathrm{Pb}$ collisions at $\sqrt{s}=30 \mathrm{ATeV}$ with those at $\sqrt{s}=2.76 \mathrm{ATeV}$ available at current LHC experiments. Because of $\sim 70 \%$ more entropy in the system, the space-time volume of the hydrodynamic medium is considerably larger compared with the fireball at $\sqrt{s}=2.76 \mathrm{ATeV}$. The lifetime of the fireball is $\sim 30 \%$ longer. Therefore, there are about 2.5 times thermal photons produced compared to current LHC energy. However, we find a even large increase of the prompt photons, about a factor of 4 , compared to $\sqrt{s}=2.76 \mathrm{ATeV}$. The ratio of thermal/prompt reduces as the collision energy increases. Because of this large prompt component, the direct photon anisotropic flow coefficients are slightly smaller compared to current LHC energy. The final produced direct photon spectrum at $\sqrt{s}=30 \mathrm{ATeV}$ is roughly 3.5 times of the photon produced at $\sqrt{s}=2.76 \mathrm{ATeV}$.
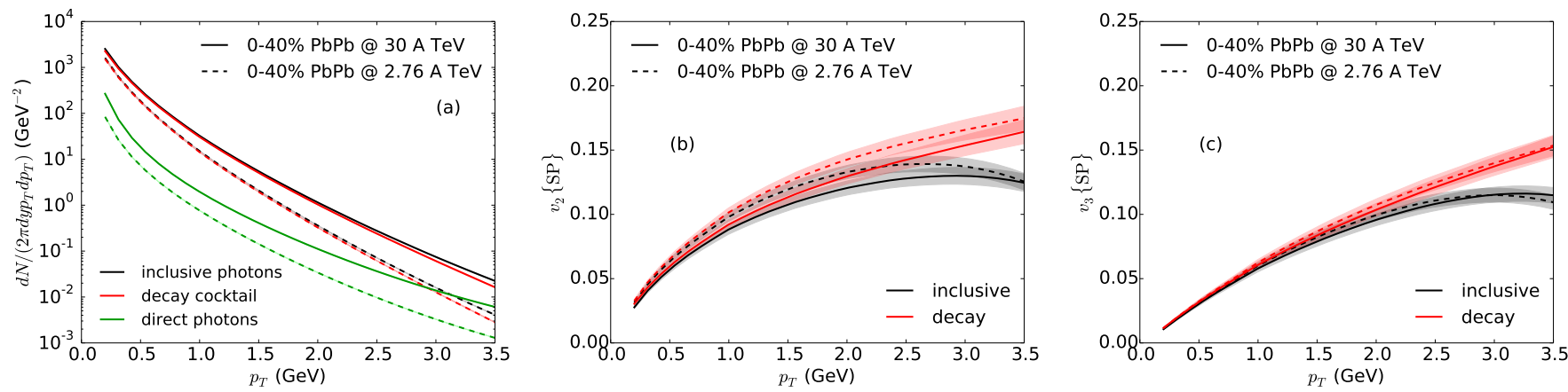

Figure 29: Comparisons of the inclusive, decay, and direct photon spectra and $v_{n}\{\mathrm{SP}\}\left(p_{T}\right)$ between $0-40 \% \mathrm{~Pb}+\mathrm{Pb}$ collisions at $30 \mathrm{~A} \mathrm{TeV}$ and at $2.76 \mathrm{~A} \mathrm{TeV}$.

In Figs. 29, we compare the inclusive photon spectra as well as the decay cocktail between $\mathrm{Pb}+\mathrm{Pb}$ collisions at 30 $\mathrm{A} \mathrm{TeV}$ and at $2.76 \mathrm{~A} \mathrm{TeV}$. In Fig. $29 \mathrm{k}$, we find that the increase of direct photon production is larger compared to the increase in the inclusive photons. The signal to background ratio for direct photons increases as collision energy increases. This makes the direct photon measurement easier at $30 \mathrm{~A} \mathrm{TeV}$. In Figs. 29p,c, we make predictions for the inclusive and decay photon $v_{2,3}\{\mathrm{SP}\}\left(p_{T}\right)$. Similar to hadrons, the $p_{T}$-differential anisotropic flows of inclusive and decay photons are very close the ones in $\mathrm{Pb}+\mathrm{Pb}$ collisions at $2.76 \mathrm{~A} \mathrm{TeV}$.

\section{SUMMARY}

Since the discovery of the strongly coupled QGP at RHIC about a decode ago, experimental and theoretical efforts in high-energy nuclear physics have been focused on the quantitative study of the properties of the sQGP at 
extremely high temperatures. These include the extraction of the shear viscosity to entropy density ratio of the bulk QGP medium, the jet transport parameter for energetic jets propagating inside the QGP medium, and the diffusion coefficient for heavy-flavor particles in QCD matter as formed in high-energy heavy-ion collisions at both RHIC and LHC. The future frontier of heavy-ion collisions will be at both lower and very-high colliding energy regimes. For the latter, such as that in the beam energy scan (BES) program at RHIC and at FAIR, one expects to reach the highest baryon density in heavy-ion collisions, to explore the phase structure of QCD matter, in particular search for signals of a critical end-point in the QCD phase transition. At the high-energy frontier, one expects to increase the initial temperatures that are currently possible at RHIC and LHC in the central region of the two colliding nuclei. Under these conditions, the properties of QGP medium might approach that of weakly interacting quarks and gluons. According to predictions by pQCD, the ratio of shear viscosity to entropy density and the heavy-flavor diffusion coefficient (scaled by $1 / T$ ) should increase, while the jet transport parameter (scaled by $T^{3}$ ) should decrease.

Using HIJING and CGC models, we have estimated the final charged-hadron multiplicity in central $\mathrm{Pb}+\mathrm{Pb}$ collisions at $\sqrt{s}=30 \mathrm{TeV}$ to be about $70 \%$ larger than at the current LHC energy $(\sqrt{s}=2.76 \mathrm{TeV})$. Assuming the initial thermalization time to be the same as at the LHC, $\tau_{0}=0.6 \mathrm{fm} / c$, the initial temperature of a thermalized QGP at $\sqrt{s}=30 \mathrm{TeV}$ will be about $T_{0} \approx 560 \mathrm{MeV}$. Based on our calculations of the anisotropic flow of charged hadrons using an event-by-event $3+1 \mathrm{D}$ ideal hydrodynamic model with fluctuating initial conditions, we expect to see strong signals of higher harmonic flow which should provide stringent constraints on the shear viscosity. We have also calculated the suppression factors for charged hadrons with large transverse momentum in central $\mathrm{Pb}+\mathrm{Pb}$ collisions at $\sqrt{s}=30$ $\mathrm{TeV}$ within two different approaches to energy loss. The suppression factor is found to continue to decrease over a large range of transverse momenta, and thus provides sufficient sensitivity to determine the jet transport parameter at such high initial temperatures. Though the suppression of full jet production is not as sensitive to the increase in initial temperature, the jet profile function is found to be significantly modified and should provide additional constraints on properties of the QGP medium. For open heavy flavor, both the high- $p_{T}$ suppression and the elliptic flow are expected to increase by about $20 \%$. The final $J / \psi$ yield in $\mathrm{Pb}+\mathrm{Pb}$ collisions at $\sqrt{s}=30 \mathrm{TeV}$ is predicted to be more strongly dominated by regeneration from the recombination of initially produced charm quarks, due to a near-complete suppression of the initially produced $J / \psi$. The final $J / \psi$ nuclear modification factor, however, might turn out to be smaller than that at LHC due to an expected suppression of initial charm quark production by the gluon nuclear shadowing. It will therefore be essential to determine gluon shadowing from $\mathrm{p}+\mathrm{A}$ and $\mathrm{e}+\mathrm{A}$ collisions in order to reliably quantify the mechansims for $J / \psi$ 's regeneration in the QGP medium. The calculations of electromagnetic radiation from the medium show a more pronounced increase in yields as the final spectra receive significant contributions throughout the entire fireball evolution. For example, low-mass dilepton yields increase by a factor of $\sim 2$ and allow for a "measurement" of the increased fireball lifetime at higher colliding energies.

To conclude, based on the calculations presented here, a systematic study of the above experimental observables at a future very high energy heavy-ion collider will provide us with an opportunity to significantly improve our understanding of the properties of the QGP and, in particular, open a window on the weakly interacting limit of QGP at very high temperature.

\section{Acknowledgements}

Work in this paper is partially supported by the NSFC under Grant No. 11175071, No. 11221504, No. 11305089, No. 11322546, No. 11375072, No. 11435001, No. 11435004, China MOST under Grant No. 2014DFG02050, No. 2015CB856900, the Major State Basic Research Development Program in China (No. 2014 CB845404 and No. 2014CB845403), the Natural Sciences and Engineering Research Council of Canada, the US National Science Foundation under grant number PHY-1306359, the Director, Office of Energy Research, Office of High Energy and Nuclear Physics, Division of Nuclear Physics, of the U.S. Department of Energy under Contract Nos. DE-AC0205CH11231, de-sc0012704 and within the framework of the JET Collaboration. BJS is also supported by a DOE Office of Science Early Career Award.

[1] H.-T. Ding, F. Karsch, and S. Mukherjee, (2015), arXiv:arXiv:1504.05274.

[2] PHENIX Collaboration, K. Adcox et al., Nucl.Phys. A757, 184 (2005), arXiv:nucl-ex/0410003

[3] STAR Collaboration, J. Adams et al., Nucl.Phys. A757, 102 (2005), arXiv:nucl-ex/0501009.

[4] P. Jacobs and X.-N. Wang, Prog.Part.Nucl.Phys. 54, 443 (2005), arXiv:hep-ph/0405125.

[5] U. Heinz and R. Snellings, Ann.Rev.Nucl.Part.Sci. 63, 123 (2013), arXiv:1301.2826.

[6] X.-N. Wang, Nucl.Phys. A750, 98 (2005), arXiv:nucl-th/0405017.

[7] PHEnIX, A. Adare et al., Phys. Rev. Lett. 98, 172301 (2007), arXiv:nucl-ex/0611018 
[8] STAR Collaboration, B. Abelev, Science 328, 58 (2010), arXiv:1003.2030

[9] N. Armesto et al., J.Phys. G35, 054001 (2008), arXiv:0711.0974.

[10] B. Muller, J. Schukraft, and B. Wyslouch, Ann.Rev.Nucl.Part.Sci. 62, 361 (2012), arXiv:1202.3233.

[11] H. Song, S. A. Bass, and U. Heinz, Phys.Rev. C83, 054912 (2011), arXiv:1103.2380.

[12] C. Gale, S. Jeon, B. Schenke, P. Tribedy, and R. Venugopalan, Phys.Rev.Lett. 110, 012302 (2013), arXiv:1209.6330.

[13] JET, K. M. Burke et al., Phys.Rev. C90, 014909 (2014), arXiv:1312.5003

[14] See http://vlhc.org (2015), arXiv:.

[15] See http://cepc.ihep.ac.cn (2015), arXiv:.

[16] T. Bhattacharya et al., Phys.Rev.Lett. 113, 082001 (2014), arXiv:1402.5175

[17] G. Cossu et al., Phys.Rev. D87, 114514 (2013), arXiv:1304.6145.

[18] HotQCD Collaboration, A. Bazavov et al., Phys.Rev. D86, 094503 (2012), arXiv:1205.3535

[19] M. I. Buchoff et al., Phys.Rev. D89, 054514 (2014), arXiv:1309.4149.

[20] J. Engels, F. Karsch, H. Satz, and I. Montvay, Phys.Lett. B101, 89 (1981).

[21] HotQCD Collaboration, A. Bazavov et al., Phys.Rev. D90, 094503 (2014), arXiv:1407.6387

[22] S. Borsanyi et al., Phys.Lett. B730, 99 (2014), arXiv:1309.5258

[23] A. Bazavov et al., Phys.Rev. D85, 054503 (2012), arXiv:1111.1710.

[24] Wuppertal-Budapest Collaboration, S. Borsanyi et al., JHEP 1009, 073 (2010), arXiv:1005.3508

[25] A. Bazavov et al., Phys. Rev. D88, 094021 (2013), arXiv:1309.2317.

[26] H. T. Ding, S. Mukherjee, H. Ohno, P. Petreczky, and H. P. Schadler, (2015), arXiv:1507.06637.

[27] R. Bellwied et al., (2015), arXiv:1507.04627.

[28] O. Philipsen, Prog. Part. Nucl. Phys. 70, 55 (2013), arXiv:1207.5999

[29] S. Borsányi et al., PoS LATTICE2014, 224 (2015), arXiv:1410.7917.

[30] S. Ejiri et al., Phys.Rev. D80, 094505 (2009), arXiv:0909.5122.

[31] H.-T. Ding et al., PoS LATTICE2013, 157 (2014), arXiv:1312.0119

[32] R. D. Pisarski and F. Wilczek, Phys.Rev. D29, 338 (1984).

[33] S. Aoki, H. Fukaya, and Y. Taniguchi, Phys.Rev. D86, 114512 (2012), arXiv:1209.2061

[34] H. Ding et al., Phys.Rev. D86, 014509 (2012), arXiv:1204.4945

[35] A. Bazavov, F. Karsch, Y. Maezawa, S. Mukherjee, and P. Petreczky, (2014), arXiv:1411.3018.

[36] S. Borsanyi et al., JHEP 1404, 132 (2014), arXiv:1401.5940.

[37] G. Aarts et al., Phys.Rev.Lett. 106, 061602 (2011), arXiv:1010.3725.

[38] G. Aarts et al., JHEP 1312, 064 (2013), arXiv:1310.5467.

[39] G. Aarts et al., JHEP 1303, 084 (2013), arXiv:1210.2903.

[40] G. Aarts et al., JHEP 1407, 097 (2014), arXiv:1402.6210.

[41] G. Aarts et al., JHEP 1111, 103 (2011), arXiv:1109.4496.

[42] S. Kim, P. Petreczky, and A. Rothkopf, (2014), arXiv:1409.3630

[43] M. Laine, O. Philipsen, P. Romatschke, and M. Tassler, JHEP 0703, 054 (2007), arXiv:hep-ph/0611300

[44] A. Beraudo, J.-P. Blaizot, and C. Ratti, Nucl.Phys. A806, 312 (2008), arXiv:0712.4394

[45] N. Brambilla, J. Ghiglieri, A. Vairo, and P. Petreczky, Phys.Rev. D78, 014017 (2008), arXiv:0804.0993.

[46] Y. Burnier, O. Kaczmarek, and A. Rothkopf, (2014), arXiv:1410.2546

[47] Y. Burnier and A. Rothkopf, Phys.Rev.Lett. 111, 182003 (2013), arXiv:1307.6106

[48] A. Rothkopf, T. Hatsuda, and S. Sasaki, Phys.Rev.Lett. 108, 162001 (2012), arXiv:1108.1579

[49] Y. Burnier and A. Rothkopf, Phys.Rev. D86, 051503 (2012), arXiv:1208.1899

[50] A. Bazavov et al., Phys.Rev.Lett. 111, 082301 (2013), arXiv:1304.7220

[51] A. Bazavov et al., Phys.Lett. B737, 210 (2014), arXiv:1404.4043.

[52] R. Bellwied, S. Borsanyi, Z. Fodor, S. D. Katz, and C. Ratti, Phys.Rev.Lett. 111, 202302 (2013), arXiv:1305.6297

[53] A. Bazavov et al., Phys.Rev.Lett. 109, 192302 (2012), arXiv:1208.1220

[54] S. Borsanyi et al., Phys.Rev.Lett. 113, 052301 (2014), arXiv:1403.4576.

[55] A. Bazavov et al., Phys.Rev.Lett. 113, 072001 (2014), arXiv:1404.6511.

[56] H. Ding et al., J.Phys. G38, 124070 (2011), arXiv:1107.0311.

[57] H.-T. Ding, EPJ Web Conf. 36, 00008 (2012), arXiv:1207.5187.

[58] H.-T. Ding, (2014), arXiv:1404.5134.

[59] M. Asakawa, T. Hatsuda, and Y. Nakahara, Prog.Part.Nucl.Phys. 46, 459 (2001), arXiv:hep-lat/0011040.

[60] H.-T. Ding et al., Phys.Rev. D83, 034504 (2011), arXiv:1012.4963.

[61] H.-T. Ding, O. Kaczmarek, and F. Meyer, (2014), arXiv:1412.5869.

[62] B. B. Brandt, A. Francis, H. B. Meyer, and H. Wittig, JHEP 1303, 100 (2013), arXiv:1212.4200

[63] G. Aarts et al., (2014), arXiv:1412.6411

[64] A. Amato et al., Phys.Rev.Lett. 111, 172001 (2013), arXiv:1307.6763.

[65] S. Caron-Huot, M. Laine, and G. D. Moore, JHEP 0904, 053 (2009), arXiv:0901.1195.

[66] A. Francis, O. Kaczmarek, M. Laine, and J. Langelage, PoS LATTICE2011, 202 (2011), arXiv:1109.3941.

[67] D. Banerjee, S. Datta, R. Gavai, and P. Majumdar, Phys.Rev. D85, 014510 (2012), arXiv:1109.5738

[68] O. Kaczmarek, (2014), arXiv:1409.3724.

[69] H. B. Meyer, Phys.Rev. D76, 101701 (2007), arXiv:0704.1801.

[70] H. B. Meyer, Phys.Rev.Lett. 100, 162001 (2008), arXiv:0710.3717.

[71] H. B. Meyer, JHEP 0401, 030 (2004), arXiv:hep-lat/0312034. 
[72] G. D. Moore and K. A. Sohrabi, Phys.Rev.Lett. 106, 122302 (2011), arXiv:1007.5333

[73] G. D. Moore and K. A. Sohrabi, JHEP 1211, 148 (2012), arXiv:1210.3340.

[74] G. Denicol, H. Niemi, E. Molnar, and D. Rischke, Phys.Rev. D85, 114047 (2012), arXiv:1202.4551.

[75] CDF Collaboration, F. Abe et al., Phys.Rev. D41, 2330 (1990).

[76] PHOBOS Collaboration, R. Nouicer et al., J.Phys. G30, S1133 (2004), arXiv:nucl-ex/0403033.

[77] ALICE Collaboration, K. Aamodt et al., Eur.Phys.J. C65, 111 (2010), arXiv:0911.5430.

[78] W.-T. Deng, X.-N. Wang, and R. Xu, Phys.Rev. C83, 014915 (2011), arXiv:1008.1841.

[79] X.-N. Wang and M. Gyulassy, Phys.Rev. D44, 3501 (1991).

[80] M. Gyulassy and X.-N. Wang, Comput.Phys.Commun. 83, 307 (1994), arXiv:nucl-th/9502021.

[81] P. F. Kolb, J. Sollfrank, and U. W. Heinz, Phys.Rev. C62, 054909 (2000), arXiv:hep-ph/0006129.

[82] L. D. McLerran and R. Venugopalan, Phys.Rev. D49, 2233 (1994), arXiv:hep-ph/9309289.

[83] D. Kharzeev and M. Nardi, Phys.Lett. B507, 121 (2001), arXiv:nucl-th/0012025.

[84] D. Kharzeev and E. Levin, Phys.Lett. B523, 79 (2001), arXiv:nucl-th/0108006.

[85] H.-J. Drescher and Y. Nara, Phys.Rev. C75, 034905 (2007), arXiv:nucl-th/0611017.

[86] I. Balitsky, Nucl.Phys. B463, 99 (1996), arXiv:hep-ph/9509348

[87] Y. V. Kovchegov, Phys.Rev. D60, 034008 (1999), arXiv:hep-ph/9901281.

[88] J. L. ALbacete and A. Dumitru, (2010), arXiv:1011.5161.

[89] J. L. Albacete, A. Dumitru, H. Fujii, and Y. Nara, Nucl.Phys. A897, 1 (2013), arXiv:1209.2001.

[90] B. Schenke, P. Tribedy, and R. Venugopalan, Phys.Rev.Lett. 108, 252301 (2012), arXiv:1202.6646

[91] B. Schenke, P. Tribedy, and R. Venugopalan, Phys.Rev. C86, 034908 (2012), arXiv:1206.6805.

[92] B. Schenke, P. Tribedy, and R. Venugopalan, Phys.Rev. C89, 024901 (2014), arXiv:1311.3636.

[93] F. Gelis, Nucl.Phys. A931, 73 (2014), arXiv:1412.0471

[94] P. Huovinen and P. Petreczky, Nucl.Phys. A837, 26 (2010), arXiv:0912.2541

[95] J.-Y. Ollitrault, Phys.Rev. D46, 229 (1992).

[96] P. Romatschke and U. Romatschke, Phys.Rev.Lett. 99, 172301 (2007), arXiv:0706.1522

[97] H. Song, S. A. Bass, U. Heinz, T. Hirano, and C. Shen, Phys.Rev.Lett. 106, 192301 (2011), arXiv:1011.2783

[98] G. Policastro, D. T. Son, and A. O. Starinets, Phys.Rev.Lett. 87, 081601 (2001), arXiv:hep-th/0104066.

[99] PHENIX Collaboration, A. Adare et al., Phys.Rev.Lett. 107, 252301 (2011), arXiv:1105.3928

[100] STAR Collaboration, Y. Pandit, Nucl.Phys. A904-905, 357c (2013), arXiv:1210.5315

[101] ATLAS Collaboration, G. Aad et al., Phys.Rev. C86, 014907 (2012), arXiv:1203.3087.

[102] L. Pang, Q. Wang, and X.-N. Wang, Phys.Rev. C86, 024911 (2012), arXiv:1205.5019

[103] B. Zhang, C. Ko, B.-A. Li, and Z.-w. Lin, Phys.Rev. C61, 067901 (2000), arXiv:nucl-th/9907017.

[104] L.-G. Pang, G.-Y. Qin, V. Roy, X.-N. Wang, and G.-L. Ma, Phys. Rev. C91, 044904 (2015), arXiv:1410.8690.

[105] C. Gale, S. Jeon, and B. Schenke, Int.J.Mod.Phys. A28, 1340011 (2013), arXiv:1301.5893.

[106] H. Kowalski, T. Lappi, and R. Venugopalan, Phys.Rev.Lett. 100, 022303 (2008), arXiv:0705.3047

[107] B. Schenke, S. Jeon, and C. Gale, Phys.Rev. C82, 014903 (2010), arXiv:1004.1408.

[108] B. Schenke, S. Jeon, and C. Gale, Phys.Rev.Lett. 106, 042301 (2011), arXiv:1009.3244

[109] B. Schenke, S. Jeon, and C. Gale, Phys.Rev. C85, 024901 (2012), arXiv:1109.6289.

[110] X. Zhu, F. Meng, H. Song, and Y.-X. Liu, Phys.Rev. C91, 034904 (2015), arXiv:1501.03286

[111] ALICE, B. Abelev et al., Phys.Rev. C88, 044910 (2013), arXiv:1303.0737

[112] ALICE, B. B. Abelev et al., Phys.Rev.Lett. 111, 222301 (2013), arXiv:1307.5530.

[113] ALICE, B. B. Abelev et al., Phys.Lett. B728, 216 (2014), arXiv:1307.5543.

[114] P. Braun-Munzinger, D. Magestro, K. Redlich, and J. Stachel, Phys.Lett. B518, 41 (2001), arXiv:hep-ph/0105229.

[115] A. Andronic, P. Braun-Munzinger, and J. Stachel, Nucl.Phys. A772, 167 (2006), arXiv:nucl-th/0511071.

[116] J. Letessier and J. Rafelski, Eur.Phys.J. A35, 221 (2008), arXiv:nucl-th/0504028

[117] H. Song, S. Bass, and U. W. Heinz, Phys.Rev. C89, 034919 (2014), arXiv:1311.0157.

[118] H. Song, S. A. Bass, and U. Heinz, Phys.Rev. C83, 024912 (2011), arXiv:1012.0555.

[119] H. Song and U. W. Heinz, Phys.Lett. B658, 279 (2008), arXiv:0709.0742.

[120] H. Song and U. W. Heinz, Phys.Rev. C77, 064901 (2008), arXiv:0712.3715

[121] S. Bass et al., Prog.Part.Nucl.Phys. 41, 255 (1998), arXiv:nucl-th/9803035

[122] M. Bleicher et al., J.Phys. G25, 1859 (1999), arXiv:hep-ph/9909407.

[123] Y. Aoki, Z. Fodor, S. Katz, and K. Szabo, Phys.Lett. B643, 46 (2006), arXiv:hep-lat/0609068

[124] Y. Aoki et al., JHEP 0906, 088 (2009), arXiv:0903.4155.

[125] S. Borsanyi, Nucl.Phys. A904-905, 270c (2013), arXiv:1210.6901

[126] C. Shen, U. Heinz, P. Huovinen, and H. Song, Phys.Rev. C82, 054904 (2010), arXiv:1010.1856

[127] ALICE, B. B. Abelev et al., (2014), arXiv:1405.4632.

[128] X.-N. Wang and M. Gyulassy, Phys.Rev.Lett. 68, 1480 (1992).

[129] PHENIX Collaboration, K. Adcox et al., Phys.Rev.Lett. 88, 022301 (2002), arXiv:nucl-ex/0109003.

[130] M. Gyulassy, P. Levai, and I. Vitev, Nucl.Phys. B594, 371 (2001), arXiv:nucl-th/0006010.

[131] A. Buzzatti and M. Gyulassy, Phys.Rev.Lett. 108, 022301 (2012), arXiv:1106.3061.

[132] X.-F. Chen, T. Hirano, E. Wang, X.-N. Wang, and H. Zhang, Phys.Rev. C84, 034902 (2011), arXiv:1102.5614

[133] A. Majumder and C. Shen, Phys.Rev.Lett. 109, 202301 (2012), arXiv:1103.0809.

[134] B. Schenke, C. Gale, and S. Jeon, Phys.Rev. C80, 054913 (2009), arXiv:0909.2037.

[135] G.-Y. Qin et al., Phys.Rev.Lett. 100, 072301 (2008), arXiv:0710.0605. 
[136] T. Renk, Phys.Rev. C88, 014905 (2013), arXiv:1302.3710

[137] X.-N. Wang, Phys.Lett. B595, 165 (2004), arXiv:nucl-th/0305010.

[138] H. Zhang, J. Owens, E. Wang, and X.-N. Wang, Phys.Rev.Lett. 98, 212301 (2007), arXiv:nucl-th/0701045.

[139] H. Zhang, J. Owens, E. Wang, and X.-N. Wang, Phys.Rev.Lett. 103, 032302 (2009), arXiv:0902.4000.

[140] S. Albino, B. Kniehl, and G. Kramer, Nucl.Phys. B803, 42 (2008), arXiv:0803.2768.

[141] W.-t. Deng and X.-N. Wang, Phys.Rev. C81, 024902 (2010), arXiv:0910.3403.

[142] X.-F. Chen, C. Greiner, E. Wang, X.-N. Wang, and Z. Xu, Phys.Rev. C81, 064908 (2010), arXiv:1002.1165.

[143] J. Casalderrey-Solana and X.-N. Wang, Phys.Rev. C77, 024902 (2008), arXiv:0705.1352.

[144] L. Pang, Q. Wang, and X.-N. Wang, Phys.Rev. C89, 064910 (2014), arXiv:1309.6735

[145] N.-B. Chang, W.-T. Deng, and X.-N. Wang, Phys.Rev. C89, 034911 (2014), arXiv:1401.5109.

[146] PHENIX Collaboration, A. Adare et al., Phys.Rev.Lett. 101, 232301 (2008), arXiv:0801.4020

[147] PHENIX Collaboration, A. Adare et al., Phys.Rev. C87, 034911 (2013), arXiv:1208.2254.

[148] ALICE Collaboration, B. Abelev et al., Phys.Lett. B720, 52 (2013), arXiv:1208.2711

[149] CMS Collaboration, S. Chatrchyan et al., Eur.Phys.J. C72, 1945 (2012), arXiv:1202.2554.

[150] N. Kidonakis and J. Owens, Phys.Rev. D63, 054019 (2001), arXiv:hep-ph/0007268.

[151] B. Harris and J. Owens, Phys.Rev. D65, 094032 (2002), arXiv:hep-ph/0102128

[152] G.-Y. Qin, J. Ruppert, C. Gale, S. Jeon, and G. D. Moore, Phys.Rev. C80, 054909 (2009), arXiv:0906.3280.

[153] P. B. Arnold, G. D. Moore, and L. G. Yaffe, JHEP 0111, 057 (2001), arXiv:hep-ph/0109064.

[154] P. Arnold, G. D. Moore, and L. G. Yaffe, JHEP 06, 030 (2002), arXiv:hep-ph/0204343.

[155] S. Jeon and G. D. Moore, Phys. Rev. C71, 034901 (2005), arXiv:hep-ph/0309332

[156] I. Vitev, S. Wicks, and B.-W. Zhang, JHEP 0811, 093 (2008), arXiv:0810.2807.

[157] I. Vitev and B.-W. Zhang, Phys.Rev.Lett. 104, 132001 (2010), arXiv:0910.1090

[158] W. Dai, I. Vitev, and B.-W. Zhang, Phys.Rev.Lett. 110, 142001 (2013), arXiv:1207.5177

[159] M. Cacciari, G. P. Salam, and G. Soyez, Eur.Phys.J. C72, 1896 (2012), arXiv:1111.6097.

[160] J. Gao et al., Comput.Phys.Commun. 184, 1626 (2013), arXiv:1207.0513.

[161] K. Eskola, H. Paukkunen, and C. Salgado, JHEP 0904, 065 (2009), arXiv:0902.4154.

[162] I. Lokhtin and A. Snigirev, Eur.Phys.J. C45, 211 (2006), arXiv:hep-ph/0506189.

[163] ATLAS Collaboration, G. Aad et al., (2014), arXiv:1411.2357.

[164] S. Aiola, Nuclear Physics A 931, 382 (2014).

[165] H.-n. Li, Z. Li, and C.-P. Yuan, Phys.Rev.Lett. 107, 152001 (2011), arXiv:1107.4535

[166] H.-n. Li, Z. Li, and C.-P. Yuan, Phys.Rev. D87, 074025 (2013), arXiv:1206.1344.

[167] Y. Mao, Nuclear Physics A 932, 88 (2014).

[168] S. Cao and S. A. Bass, Phys.Rev. C84, 064902 (2011), arXiv:1108.5101,

[169] S. Cao, G.-Y. Qin, and S. A. Bass, Phys.Rev. C88, 044907 (2013), arXiv:1308.0617.

[170] X.-f. Guo and X.-N. Wang, Phys.Rev.Lett. 85, 3591 (2000), arXiv:hep-ph/0005044

[171] A. Majumder, Phys.Rev. D85, 014023 (2012), arXiv:0912.2987

[172] B.-W. Zhang, E. Wang, and X.-N. Wang, Phys.Rev.Lett. 93, 072301 (2004), arXiv:nucl-th/0309040.

[173] CTEQ Collaboration, H. Lai et al., Eur.Phys.J. C12, 375 (2000), arXiv:hep-ph/9903282

[174] Y. Oh, C. M. Ko, S. H. Lee, and S. Yasui, Phys.Rev. C79, 044905 (2009), arXiv:0901.1382.

[175] T. Sjostrand, S. Mrenna, and P. Z. Skands, JHEP 0605, 026 (2006), arXiv:hep-ph/0603175

[176] M. He, R. J. Fries, and R. Rapp, Phys. Rev. C86, 014903 (2012), arXiv:1106.6006.

[177] M. He, R. J. Fries, and R. Rapp, Phys. Rev. Lett. 110, 112301 (2013), arXiv:1204.4442

[178] M. He, R. J. Fries, and R. Rapp, Phys. Rev. C91, 024904 (2015), arXiv:1409.4539.

[179] M. He, R. J. Fries, and R. Rapp, Phys. Lett. B735, 445 (2014), arXiv:1401.3817.

[180] F. Riek and R. Rapp, Phys. Rev. C82, 035201 (2010), arXiv:1005.0769.

[181] K. Huggins and R. Rapp, Nucl. Phys. A896, 24 (2012), arXiv:1206.6537

[182] P. Petreczky and K. Petrov, Phys. Rev. D70, 054503 (2004), arXiv:hep-lat/0405009.

[183] O. Kaczmarek, PoS CPOD07, 043 (2007), arXiv:0710.0498,

[184] F. Riek and R. Rapp, New J. Phys. 13, 045007 (2011), arXiv:1012.0019.

[185] L. Ravagli and R. Rapp, Phys. Lett. B655, 126 (2007), arXiv:0705.0021.

[186] M. He, R. J. Fries, and R. Rapp, Phys. Lett. B701, 445 (2011), arXiv:1103.6279

[187] P. F. Kolb and U. W. Heinz, (2003), arXiv:nucl-th/0305084

[188] T. Matsui and H. Satz, Phys.Lett. B178, 416 (1986).

[189] PHENIX Collaboration, A. Adare et al., Phys.Rev.Lett. 98, 232301 (2007), arXiv:nucl-ex/0611020.

[190] ALICE Collaboration, L. V. Palomo, J.Phys.Conf.Ser. 509, 012111 (2014).

[191] P. Braun-Munzinger and J. Stachel, Phys.Lett. B490, 196 (2000), arXiv:nucl-th/0007059

[192] R. Thews and M. Mangano, Phys.Rev. C73, 014904 (2006), arXiv:nucl-th/0505055.

[193] L. Grandchamp and R. Rapp, Nucl.Phys. A709, 415 (2002), arXiv:hep-ph/0205305

[194] X.-l. Zhu, P.-f. Zhuang, and N. Xu, Phys.Lett. B607, 107 (2005), arXiv:nucl-th/0411093

[195] L. Yan, P. Zhuang, and N. Xu, Phys.Rev.Lett. 97, 232301 (2006), arXiv:nucl-th/0608010

[196] Y.-p. Liu, Z. Qu, N. Xu, and P.-f. Zhuang, Phys.Lett. B678, 72 (2009), arXiv:0901.2757.

[197] K. Zhou, N. Xu, Z. Xu, and P. Zhuang, Phys.Rev. C89, 054911 (2014), arXiv:1401.5845

[198] M. E. Peskin, Nucl.Phys. B156, 365 (1979).

[199] G. Bhanot and M. E. Peskin, Nucl.Phys. B156, 391 (1979). 
[200] H. Satz, J.Phys. G32, R25 (2006), arXiv:hep-ph/0512217.

[201] B. Chen, K. Zhou, and P. Zhuang, Phys.Rev. C86, 034906 (2012), arXiv:1202.3523.

[202] K. Eskola, V. Kolhinen, and C. Salgado, Eur.Phys.J. C9, 61 (1999), arXiv:hep-ph/9807297.

[203] S. Gavin and M. Gyulassy, Phys.Lett. B214, 241 (1988).

[204] U. W. Heinz, H. Song, and A. K. Chaudhuri, Phys.Rev. C73, 034904 (2006), arXiv:nucl-th/0510014.

[205] T. Sjostrand et al., Comput.Phys.Commun. 135, 238 (2001), arXiv:hep-ph/0010017

[206] R. Rapp, G. Chanfray, and J. Wambach, Nucl. Phys. A617, 472 (1997), arXiv:hep-ph/9702210.

[207] M. Urban, M. Buballa, R. Rapp, and J. Wambach, Nucl. Phys. A641, 433 (1998), arXiv:nucl-th/9806030.

[208] R. Rapp and J. Wambach, Eur. Phys. J. A6, 415 (1999), arXiv:hep-ph/9907502.

[209] R. Rapp and J. Wambach, Adv. Nucl. Phys. 25, 1 (2000), arXiv:hep-ph/9909229.

[210] E. Braaten, R. D. Pisarski, and T.-C. Yuan, Phys. Rev. Lett. 64, 2242 (1990).

[211] R. Rapp, Adv.High Energy Phys. 2013, 148253 (2013), arXiv:1304.2309.

[212] H. van Hees and R. Rapp, Nucl. Phys. A806, 339 (2008), arXiv:0711.3444.

[213] S. Turbide, R. Rapp, and C. Gale, Phys. Rev. C69, 014903 (2004), arXiv:hep-ph/0308085

[214] W. Liu and R. Rapp, Nucl. Phys. A796, 101 (2007), arXiv:nucl-th/0604031.

[215] P. B. Arnold, G. D. Moore, and L. G. Yaffe, JHEP 12, 009 (2001), arXiv:hep-ph/0111107

[216] H. van Hees, M. He, and R. Rapp, Nucl. Phys. A933, 256 (2015), arXiv:1404.2846

[217] PHENIX, A. Adare et al., Phys. Rev. Lett. 109, 122302 (2012), arXiv:1105.4126.

[218] ALICE, D. Lohner, J.Phys.Conf.Ser. 446, 012028 (2013), arXiv:1212.3995.

[219] R. Rapp and H. van Hees, (2014), arXiv:1411.4612.

[220] C. Shen et al., (2014), arXiv:1409.8164

[221] M. Alvioli, H. J. Drescher, and M. Strikman, Phys. Lett. B680, 225 (2009), arXiv:0905.2670.

[222] CMS, V. Khachatryan et al., JHEP 01, 079 (2011), arXiv:1011.5531.

[223] Z. Qiu, C. Shen, and U. Heinz, Phys. Lett. B707, 151 (2012), arXiv:1110.3033

[224] C. Shen, U. Heinz, P. Huovinen, and H. Song, Phys. Rev. C84, 044903 (2011), arXiv:1105.3226.

[225] M. Heffernan, P. Hohler, and R. Rapp, Phys. Rev. C91, 027902 (2015), arXiv:1411.7012.

[226] C. Shen, J.-F. Paquet, U. Heinz, and C. Gale, Phys. Rev. C91, 014908 (2015), arXiv:1410.3404.

[227] M. Dion et al., Phys. Rev. C84, 064901 (2011), arXiv:1109.4405.

[228] C. Shen et al., Nucl. Phys. A931, 675 (2014), arXiv:1407.8533.

[229] ALICE, M. Wilde, Nucl. Phys. A904-905, 573c (2013), arXiv:1210.5958.

[230] C. Shen, U. W. Heinz, J.-F. Paquet, and C. Gale, Phys. Rev. C89, 044910 (2014), arXiv:1308.2440 